\title{
Construction of a blow-up solution for the Complex Ginzburg-Landau equation in some critical case
}

\author{
Nejla Nouaili, \\ Nejla.Nouaili@dauphine.fr \\ CEREMADE, Université Paris Dauphine, Paris Sciences et Lettres. \\ Hatem Zaag, \\ Hatem.Zaag@univ-paris13.fr \\ Université Paris 13, Sorbonne Paris Cité, \\ LAGA, CNRS (UMR 7539), F-93430, Villetaneuse, France.
}

September 19, 2018

\begin{abstract}
We construct a solution for the Complex Ginzburg-Landau (CGL) equation in some critical case, which blows up in finite time $T$ only at one blow-up point. We also give a sharp description of its profile. The proof relies on the reduction of the problem to a finite dimensional one, and the use of index theory to conclude. The interpretation of the parameters of the finite dimension problem in terms of the blow-up point and time allows to prove the stability of the constructed solution.
\end{abstract}

Mathematical subject classification: $35 \mathrm{~K} 57,35 \mathrm{~K} 40,35 \mathrm{~B} 44$.

Keywords: Blow-up profile, Complex Ginzburg-Landau equation .

\section{Introduction}

We consider the following complex Ginzburg-Landau (CGL) equation

$$
\begin{aligned}
& u_{t}=(1+i \beta) \Delta u+(1+i \delta)|u|^{p-1} u+\nu u \\
& u(., 0)=u_{0} \in L^{\infty}\left(\mathbb{R}^{N}, \mathbb{C}\right)
\end{aligned}
$$

where $\alpha, \beta, \nu \in \mathbb{R}$.

In fact, in this paper we will treat only the case $\beta=0$, which is an important case in the CGL physics literature (see Aranson and Kramer [AK02]) and which of course can be seen as a complex valued semi-linear heat equation with no variational structure. However, in order to connect our work both to the physics and the math literature, we will consider the case $\beta \in \mathbb{R}$ in the introduction.

This equation, most often considered with a cubic nonlinearity $(p=3)$, has a long history in physics (see Aranson and Kramer [AK02]). The Complex Ginzburg-Landau 
(CGL) equation is one of the most-studied equations in physics, it describes a lot of phenomena including nonlinear waves, second-order phase transitions, and superconductivity. We note that the Ginzburg-Landau equation can be used to describe the evolution of amplitudes of unstable modes for any process exhibiting a Hopf bifurcation (see for example Section VI-C, page 37 and Section VII, page 40 from [AK02 and the references cited therein). The equation can be considered as a general normal form for a large class of bifurcations and nonlinear wave phenomena in continuous media systems. More generally, the Complex Ginzburg-Landau (CGL) equation is used to describe synchronization and collective oscillation in complex media.

The study of blow-up, collapse or chaotic solutions of equation (11) appears in many works; in the description of an unstable plane Poiseuille flow, see Stewartson and Stuart [SS71, Hocking, Stewartson, Stuart and Brown [HSSB72] or in the context of binary mixtures Kolodner and al, [KBS88], KSAL95, where the authors describe an extensive series of experiments on traveling-wave convection in an ethanol/water mixture, and they observe collapse solution that appear experimentally.

For our purpose, we consider CGL independently from any particular physical context and investigate it as a mathematical model in partial differential equations with $p>1$.

We note also that the interest on the study of singular solutions in CGL comes also from the analogies with the three-dimensional Navier-Stokes. The two equations have the same scaling properties and the same energy identity (for more details see the work of Plecháč and Šverák [PŠ01]; the authors in this work give some evidence for the existence of a radial solution which blow up in a self-similar way). Their argument is based on matching a numerical solution in an inner region with an analytical solution in an outer region. In the same direction we can also cite the work of Rottschäfer [Rot08] and [Rot13].

The Cauchy problem for equation (11) can be solved in a variety of spaces using the semigroup theory as in the case of the heat equation (see [Caz03, GV96, GV97]).

We say that $u(t)$ blows up or collapse in finite time $T<\infty$, if $u(t)$ exists for all $t \in[0, T)$ and $\lim _{t \rightarrow T}\|u(t)\|_{L^{\infty}}=+\infty$. In that case, $T$ is called the blow-up time of the solution. A point $x_{0} \in \mathbb{R}^{N}$ is said to be a blow-up point if there is a sequence $\left\{\left(x_{j}, t_{j}\right)\right\}$, such that $x_{j} \rightarrow x_{0}, t_{j} \rightarrow T$ and $\left|u\left(x_{j}, t_{j}\right)\right| \rightarrow \infty$ as $j \rightarrow \infty$. The set of all blow-up points is called the blow-up set.

Let us now introduce the following definition;

Definition 1.1 The exponents $(\beta, \delta)$ are said to be critical (resp. subcritical, resp. supercritical) if $p-\delta^{2}-\beta \delta(p+1)=0$ (resp. $>0$, resp. $\left.<0\right)$.

An extensive literature is devoted to the blow-up profiles for CGL when $\beta=\delta=0$ (which is the nonlinear heat equation), see Velázquez Vel92, Vel93a, Vel93b] and Zaag Zaa02a, Zaa02b, Zaa02c for partial results). In one space dimension, given $a$ a blow-up point, this is the situation:

- either

$$
\sup _{|x-a| \leq K \sqrt{(T-t) \log (T-t)}}\left|(T-t)^{\frac{1}{p-1}} u(x, t)-f\left(\frac{x-a}{\sqrt{(T-t) \mid \log (T-t)}}\right)\right| \rightarrow 0,
$$


- or for some $m \in \mathbb{N}, m \geq 2$, and $C_{m}>0$

$$
\sup _{|x-a|<K(T-t)^{1 / 2 m}}\left|(T-t)^{\frac{1}{p-1}} u(x, t)-f_{m}\left(\frac{C_{m}(x-a)}{(T-t)^{1 / 2 m}}\right)\right| \rightarrow 0,
$$

as $t \rightarrow T$, for any $K>0$, where

$$
\begin{aligned}
& f(z)=\left(p-1+b_{0} z^{2}\right)^{-\frac{1}{p-1}} \text { where } b_{0}=\frac{(p-1)^{2}}{4 p}, \\
& f_{m}(z)=\left(p-1+|z|^{2 m}\right)^{-\frac{1}{p-1}} .
\end{aligned}
$$

If $(\beta, \delta) \neq(0,0)$, the situation is completely understood in the subcritical case by Zaag Zaa98] $(\beta=0)$ and Masmoudi and Zaag [MZ08] $(\delta \neq 0)$. More precisely, if

$$
p-\delta^{2}-\beta \delta(p+1)>0,
$$

then, the authors construct a solution of equation (1), which blows up in finite time $T>0$ only at the origin such that for all $t \in[0, T)$,

$$
\left\|(T-t)^{\frac{1+i \delta}{p-1}}|\log (T-t)|^{-i \mu} u(x, t)-\left(p-1+\frac{b_{\text {sub }}|x|^{2}}{(T-t)|\log (T-t)|}\right)^{-\frac{1+i \delta}{p-1}}\right\|_{L^{\infty}} \leq \frac{C_{0}}{1+\sqrt{|\log (T-t)|}}
$$

where

$$
b_{\text {sub }}=\frac{(p-1)^{2}}{4\left(p-\delta^{2}-\beta \delta(1+p)\right)}>0 \text { and } \mu=-\frac{2 b_{\text {sub }} \beta}{(p-1)^{2}}\left(1+\delta^{2}\right) .
$$

Note that this result was previously obtained formally by Hocking and Stewartson [HS72] $(p=3)$ and mentioned later in Popp and al [PSKK98].

When $p=3$, many works has been devoted to the blow-up profile for the CGL in the subcritical case (see Definition 1.1). We cite the works of Hocking and Stewartson [HS72] and Popp and al and the references cited therein [PSKK98].

In the critical and supercritical cases, few results are known about chaotic solutions for the equation. We note that Hocking and Stewartson [HS72] and Popp and al [PSKK98] proved, formally, the existence of at least two self-similar blow-up solutions, one of them is the same solution constructed by Masmoudi and Zaag in the subcritical case (5). The second kind of blow-up is formally described in [HS72] and PSKK98] and approved by numerical results.

Let us now give the formal result, when $p=3$, given by Popp and al PSKK98] (equations (44) and (64)) in the critical case $\left(3-4 \delta \beta-\delta^{2}=0\right)$ : the authors obtained

$$
(T-t)^{\frac{1+i \delta}{p-1}} u(x, t) \sim e^{i \psi(t)}\left(2+\frac{b_{p}|x|^{2}}{(T-t)|\log (T-t)|^{\frac{1}{2}}}\right)^{-\frac{1+i \delta}{2}} .
$$

where

$$
b_{p}=2\left(\sqrt{\frac{3}{2 \delta^{2}}\left(\delta^{2}+5\right)\left(\delta^{2}+1\right)\left(15-\delta^{2}\right)}\right)^{-1} .
$$

and $\psi(t)$ is given by equation (40) in PSKK98]. We can clearly see, that this profile exist only for $\delta^{2}<15$. 
Remark 1.2 We will see later, in Section [, that we obtain the same result with another formal approach.

In this paper, we justify the formal result of Popp and al [PSKK98], in the case $p=3$, and construct a solution $u(x, t)$ of (1) in the critical case $\left(\beta=0\right.$ and $\left.\delta^{2}=p\right)$ that blows up in some finite time $T$, in the sense that

$$
\lim _{t \rightarrow T}\|u(., t)\|_{L^{\infty}}=+\infty .
$$

More precisely, this is our result:

Theorem 1 (Blow-up profiles for equation (11) Consider $\beta=0, \delta^{2}=p$, then equation (1) has a solution $u(x, t)$, which blows up in finite time $T$, only at the origin. Moreover: (i) for all $t \in[0, T)$,

$$
\left\|(T-t)^{\frac{1+i \delta}{p-1}}|\log (T-t)|^{-i \mu} u(x, t)-\varphi_{0}\left(\frac{x}{\sqrt{(T-t)}|\log (T-t)|^{1 / 4}}\right)\right\|_{L^{\infty}\left(\mathbb{R}^{N}\right)} \leq \frac{C_{0}}{1+|\log (T-t)|^{\frac{1}{4}}}
$$

where

$$
\begin{gathered}
\varphi_{0}(z)=\left(p-1+b z^{2}\right)^{-\frac{1+i \delta}{p-1}}, \\
b=\frac{(p-1)^{2}}{8 \sqrt{p(p+1)}}, \mu=\frac{\delta}{8 p} .
\end{gathered}
$$

(ii) For all $x \neq 0, u(x, t) \rightarrow u^{*}(x) \in C^{2}\left(\mathbb{R}^{N} \backslash\{0\}\right)$ and

$$
\left.u^{*}(x) \sim|2 \log | x\right|^{i \mu}\left[\frac{b|x|^{2}}{\sqrt{2|\log | x||}}\right]^{-\frac{1+i \delta}{p-1}} \text { as } x \rightarrow 0 .
$$

Remark 1.3 We will consider CGL, given by (1), only when $\nu=0$. The case $\nu \neq 0$ can be done as in [EZ11]. In fact, when $\nu \neq 0$, exponentially small terms will be added to our estimate in self-similar variable (see (13) below), and that will be absorbed in our error terms, since our trap $\mathcal{V}_{A}(s)$ defined in Definition 4.3 is given in polynomial scales.

Remark 1.4 The derivation of the blow-up profile (10) can be understood through a formal analysis, using the matching asymptotic expansions (see Section 2 below). This method was used by Galaktionov, Herrero and Velázquez [VGH91] to derive all the possible behaviors of the blow-up solution given by (2|3) in the heat equation $(\beta=\delta=0)$. This formal method was used recently by Tayachi an Zaag [TZ15] in the case of a nonlinear heat equation with a critical power nonlinear gradient term.

However, we would like to emphasize on the fact that our formal analysis is far from being a simple adaptation of this work. We will see in Section 2 that we need much more effort to obtain the profile, this is due to the criticality of the problem $\left(p=\delta^{2}\right)$.

Remark 1.5 The exhibited profile (10) is new in two respects: 
- The scaling law in the critical case is $\sqrt{(T-t)|\log (T-t)|^{\frac{1}{2}}}$ instead of the laws of subcritical case, $\sqrt{(T-t)|\log (T-t)|}$.

- The profile function: $\varphi_{0}(z)=\left(p-1+b|z|^{2}\right)^{-\frac{1+i \delta}{p-1}}$ is different from the profile of the subcritical case, namely $f(z)=\left(p-1+b_{\text {sub }}|z|^{2}\right)^{-\frac{1+i \delta}{p-1}}$, in the sense that $b \neq b_{\text {sub }}$ (see (6) and (11)).

Remark 1.6 In the subcritical case $p-\delta^{2}-\beta \delta(p+1)>0\left(p-\delta^{2}>0\right.$, when $\left.\beta=0\right)$, the final profile of the CGL is given by

$$
u^{*}(x) \sim|2 \log | x||^{i \mu}\left[\frac{b}{2} \frac{|x|^{2}}{|\log | x||}\right]^{-\frac{1+i \delta}{p-1}} \text { as } x \rightarrow 0
$$

with

$$
b=\frac{(p-1)^{2}}{4\left(p-\delta^{2}-\beta \delta(p+1)\right)} \text { and } \mu=-\frac{2 b \beta}{(p-1)^{2}}\left(1+\delta^{2}\right) .
$$

In the critical case $\beta=0, \delta^{2}=p$, the final profile is given by (12).

Remark 1.7 We strongly believe that our construction will not work for all $(\beta, \delta)$ satisfying the critical condition. This is due to the formal study given by Popp and al [PSKK98] in the cubic case. We can see from the definition (8) that $b_{p}$ can be defined only if $\delta^{2}<15$.

As a consequence of our techniques, we show the stability of the constructed solution with respect to perturbations in initial data. More precisely, we have the following result.

Theorem 2 (Stability of the solution constructed in Theorem $10(\beta=0)$ ) Let us denote by $\hat{u}(x, t)$ the solution constructed in Theorem 1 and by $\hat{T}$ its blow-up time. Then, there exists a neighborhood $\mathcal{V}_{0}$ of $\hat{u}(x, 0)$ in $L^{\infty}$ such that for any $u_{0} \in \mathcal{V}_{0}$, equation (1) has a unique solution $u(x, t)$ with initial data $u_{0}$, and $u(x, t)$ blows up in finite time $T\left(u_{0}\right)$ at one single blow-up point $a\left(u_{0}\right)$. Moreover estimate (9) is satisfied by $u(x-a, t)$ and

$$
T\left(u_{0}\right) \rightarrow \hat{T}, \quad a\left(u_{0}\right) \rightarrow 0 \text { as } u_{0} \rightarrow \hat{u}_{0} \text { in } L^{\infty}\left(\mathbb{R}^{N}, \mathbb{C}\right) .
$$

Remark 1.8 We will not give the proof of Theorem 0 because the stability result follows from the reduction to a finite dimensional case as in [MZ97] (see Theorem 2 and its proof in Section 4) and [MZ08] (see Theorem 2 and its proof in Section 6) with the same argument. Hence, we only prove the existence result (Theorem 1) and kindly refer the reader to [MZ97] and [MZ08] for the proof of the stability.

Let us give an idea of the method used to prove the results. We construct the blowup solution with the profile in Theorem 1, by following the method of [MZ97, [BK94], though we are far from a simple adaptation, since we are studying the critical problem, which make the technical details harder to elaborate. This kind of methods has been applied for various nonlinear evolution equations. For hyperbolic equations, it has been successfully used for the construction of multi-solitons for semilinear wave equation in one 
space dimension (see [CZ13]). For parabolic equations, it has been used in [MZ08] and [Zaa01] for the Complex Ginzburg Landau (CGL) equation with no gradient structure, the critical harmonic heat flow in RS13, the two dimensional Keller-Segel equation in [RS14] and the nonlinear heat equation involving nonlinear gradient term in [EZ11] and [TZ15]. Recently, this method has been applied for a non variational parabolic system in [NZ15b] for a logarithmically perturbed nonlinear equation in [NZ15a].

Unlike in subcritical case [MZ08] and [Zaa01]. the criticality of the problem induces substantial changes in the blow-up profile as pointed out in the comments following Theorem 1. Accordingly, its control requires special arguments .So, working in the framework of [MZ97] and [MZ08, some crucial modifications are needed. In particular, we have overcome the following challenges:

- The prescribed profile is not known and not obvious to obtain. See Section 2 for a formal approach to justify such a profile.

- The profile is different from the profile in [MZ97] and [MZ08], therefore new estimates are needed.

- In order to handle the new scaling, we introduce a new shrinking set to trap the solution. See Definition 4.3. Finding such set is not trivial, in particular in the critical case, where we need much more details in the expansions of the rest term see Appendix C.

Then, following [MZ97, the proof is divided in two steps. First, we reduce the problem to a finite dimensional case. Second, we solve the finite time dimensional problem and conclude by contradiction using index theory. The proof is performed in the framework of the similarity variables defined below in (13). We linearize the self-similar solution around the profile $\varphi_{0}$ and we obtain $q$ (see (36) below). Our goal is to guaratee that $q(s)$ belongs to some set $\mathcal{V}_{A}(s)$ (introduced in Definition 4.3), which shrinks to 0 as $s \rightarrow+\infty$. The proof relies on two arguments:

- The linearized equation gives two positives mode; $q_{0}$ and $q_{1}$, one zero modes $\left(q_{2}\right)$ and an infinite dimensional negative part. The negative part is easily controlled by the effect of the heat kernel. The control of the zero mode is quite delicate (see Part 2: Proof of Proposition 4.10, page 37). Consequently, the control of $q$ is reduced to the control of its positive modes.

- The control of the positive modes $q_{0}$ and $q_{1}$ is handled thanks to a topological argument based on index theory (see the argument at page 18).

The organization of the rest of this paper is as follows. In Section 2 , we explain formally how we obtain the profile. In Section 3, we give a formulation of the problem in order to justify the formal argument. Section 4 is divided in two subsections. In Subsection 4.1 we give the proof of the existence of the profile assuming technical details. In particular, we construct a shrinking set and give an example of initial data giving rise to prescribed blowup profile. Subsection 4.2 is devoted to the proof of technical results which are needed in the proof of existence. Finally, in Section 5 , we give the proof of Theorem 1 .

Acknowledgment: The authors would like to thank the referees for their valuable suggestions which (we hope) made our paper much clearer and reader friendly. 


\section{Formal approach}

We consider CGL, given by (1), when $\nu=0$, as we mentioned before in Remark 1.3. The aim of this section is to explain formally how we derive the behavior given in Theorem 1. In particular, how to obtain the profile $\varphi_{0}$ in (10), the parameter $b$ in (11). Consider an arbitrary $T$ and the self-similar transformation of (11)

$$
w(y, s)=(T-t)^{\frac{1+i \delta}{p-1}} u(x, t), \quad y=\frac{x}{\sqrt{T-t}}, \quad s=-\log (T-t) .
$$

If $u(x, t)$ satisfies (10) for all $(x, t) \in \mathbb{R}^{N} \times[0, T)$, then $w(y, s)$ satisfies for all $(x, t) \in$ $\mathbb{R}^{N} \times[-\log T,+\infty)$ the following equation

$$
\frac{\partial w}{\partial s}=\Delta w-\frac{1}{2} y \cdot \nabla w-\frac{1+i \delta}{p-1} w+(1+i \delta)|w|^{p-1} w,
$$

for all $(y, s) \in \mathbb{R}^{N} \times[-\log T,+\infty)$. Thus constructing a solution $u(x, t)$ for the equation (11) that blows up at $T<\infty$ like $(T-t)^{-\frac{1}{p-1}}$ reduces to constructing a global solution $w(y, s)$ for equation (14) such that

$$
0<\varepsilon \leq \lim _{s \rightarrow \infty}\|w(s)\|_{L^{\infty}\left(\mathbb{R}^{N}\right)} \leq \frac{1}{\varepsilon} .
$$

A first idea to construct a blow-up solution for (11), would be to find a stationary solution of (14), yielding a self-similar solution for (1). It happens, that in the subcritical case, the second author together with Masmoudi were able in [MZ08 to construct such a solution. Of course, the construction of [MZ08] is in the same spirit as the approach used for the heat equation in [MZ97] and [BK94], in the sense that the authors in [MZ08] cut in space the solution on similarity variables. However, the profile they get is different from the case of the standard heat equation, in the sense that they obtain blow-up for the mode and also for the phase, with a different constant in the profile (see $b_{\text {sub }}([6)$ ).

In the critical case $\left(\beta=0, p=\delta^{2}\right)$, there is no self-similar solution apart from the trivial constant solution $w \equiv \kappa$ of (14) (see Proposition 2.1 in [NZ10] or simply multiply equation (14) by $w \rho$, where

$$
\rho(y)=\frac{e^{-\frac{|y|^{2}}{4}}}{(4 \pi)^{N / 2}}, y \in \mathbb{R}^{N}
$$

and integrate in space).

\section{$2.1 \quad$ Inner expansion.}

Following the approach of Bricmont and Kupiainen [BK94] and Masmoudi and Zaag [MZ08, we may look for a solution $w$ to (13) such that $v \rightarrow 0$ as $s \rightarrow \infty$, if $v$ is defined by the following ansatz;

$$
w(y, s)=e^{i \mu \log s}(v(y, s)+\kappa) \text { where } \kappa=(p-1)^{-\frac{1}{p-1}} .
$$

Using (13), we see that $v$ should satisfy the equation 


$$
\frac{\partial v}{\partial s}=\tilde{\mathcal{L}} v+F(v)-i \frac{\mu}{s} \kappa-i \frac{\mu}{s} v
$$

with

$$
\begin{gathered}
\tilde{\mathcal{L}} v=\Delta v-\frac{1}{2} y \cdot \nabla v+(1+i \delta) v_{1}, \text { where } v_{1}=\operatorname{Re}(v), \\
F(v)=(1+i \delta)\left(|v+\kappa|^{p-1}(v+\kappa)-\kappa^{p}-\frac{v}{p-1}-v_{1}\right) .
\end{gathered}
$$

Let us recall some properties of $\tilde{\mathcal{L}}$. The operator $\tilde{\mathcal{L}}$ is a $\mathbb{R}$-linear operator defined on $L_{\rho}^{2}\left(\mathbb{R}^{N}, \mathbb{C}\right)$ where

$$
L_{\rho}^{2}\left(\mathbb{R}^{N}, \mathbb{C}\right)=\left\{\left.f \in L_{l o c}^{2}\left(\mathbb{R}^{N}, \mathbb{C}\right)\left|\int_{\mathbb{R}^{N}}\right| f(y)\right|^{2} \rho(y) d y<\infty\right\}
$$

and $\rho(y)$ is defined by (16).

The spectrum of $\tilde{\mathcal{L}}$ is explicitly given by

$$
\left\{1-\frac{m}{2} \mid m \in \mathbb{N}\right\} .
$$

For $N=1$, the eigenfunctions are given by

$$
\left\{(1+i \delta) h_{m}(y), i h_{m}(y) \mid m \in \mathbb{N}\right\},
$$

where $h_{m}$ are rescaled versions of Hermite polynomials given by:

$$
h_{m}(y)=\sum_{n=0}^{\left[\frac{m}{2}\right]} \frac{m !}{n !(m-2 n) !}(-1)^{n} y^{m-2 n} .
$$

Note that we have

$$
\begin{cases}\tilde{\mathcal{L}}\left((1+i \delta) h_{m}\right) & =\left(1-\frac{m}{2}\right)(1+i \delta) h_{m} \\ \tilde{\mathcal{L}}\left(i h_{m}\right) & =-\frac{m}{2} i h_{m}\end{cases}
$$

Since the family $h_{m}$ is a basis of the Hilbert space $L_{\rho}^{2}\left(R^{N}, R\right)$, we see that each $r \in L_{\rho}^{2}$ can be uniquely decomposed as

$$
r(y)=(1+i \delta) R_{1}(y)+i R_{2}(y)=(1+i \delta)\left(\sum_{m=0}^{+\infty} R_{1 m} h_{m}\right)+i\left(\sum_{m=0}^{+\infty} R_{2 m} h_{m}\right)
$$

where

$$
\begin{aligned}
& R_{1}(y)=\operatorname{Re}(r(y)), R_{2}(y)=\operatorname{Im}(r(y))-\delta \operatorname{Re}(r(y)), \\
& \text { and for } i=1,2, \quad R_{i m}(y)=\int_{\mathbb{R}} R_{i}(y) \frac{h_{m}(y)}{\left\|h_{m}(y)\right\|_{L_{\rho}^{2}}^{2}} \rho(y) d y .
\end{aligned}
$$

Note that $\tilde{\mathcal{L}}$ is not $\mathbb{C}$-linear. In addition it is not self-adjoint, but fortunately it can be written as follows in a diagonal form. Indeed, using the notation (23), we see that

$$
\tilde{\mathcal{L}} r=(1+i \delta) \mathcal{L} R_{1}+i(\mathcal{L}-\mathcal{I}) R_{2}
$$


where $\mathcal{L} h=\Delta h-\frac{1}{2} y \cdot \nabla h+h$.

In compliance with the spectral properties of $\tilde{\mathcal{L}}$, we may look for a solution expanded as follows:

$$
v(y, s)=(1+i \delta) \sum_{m \in \mathbb{N}} \bar{v}_{m}(s) h_{m}(y)+i \sum_{m \in \mathbb{N}} \hat{v}_{m}(s) h_{m}(y) .
$$

Since the eigenfunctions for $m \geq 3$ correspond to negative eigenvalues of $\tilde{\mathcal{L}}$, assuming $v$ is even, we may look for a solution $v(y, s)$ for equation (18) in the form:

$$
v(y, s)=(1+i \delta)\left(\bar{v}_{0} h_{0}+\bar{v}_{2} h_{2}\right)+i \hat{v}_{0} h_{0}(y) .
$$

Then projecting equation (18), we derive the following ODE system

$$
\left\{\begin{aligned}
\bar{v}_{0}^{\prime}= & \bar{v}_{0}+\frac{\mu \delta}{s} \bar{v}_{0}+\frac{\mu}{s} \hat{v}_{0}+\frac{1}{2 \kappa} \hat{v}_{0}^{2}-\frac{(p+1) p}{3 \kappa^{2}} \bar{v}_{0}^{3}-\frac{(p+1) \delta}{\kappa^{2}} \bar{v}_{0}^{2} \hat{v}_{0}-\frac{(p+1)}{\kappa^{2}} \bar{v}_{0} \hat{v}_{0}^{2}-8 \frac{(p+1)}{\kappa^{2}} \bar{v}_{0} \bar{v}_{2}^{2} \\
& -\frac{\delta}{2 \kappa^{2}} \hat{v}_{0}^{3}-8 \frac{(p+1) \delta}{\kappa^{2}} \hat{v}_{0} \bar{v}_{2}^{2}-\frac{64}{3} \frac{(p+1) p}{\kappa^{2}} \bar{v}_{2}^{3}+R_{1}, \\
\bar{v}_{2}^{\prime}= & \frac{\mu \delta}{s} \bar{v}_{2}-40 \frac{(p+1) p}{\kappa^{2}} \bar{v}_{2}^{3}-8 \frac{(p+1) p}{\kappa^{2}} \bar{v}_{2}^{2} \bar{v}_{0}-8 \frac{(p+1) \delta}{\kappa^{2}} \bar{v}_{2}^{2} \hat{v}_{0} \\
& -\frac{(p+1) p}{\kappa^{2}} \bar{v}_{2} \bar{v}_{0}^{2}-\frac{(p+1)}{\kappa^{2}} \bar{v}_{2} \hat{v}_{0}^{2}-2 \frac{(p+1) \delta}{\kappa^{2}} \bar{v}_{2} \bar{v}_{0} \hat{v}_{0}+R_{1} \\
\hat{v}_{0}^{\prime}= & -\frac{\mu \kappa}{s}-\frac{\mu(1+p)}{s} \bar{v}_{0}-\frac{\mu \delta}{s} \hat{v}_{0}+\frac{1+p}{\kappa} \hat{v}_{0} \bar{v}_{0}+\frac{(1+p) \delta}{\kappa} \bar{v}_{0}^{2}+\frac{8(1+p) \delta}{\kappa} \bar{v}_{2}^{2} \\
& -\frac{\delta(p+1)^{2}}{\kappa^{2}} \bar{v}_{0}^{3}+\frac{(2 p-1)(p+1)}{\kappa^{2}} \bar{v}_{0}^{2} \hat{v}_{0}+\frac{3 \delta(p+1)}{2 \kappa^{2}} \bar{v}_{0} \hat{v}_{0}^{2}+\frac{(p+1)}{2 \kappa^{2}} \hat{v}_{0}^{3}, \\
& -24 \frac{\delta(p+1)^{2}}{\kappa^{2}} \bar{v}_{0} \bar{v}_{2}^{2}+8 \frac{(2 p-1)(p+1)}{\kappa^{2}} \hat{v}_{0} \bar{v}_{2}^{2}-64 \frac{\delta(p+1)^{2}}{\kappa^{2}} \bar{v}_{2}^{3}+R_{1},
\end{aligned}\right.
$$

where $R_{1}=O\left(\left|\bar{v}_{0}\right|^{4}+\left|\hat{v}_{0}\right|^{4}+\left|\bar{v}_{2}\right|^{4}\right)$.

Looking for a solution satisfying the ansatz

$$
\bar{v}_{2} \sim \frac{\alpha}{\sqrt{s}}, \quad \hat{v}_{0} \sim \frac{\beta}{\sqrt{s}} \text { and } \bar{v}_{0}=O\left(\frac{1}{s}\right), \text { where } \alpha, \beta \in \mathbb{R},
$$

we obtain by the last equation of (26)

$$
\mu=\frac{8 \delta(p+1)}{\kappa^{2}} \alpha^{2}
$$

Note that the ansatz (27) seems to be compatible with our ODE (26). Of course, in order to determine $\alpha$ and $\beta$, we need to refine (27) adding smaller terms, and the process never stops. For that reason, we abandon that procedure, and proceed in a different way in order to determine $\alpha$.

Indeed, in order to determine the value of $\alpha$, one may use the formal approach of the physicists in [PSKK98], Section 3.3, page 93, and see that when we have

$$
p=3, \quad \alpha=-\frac{\sqrt{2}}{16 \sqrt{3}}
$$


If $p \neq 3$, we are able to successfully carry on that method and find the value of $\alpha$, and also $\mu$ by (28):

$$
\alpha=-\frac{\kappa}{8 \sqrt{p(p+1)}}, \quad \mu=\frac{\delta}{8 p} .
$$

In fact, this calculation for $p \neq 3$ is easier than in PSKK98 since we already know the good scaling from (27). However, in order to keep the paper in a reasonable length, we choose to not include that calculation here and we refer the interested reader to the physicists paper [PSKK98].

In fact, we should keep in mind that we have a better justification of the value of $\alpha$ in (29): that value is precisely the one that makes our rigorous proof work in the next section! Indeed, we see from Corollary 4.5 below that this particular value of $\alpha$, makes some projections small and allows us to conclude.

Therefore, since we took $w(y, s)=e^{i \mu \log s}(v(y, s)+\kappa)$ from (17), it follows from (25), (27) and (29) that

$$
\begin{aligned}
w(y, s) & =e^{i \mu \log s}\left[\kappa-\frac{\kappa}{8 \sqrt{p(p+1)}} \frac{y^{2}-2}{\sqrt{s}}(1+i \delta)+i \frac{\beta}{\sqrt{s}}+o\left(\frac{1}{\sqrt{s}}\right)\right], \\
& =e^{i \mu \log s+i \frac{\beta}{\kappa \sqrt{s}}}\left[\kappa-\frac{\kappa}{8 \sqrt{p(p+1)}} \frac{y^{2}-2}{\sqrt{s}}(1+i \delta)+o\left(\frac{1}{\sqrt{s}}\right)\right],
\end{aligned}
$$

in $L_{\rho}^{2}\left(\mathbb{R}^{N}\right)$, and also uniformly on compact sets by standard parabolic regularity.

We would like to note that the term $i \beta /(\kappa \sqrt{s})$ in the phase is compatible with our rigorous proof. Indeed, the modulation parameter $\theta(s)$ we introduce in our formulation of the problem below in (35) will be shown to satisfy $|\theta(s)| \leq C / \sqrt{s}$ below in (122) (note that we may take $\theta_{0}=0$ in (122) from the rotation invariance of equation (14)). Accordingly, we could have chosen to make a modulation technique already in this formal step, but as one may easily check, there is a one-to-one relation between the modulation parameter and the coordinate $\hat{v}_{0}(s)$ and he result and well as the calculations will be completely the same.

\subsection{Outer expansion}

From (30), we see that the variable

$$
z=\frac{y}{s^{1 / 4}}
$$

as given by (9), is perhaps the relevant variable for blow-up. Unfortunately, (30) provides no shape, since it is valid only on compact sets (note that $z \rightarrow 0$ as $s \rightarrow \infty$ in this case). In order to see some shape, we may need to go further in space, to the "outer region", namely when $z \neq 0$. In view of (30), we may try to find an expression of $w$ of the form

$$
w(y, s)=e^{i \mu \log s+i \frac{\beta}{\kappa \sqrt{s}}}\left[\varphi_{0}(z)+(1+i \delta) \frac{\kappa}{4 \sqrt{p(p+1)}} \frac{1}{\sqrt{s}}+o\left(\frac{1}{\sqrt{s}}\right)\right] \text { with } z=\frac{y}{s^{1 / 4}} .
$$


Plugging this ansatz in equation (18), keeping only the main order, we end-up with the following equation on $\varphi_{0}$ :

$$
-\frac{1}{2} z \cdot \nabla \varphi_{0}(z)-\frac{1+i \delta}{p-1} \varphi_{0}(z)+(1+i \delta)\left|\varphi_{0}(z)\right|^{p-1} \varphi_{0}(z)=0, \quad z=\frac{y}{s^{1 / 4}} .
$$

Recalling that our aim is to find $w$ a solution of (18) such that $w \rightarrow \kappa$ as $s \rightarrow \infty$ (in $L_{\rho}^{2}$, hence uniformly on every compact set), we derive from (31) ( with $y=z=0$ ) the natural condition

$$
\varphi_{0}(0)=\kappa .
$$

Therefore, integrating equation (32), we see that

$$
\varphi_{0}(z)=\left(p-1+b z^{2}\right)^{-\frac{1+i \delta}{p-1}},
$$

for some $b \in \mathbb{R}$. Recalling also that we want a solution $w \in L^{\infty}\left(\mathbb{R}^{N}\right)$, we see that $b \geq 0$ and for a nontrivial solution, we should have

$$
b>0 .
$$

- Thus, we have just obtained from (31) that

$w(y, s)=e^{i \mu \log s+i \frac{\beta}{\kappa \sqrt{s}}}\left[\left(p-1+b z^{2}\right)^{-\frac{1+i \delta}{p-1}}+(1+i \delta) \frac{\kappa}{4 \sqrt{p(p+1)}} \frac{1}{\sqrt{s}}+o\left(\frac{1}{\sqrt{s}}\right)\right]$ with $z=\frac{y}{s^{1 / 4}}$.

We should understand this expansion to be valid at least on compact sets in $z$, that is for $|y|<R s^{\frac{1}{4}}$, for any $R>0$.

\subsection{Matching asymptotics}

Since (33) holds for $|y|<R s^{\frac{1}{4}}$, for any $R>0$, it holds also uniformly on compact sets, leading to the following expansion for $y$ bounded:

$$
w(y, s)=e^{i \mu \log s+i \frac{\beta}{\kappa \sqrt{s}}}\left[\kappa-(1+i \delta) \frac{\kappa b}{(p-1)^{2}} \frac{y^{2}}{s^{\frac{1}{2}}}+(1+i \delta) \frac{a}{s^{\frac{1}{2}}}+o\left(\frac{1}{\sqrt{s}}\right)\right]
$$

Comparing with (30), we find the following values of $a$ and $b$ :

$$
b=\frac{(p-1)^{2}}{8 \sqrt{p(p+1)}}, \quad a=\frac{\kappa}{4 \sqrt{p(p+1)}} .
$$

In conclusion, we see that we have just derived the following profile for $w(y, s)$ :

$$
w(y, s) \sim e^{i \mu \log s+i \frac{\beta}{\kappa \sqrt{s}}} \varphi(y, s)
$$

with

$$
\begin{aligned}
& \varphi(y, s)=\varphi_{0}\left(\frac{y}{s^{1 / 4}}\right)+(1+i \delta) \frac{a}{s^{1 / 2}} \equiv \kappa^{-i \delta}\left(p-1+b \frac{|y|^{2}}{s^{1 / 2}}\right)^{-\frac{1+i \delta}{p-1}}+(1+i \delta) \frac{a}{s^{1 / 2}} \\
& b=\frac{(p-1)^{2}}{8 \sqrt{p(p+1)}}, \quad a=\frac{\kappa}{4 \sqrt{p(p+1)}}, \quad \mu=\frac{\delta}{8 p} \text { and } \kappa=(p-1)^{-\frac{1}{p-1}} .
\end{aligned}
$$




\section{Formulation of the problem}

We recall that we consider CGL, given by (1), when $\nu=0$, as we mentioned before in Remark 1.3 .

The preceding calculation is purely formal. However, the formal expansion provides us with the profile of the function $\left(w(y, s)=e^{i \mu \log s}\left(\varphi_{0}\left(\frac{y}{s^{1 / 4}}\right)+\ldots\right)\right)$. Our idea is to linearize equation (14) around that profile and prove that the linearized equation as well as the nonlinear equation have a solution that goes to zero as $s \rightarrow \infty$. Let us introduce $q(y, s)$ and $\theta(s)$ such that

$$
w(y, s)=e^{i(\mu \log s+\theta(s))}(\varphi(y, s)+q(y, s)),
$$

where $\varphi(y, s)=\varphi_{0}\left(\frac{y}{s^{1 / 4}}\right)+(1+i \delta) \frac{a}{s^{1 / 2}} \equiv \kappa^{-i \delta}\left(p-1+b \frac{|y|^{2}}{s^{1 / 2}}\right)^{-\frac{1+i \delta}{p-1}}+(1+i \delta) \frac{a}{s^{1 / 2}}$

$$
b=\frac{(p-1)^{2}}{8 \sqrt{p(p+1)}}, \quad a=\frac{\kappa}{4 \sqrt{p(p+1)}}, \quad \mu=\frac{\delta}{8 p} \text { and } \kappa=(p-1)^{-\frac{1}{p-1}} .
$$

In order to guarantee the uniqueness of the couple $(q, \theta)$ an additional constraint is needed, see (58) below; we will choose $\theta(s)$ such that we kill one the neutral modes of the linearized operator.

Note that $\varphi_{0}(z)=w_{0}(z)$ has been exhibited in the formal approach and satisfies the following equation

$$
-\frac{1}{2} z \nabla w_{0}-\frac{1+i \delta}{p-1} w_{0}+(1+i \delta)\left|w_{0}\right|^{p-1} w_{0}=0,
$$

which makes $\varphi(y, s)$ an approximate solution of (14). If $w$ satisfies equation (14), then $q$ satisfies the following equation

$$
\frac{\partial q}{\partial s}=\mathcal{L}_{0} q-\frac{(1+i \delta)}{p-1} q+L\left(q, \theta^{\prime}, y, s\right)+R^{*}\left(\theta^{\prime}, y, s\right)
$$

where

$$
\begin{array}{ll}
\mathcal{L}_{0} q & =\Delta q-\frac{1}{2} y \cdot \nabla q \\
L\left(q, \theta^{\prime}, y, s\right) & =(1+i \delta)\left\{|\varphi+q|^{p-1}(\varphi+q)-|\varphi|^{p-1} \varphi-i\left(\frac{\mu}{s}+\theta^{\prime}(s)\right) q\right\} \\
R^{*}\left(\theta^{\prime}, y, s\right) & =R(y, s)-i\left(\frac{\mu}{s}+\theta^{\prime}(s)\right) \varphi \\
R(y, s) & =-\frac{\partial \varphi}{\partial s}+\Delta \varphi-\frac{1}{2} y \cdot \nabla \varphi-\frac{(1+i \delta)}{p-1} \varphi+(1+i \delta)|\varphi|^{p-1} \varphi
\end{array}
$$

Our aim is to find a $\theta \in C^{1}([-\log T, \infty), \mathbb{R}$ such that equation (42) has a solution $q(y, s)$ defined for all $(y, s) \in \mathbb{R}^{N} \times[-\log T, \infty)$ such that

$$
\|q(s)\|_{L^{\infty}} \rightarrow 0 \text { as } s \rightarrow \infty .
$$

From (37), one sees that the variable $z=\frac{y}{s^{1 / 4}}$ plays a fundamental role. Thus, we will consider the dynamics for $|z|>K$ and $|z|<2 K$ separately for some $K>0$ to be fixed large. 


\subsection{The outer region where $|y|>K s^{1 / 4}$}

Let us consider a non-increasing cut-off function $\chi_{0} \in C^{\infty}\left(\mathbb{R}^{+},[0,1]\right)$ such that $\chi_{0}(\xi)=1$ for $\xi<1$ and $\chi_{0}(\xi)=0$ for $\xi>2$ and introduce

$$
\chi(y, s)=\chi_{0}\left(\frac{|y|}{K s^{1 / 4}}\right),
$$

where $K$ will be fixed large. Let us define

$$
q_{e}(y, s)=e^{\frac{i \delta}{p-1} s} q(y, s)(1-\chi(y, s))
$$

$q_{e}$ is the part of $q(y, s)$ for $|y|>K s^{1 / 4}$. As we will explain in subsection (4.3.3), the linear operator of the equation satisfied by $q_{e}$ is negative, which makes it easy to control $\left\|q_{e}(s)\right\|_{L^{\infty}}$. This is not the case for the part of $q(y, s)$ for $|y|<2 K s^{1 / 4}$, where the linear operator has two positive eigenvalues, a zero eigenvalue in addition to infinitely many negative ones. Therefore, we have to expand $q$ with respect to these eigenvalues in order to control $\|q(s)\|_{L^{\infty}\left(|y|<2 K s^{1 / 4}\right)}$. This requires more work than for $q_{e}$. The following subsection is dedicated to that purpose. From now on, $K$ will be fixed constant which is chosen such that $\left\|\varphi\left(s^{\prime}\right)\right\|_{L^{\infty}\left(|y|>K s^{\prime 1 / 4}\right)}$ is small enough, namely $\left\|\varphi_{0}(z)\right\|_{L^{\infty}(|z|>K)}^{p-1} \leq \frac{1}{C(p-1)}$ (see subsection (4.3.3) below, for more details).

\subsection{The inner region where $|y|<2 K s^{1 / 4}$}

If we linearize the term $L\left(q, \theta^{\prime}, y, s\right)$ in equation (38), then we can write (38) as

$$
\frac{\partial q}{\partial s}=\tilde{\mathcal{L}} q-i\left(\frac{\mu}{s}+\theta^{\prime}(s)\right) q+V_{1} q+V_{2} \bar{q}+B(q, y, s)+R^{*}\left(\theta^{\prime}, y, s\right)
$$

where

$$
\begin{array}{ll}
\tilde{\mathcal{L}} q & =\Delta q-\frac{1}{2} y \cdot \nabla q+(1+i \delta) \operatorname{Re} q, \\
V_{1}(y, s) & =(1+i \delta) \frac{p+1}{2}\left(|\varphi|^{p-1}-\frac{1}{p-1}\right), \quad V_{2}(y, s)=(1+i \delta) \frac{p-1}{2}\left(|\varphi|^{p-3} \varphi^{2}-\frac{1}{p-1}\right), \\
B(q, y, s) & =(1+i \delta)\left(|\varphi+q|^{p-1}(\varphi+q)-|\varphi|^{p-1} \varphi-|\varphi|^{p-1} q-\frac{p-1}{2}|\varphi|^{p-3} \varphi(\varphi \bar{q}+\bar{\varphi} q)\right), \\
R^{*}\left(\theta^{\prime}, y, s\right) & =R(y, s)-i\left(\frac{\mu}{s}+\theta^{\prime}(s)\right) \varphi \\
R(y, s) & =-\frac{\partial \varphi}{\partial s}+\Delta \varphi-\frac{1}{2} y \cdot \nabla \varphi-\frac{(1+i \delta)}{p-1} \varphi+(1+i \delta)|\varphi|^{p-1} \varphi
\end{array}
$$

Note that the term $B(q, y, s)$ is built to be quadratic in the inner region $|y| \leq K s^{1 / 4}$. Indeed, we have for all $K \geq 1$ and $s \geq 1$,

$$
\sup _{|y| \leq 2 K s^{1 / 4}}|B(q, y, s)| \leq C(K)|q|^{2}
$$

Note also that $R(y, s)$ measures the defect of $\varphi(y, s)$ from being an exact solution of (14). However, since $\varphi(y, s)$ is an approximate solution of (14) , one easily derives the fact that

$$
\|R(s)\|_{L^{\infty}} \leq \frac{C}{\sqrt{s}} .
$$


Therefore, if $\theta^{\prime}(s)$ goes to zero as $s \rightarrow \infty$, we expect the term $R^{*}\left(\theta^{\prime}, y, s\right)$ to be small, since (42) and (45) yield

$$
\left|R^{*}\left(\theta^{\prime}, y, s\right)\right| \leq \frac{C}{\sqrt{s}}+\left|\theta^{\prime}(s)\right|
$$

Therefore, since we would like to make $q$ go to zero as $s \rightarrow \infty$, the dynamics of equation (42) are influenced by the asymptotic limit of its linear term,

$$
\tilde{\mathcal{L}}+V_{1} q+V_{2} \bar{q}
$$

as $s \rightarrow \infty$. In the sense of distribution (see the definition of $V_{1}$ and $V_{2}$ (42) and $\varphi(34)$ ) this limit is $\tilde{\mathcal{L}}$.

\subsection{Decomposition of $q$}

For the sake of controlling $q$ in the region $|y|<2 K s^{1 / 4}$, by the spectral properties of $\tilde{\mathcal{L}}$ (22), we will expand the unknown function $q$ with respect to the family $h_{n}$ and then, with respect to the families

$$
\tilde{h}_{n}=(1+i \delta) h_{n} \text { and } \hat{h}_{n}=i h_{n}
$$

We start by writing

$$
q(y, s)=\sum_{n \leq M} Q_{n}(s) h_{n}(y)+q_{-}(y, s),
$$

where $h_{n}$ is the eigenfunctions of $\mathcal{L}$ defined in (21) $, Q_{n}(s) \in \mathbb{C}, q_{-}$satisfy

$$
\begin{gathered}
Q_{n}(s)=\frac{\int q h_{n} \rho}{\int h_{n}^{2} \rho}, \\
\int q_{-}(y, s) h_{n}(y) \rho(y) d y=0 \text { for all } n \leq M,
\end{gathered}
$$

and $M$ is a fixed even integer satisfying

$$
M \geq 4\left(\sqrt{1+\delta^{2}}+1+2 \max _{i=1,2, y \in \mathbb{R}, s \geq 1}\left|V_{i}(y, s)\right|\right),
$$

with $V_{i=1,2}$ defined in (43).

The function $q_{-}(y, s)$ can be seen as the projection of $q(y, s)$ onto the spectrum of $\tilde{\mathcal{L}}$, which is smaller than $(1-M) / 2$. We will call it the infinite dimensional part of $q$ and we will denote it $q_{-}=P_{-, M}(q)$. We also introduce $P_{+, M}=I d-P_{-, M}$. Notice that $P_{-, M}$ and $P_{+, M}$ are projections. In the sequel, we will denote $P_{-}=P_{-, M}$ and $P_{+}=P_{+, M}$. The complementary part $q_{+}=q-q_{-}$will be called the finite dimensional part of $q$. We will expand it as follows

$$
q_{+}(y, s)=\sum_{n \leq M} Q_{n}(s) h_{n}(y)=\sum_{n \leq M} \tilde{q}_{n}(s) \tilde{h}_{n}(y)+\hat{q}_{n}(s) \hat{h}_{n}(y)
$$

where $\tilde{q}_{n}, \hat{q}_{n} \in \mathbb{R}, \tilde{h}_{n}=(1+i \delta) h_{n}$ and $\hat{h}_{n}=i h_{n}$. Finally, we notice that for all $s$, we have

$$
\int q_{-}(y, s) q_{+}(y, s) \rho(y) d y=0
$$


Our purpose is to project (42) in order to write an equation for $\tilde{q}_{n}$ and $\hat{q}_{n}$. Note that

$$
\tilde{P}_{n}(q)=\tilde{q}_{n}(s)=\operatorname{Re} Q_{n}(s), \hat{P}_{n}(q)=\hat{q}_{n}(s)=\operatorname{Im} Q_{n}(s)-\delta \operatorname{Re} Q_{n}(s) .
$$

We conclude from (48) and (51), that

$$
q(y, s)=\left(\sum_{n \leq M} \tilde{q}_{n}(s) \tilde{h}_{n}(y)+\hat{q}_{n}(s) \hat{h}_{n}(y)\right)+q_{-}(y, s),
$$

where $\tilde{h}_{n}$ and $\hat{h}_{n}$ are given by (47). we should keep in mind that this decomposition is unique.

\section{Existence}

In this section, we prove the existence of a solution $q(s), \theta(s)$ of problem (38)-(58) such that

$$
\lim _{s \rightarrow \infty}\|q\|_{L^{\infty}}=0, \text { and }\left|\theta^{\prime}(s)\right| \leq C \frac{A^{5}}{s^{3 / 2}} \text { for all } s \in[-\log T,+\infty) .
$$

Hereafter, we denote by $C$ a generic positive constant, depending only on $p$ and $K$ introduced in (40), itself depending on $p$. In particular, $C$ does not depend on $A$ and $s_{0}$, the constants that will appear shortly and throughout the paper and need to be adjusted for the proof.

We proceed in two subsections. In the first, we give the proof assuming the technicals details. In the second subsection we give the proof of the technicals details.

\subsection{Proof of the existence assuming technical results}

Our construction is built on a careful choice of the initial data of $q$ at a time $s_{0}$. We will choose it in the following form:

Definition 4.1 (Choice of initial data) Let us define, for $A \geq 1, s_{0}=-\log T>1$ and $d_{0}, d_{1} \in \mathbb{R}$, the function

$$
\begin{aligned}
\psi_{s_{0}, d_{0}, d_{1}}(y) & =\left[\frac{A}{s_{0}^{3 / 2}}(1+i \delta)\left(d_{0} h_{0}(y)+d_{1} h_{1}(y)\right)+i d_{2}\right] \chi\left(2 y, s_{0}\right) \\
\text { where } s_{0} & =-\log T
\end{aligned}
$$

where $h_{i}, i=0,1,2$ are defined by (21), $\chi$ is defined by (40) and $d_{2}=d_{2}\left(d_{0}, d_{1}\right)$ will be fixed later in (i) of Proposition 4.5.

Remark 4.2 Let us recall that we will modulate the parameter $\theta$ to kill one of the neutral modes, see equation (58) below. It is natural that this condition must be satisfied for the initial data at $s=s_{0}$. Thus, it is necessary that we choose $d_{2}$ to satisfy condition (58), see (59) below. 
The solution of equation (42) will be denoted by $q_{s_{0}, d_{0}, d_{1}}$ or $q$ when there is no ambiguity. We will show that if $A$ is fixed large enough, then, $s_{0}$ is fixed large enough depending on $A$, we can fix the parameters $\left(d_{0}, d_{1}\right) \in[-2,2]^{2}$, so that the solution $v_{s_{0}, d_{0}, d_{1}} \rightarrow 0$ as $s \rightarrow \infty$ in $L^{\infty}(\mathbb{R})$, that is (54) holds. Owing to the decomposition given in (53), it is enough to control the solution in a shrinking set defined as follows

Definition 4.3 (A set shrinking to zero) For all $K>1, A \geq 1$ and $s \geq e$, we define $\mathcal{V}_{A}(s)$ as the set of all $r \in L^{\infty}(\mathbb{R})$ such that

$$
\begin{array}{ll}
\left\|r_{e}\right\|_{L^{\infty}(\mathbb{R})} \leq \frac{A^{M+2}}{s^{\frac{1}{4}}}, & \left\|\frac{r_{-}(y)}{1+|y|^{M+1}}\right\|_{L^{\infty}(\mathbb{R})} \leq \frac{A^{M+1}}{s^{\frac{M+2}{4}}}, \\
\left|\hat{r}_{j}\right|,\left|\tilde{r}_{j}\right| \leq \frac{A^{j^{4}}}{s^{\frac{j+1}{4}}} \text { for all } 3 \leq j \leq M, & \left|\tilde{r}_{0}\right|,\left|\tilde{r}_{1}\right| \leq \frac{A}{s^{\frac{3}{2}}}, \\
\left|\hat{r}_{0}\right| \leq \frac{1}{s^{\frac{3}{2}}},\left|\hat{r}_{1}\right| \leq \frac{A^{4}}{s^{\frac{3}{2}}}, & \left|\tilde{r}_{2}\right| \leq \frac{A^{5}}{s}, \quad\left|\hat{r}_{2}\right| \leq \frac{A^{3}}{s},
\end{array}
$$

Since $A \geq 1$, the sets $\mathcal{V}_{A}(s)$ are increasing (for fixed $s$ ) with respect to $A$ in the sense of inclusions.

We also show the following property of elements of $\mathcal{V}_{A}(s)$ :

For all $A \geq 1$, there exists $s_{01}(A) \geq 1$, such that for all $s \geq s_{01}$ and $r \in \mathcal{V}(A)$, we have

$$
\|r\|_{L^{\infty}}(\mathbb{R}) \leq C(K) \frac{A^{M+2}}{s^{\frac{1}{4}}}
$$

where $C$ is a positive constant (see Claim 4.8 below for the proof).

By (57), if a solution $q$ stays in $\mathcal{V}(A)$ for $s \geq s_{01}$, then it converges to 0 in $L^{\infty}(\mathbb{R})$.

So far, the phase $\theta(s)$ introduced in (35) is arbitrary, in fact as we will show below in Proposition 4.6. We can use a modulation technique to choose $\theta(s)$ in such a way that we impose the condition

$$
\hat{P}_{0}(q(s))=0,
$$

which allows us to kill the neutral direction of the operator $\tilde{\mathcal{L}}$ defined in (42). Reasonably, our aim is then reduced to the following proposition:

Proposition 4.4 (Existence of a solution trapped in $\mathcal{V}_{A}(s)$ ) There exists $A_{2} \geq 1$ such that for $A \geq A_{2}$ there exists $s_{02}(A)$ such that for all $s_{0} \geq s_{02}(A)$, there exists $\left(d_{0}, d_{1}\right)$ such that if $q$ is the solution of (42)-(58), with initial data given by (55) and (59), then $v \in \mathcal{V}_{A}(s)$, for all $s \geq s_{0}$.

This proposition gives the stronger convergence to 0 in $L^{\infty}(\mathbb{R})$ thanks to (57).

Let us first be sure that we can choose the initial data such that it starts in $\mathcal{V}_{A}\left(s_{0}\right)$. In other words, we will define a set where where will be selected the good parameters $\left(d_{0}, d_{1}\right)$ that will give the conclusion of Proposition 4.4. More precisely, we have the following result:

Proposition 4.5 (Properties of initial data) For each $A \geq 1$, there exists $s_{03}(A)>1$ such that for all $s_{0} \geq s_{03}$ :

(i) $\hat{P}_{0}\left(i \chi\left(2 y, s_{0}\right)\right) \neq 0$ and the parameter $d_{2}\left(s_{0}, d_{0}, d_{1}\right)$ given by

$$
d_{2}\left(s_{0}, d_{0}, d_{1}\right)=-\frac{A}{s_{0}^{3 / 2}} \frac{d_{0} \hat{P}_{0}\left((1+i \delta) \chi\left(2 y, s_{0}\right)\right)+d_{1} \hat{P}_{0}\left((1+i \delta) y \chi\left(2 y, s_{0}\right)\right)}{\hat{P}_{0}\left(i \chi\left(2 y, s_{0}\right)\right)}
$$


is well defined, where $\chi$ defined in (40).

(ii) If $\psi$ is given by (55) and (59) with $d_{2}$ defined by (59). Then, there exists a quadrilateral $\mathcal{D}_{s_{0}} \subset[-2,2]^{2}$ such that the mapping $\left(d_{0}, d_{1}\right) \rightarrow\left(\tilde{\psi}_{0}, \tilde{\psi}_{1}\right)$ (where $\psi$ stands for $\left.\psi_{s_{0}, d_{0}, d_{1}}\right)$ is linear, one to one from $\mathcal{D}_{s_{0}}$ onto $\left[-\frac{A}{s_{0}^{3 / 2}}, \frac{A}{s_{0}^{3 / 2}}\right]^{2}$. Moreover it is of degree 1 on the boundary. (iii) For all $\left(d_{0}, d_{1}\right) \in \mathcal{D}_{s_{0}}, \psi_{e} \equiv 0, \hat{\psi}_{0}=0,\left|\tilde{\psi}_{i}\right|+\left|\hat{\psi}_{i}\right| \leq C A e^{-\gamma s_{0}}$ for some $\gamma>0$, for some $\gamma>0$ and for all $3 \leq i \leq M$ and $1 \leq j \leq M$. Moreover, $\left\|\frac{\psi_{-}(y)}{(1+|y|)^{M+1}}\right\|_{L^{\infty}(\mathbb{R})} \leq C \frac{A}{s_{0}^{\frac{M}{4}+1}}$.

(iv) For all $\left(d_{0}, d_{1}\right) \in \mathcal{D}_{s_{0}}, \psi_{s_{0}, d_{0}, d_{1}} \in \mathcal{V}_{A}\left(s_{0}\right)$ with strict inequalities except for $\left(\tilde{\psi}_{0}, \tilde{\psi}_{1}\right)$.

The proof of previous proposition is postponed to subsection 4.2 .

In the following, we find a local in time solution for equation (42) coupled with the condition (58).

Proposition 4.6 (Local in time solution and modulation for problem (42)-(58) with initial data (55) -(59) ) For all $A \geq 1$, there exists $T_{3}(A) \in(0,1 / e)$ such that for all $T \leq T_{3}$, the following holds:

For all $\left(d_{0}, d_{1}\right) \in D_{T}$, there exists $s_{\max }>s_{0}=-\log T$ such that problem (42)-(158) with initial data at $s=s_{0}$,

$$
\left(q\left(s_{0}\right), \theta\left(s_{0}\right)\right)=\left(\psi_{s_{0}, d_{0}, d_{1}}, 0\right),
$$

where $\psi_{s_{0}, d_{0}, d_{1}}$ is given by (55) and (59), has a unique solution $q(s), \theta(s)$ satisfying $q(s) \in$ $V_{A+1}(s)$ for all $s \in\left[s_{0}, s_{\max }\right)$.

The proof of this proposition will be given later in page 21.

Let us now give the proof of Proposition 4.4.

Proof of Proposition 4.4. Let us consider $A \geq 1, s_{0} \geq s_{03},\left(d_{0}, d_{1}\right) \in \mathcal{D}_{s_{0}}$, where $s_{03}$ is given by Proposition 4.5. From the existence theory (which follows from the Cauchy problem for equation (1)), starting in $\mathcal{V}_{A}\left(s_{0}\right)$ which is in $\mathcal{V}_{A+1}\left(s_{0}\right)$, the solution stays in $\mathcal{V}_{A}(s)$ until some maximal time $s_{*}=s_{*}\left(d_{0}, d_{1}\right)$. Then, either:

- $s_{*}\left(d_{0}, d_{1}\right)=\infty$ for some $\left(d_{0}, d_{1}\right) \in \mathcal{D}_{s_{0}}$, then the proof is complete.

- $s_{*}\left(d_{0}, d_{1}\right)<\infty$, for any $\left(d_{0}, d_{1}\right) \in \mathcal{D}_{s_{0}}$, then we argue by contradiction. By continuity and the definition of $s_{*}$, the solution on $s_{*}$ is in the boundary of $\mathcal{V}_{A}\left(s_{*}\right)$. Then, by definition of $\mathcal{V}_{A}\left(s_{*}\right)$, one at least of the inequalities in that definition is an equality. Owing to the following proposition, this can happen only for the first two components $\tilde{q}_{0}, \tilde{q}_{1}$. Precisely we have the following result

Proposition 4.7 (Control of $q(s)$ by $\left(q_{0}(s), q_{1}(s)\right.$ ) in $\mathcal{V}_{A}(s)$ ). There exists $A_{4} \geq 1$ such that for each $A \geq A_{4}$, there exists $s_{04} \in \mathbb{R}$ such that for all $s_{0} \geq s_{04}$. The following holds:

If $q$ is a solution of (42) with initial data at $s=s_{0}$ given by (55) and (59) with $\left(d_{0}, d_{1}\right) \in$ $\mathcal{D}_{s_{0}}$, and $q(s) \in \mathcal{V}(A)(s)$ for all $s \in\left[s_{0}, s_{1}\right]$, with $q\left(s_{1}\right) \in \partial \mathcal{V}_{A}\left(s_{1}\right)$ for some $s_{1} \geq s_{0}$, then:

(i)(Smallness of the modulation parameter $\theta$ defined in (17)) For all $s \in\left[s_{0}, s_{1}\right]$,

$$
\left|\theta^{\prime}(s)\right| \leq \frac{C A^{5}}{s^{3 / 2}}
$$


(ii) (Reduction to a finite dimensional problem) We have:

$$
\left(\tilde{q}_{0}\left(s_{1}\right), \tilde{q}_{1}\left(s_{1}\right)\right) \in \partial\left(\left[-\frac{A}{s_{1}^{\frac{3}{2}}}, \frac{A}{s_{1}^{\frac{3}{2}}}\right]^{2}\right) .
$$

(iii)(Transverse crossing) There exists $m \in\{0,1\}$ and $\omega \in\{-1,1\}$ such that

$$
\omega \tilde{q}_{m}\left(s_{1}\right)=\frac{A}{s_{1}^{\frac{3}{2}}} \text { and } \omega \frac{d \tilde{q}_{m}}{d s}\left(s_{1}\right)>0 .
$$

Assume the result of the previous proposition, for which the proof is given below in page 21, and continue the proof of Proposition 4.4. Let $A \geq A_{4}$ and $s_{0} \geq s_{04}(A)$. It follows from Proposition 4.7, part (ii) that $\left(q_{0}\left(s_{*}\right), q_{1}\left(s_{*}\right)\right) \in \partial\left(\left[-\frac{A}{s_{*}^{\frac{3}{2}}}, \frac{A}{s_{*}^{\frac{3}{2}}}\right]^{2}\right)$, and the following function

$$
\begin{aligned}
\phi \quad & \mathcal{D}_{s_{0}} \rightarrow \partial\left([-1,1]^{2}\right) \\
& \left(d_{0}, d_{1}\right) \rightarrow \frac{s_{*}^{3 / 2}}{A}\left(q_{0}, q_{1}\right)_{\left(d_{0}, d_{1}\right)}\left(s_{*}\right), \text { with } s_{*}=s_{*}\left(d_{0}, d_{1}\right),
\end{aligned}
$$

is well defined. Then, it follows from Proposition 4.7, part (iii) that $\phi$ is continuous. On the other hand, using Proposition 4.5 (ii)-(iv) together with the fact that $q\left(s_{0}\right)=\psi_{s_{0}, d_{0}, d_{1}}$, we see that when $\left(d_{0}, d_{1}\right)$ is in the boundary of the rectangle $\mathcal{D}_{s_{0}}$, we have strict inequalities for the other components.

Applying the transverse crossing property given by (iii) of Proposition 4.7, we see that $q(s)$ leaves $\mathcal{V}_{A}(s)$ at $s=s_{0}$, hence $s_{*}\left(d_{0}, d_{1}\right)=s_{0}$. Using Proposition 4.5, part (ii), we see that the restriction of $\phi$ to the boundary is of degree 1. A contradiction, then follows from the index theory. Thus there exists a value $\left(d_{0}, d_{1}\right) \in \mathcal{D}_{s_{0}}$ such that for all $s \geq s_{0}$, $q_{s_{0}, d_{0}, d_{1}}(s) \in \mathcal{V}_{A}(s)$. This concludes the proof of Proposition 4.4.

Using (i) of Proposition 4.7, we get the bound on $\theta^{\prime}(s)$. This concludes the proof of (54).

\subsection{Proof of the technical results result}

This section is devoted to the proof of the existence result given by Theorem 1, We proceed in 4 steps, each of them making a separate subsection.

- In the first subsection, we give some properties of the shrinking set $\mathcal{V}_{A}(s)$ defined by (56) and translate our goal of making $q(s)$ go to 0 in $L^{\infty}(\mathbb{R})$ in terms of belonging to $\mathcal{V}_{A}(s)$. We also give the proof of Proposition 4.5.

- In second subsection, we solve the local in time Cauchy problem for equation (42) coupled with some orthogonality condition.

- In the third subsection using the spectral properties of equation (42), we reduce our goal from the control of $q(s)$ (an infinite dimensional variable) in $\mathcal{V}_{A}(s)$ to control its two first components $\left(\tilde{q}_{0}, \tilde{q}_{1}\right)$ a two variables in $\left[-\frac{A}{s^{\frac{3}{2}}}, \frac{A}{s^{\frac{3}{2}}}\right]^{2}$.

- In the fourth subsection, we solve the finite dimensional problem using the index theory and conclude the proof of Theorem 1 . 


\subsubsection{Properties of the shrinking set $\mathcal{V}_{A}(s)$ and preparation of initial data}

In this subsection, we give some properties of the shrinking set defined by (56). Let us first introduce the following claim:

Claim 4.8 (Properties of the shrinking set defined by (56) ) For all $r \in \mathcal{V}_{A}(s)$,

(i) $\|r\|_{L^{\infty}\left(|y|<2 K s^{\frac{1}{4}}\right)} \leq C(K) \frac{A^{M+1}}{s^{\frac{1}{4}}}$ and $\|r\|_{L^{\infty}}(\mathbb{R}) \leq C(K) \frac{A^{M+2}}{s^{\frac{1}{4}}}$.

(ii) for all $y \in \mathbb{R},|r(y)| \leq C \frac{A^{M+1}}{s}\left(1+|y|^{M+1}\right)$.

Proof: Take $r \in \mathcal{V}_{A}(s)$ and $y \in \mathbb{R}$.

(i) If $|y| \geq 2 K s^{\frac{1}{4}}$, then we have from the definition of $r_{e}$ (41), $|r(y)|=\left|r_{e}(y)\right| \leq \frac{A^{M+2}}{s^{\frac{1}{4}}}$.

Now, if $|y|<2 K s^{\frac{1}{4}}$, since we have for all $0 \leq j \leq M,\left|\tilde{r}_{j}\right|+\left|\hat{r}_{j}\right| \leq C \frac{A^{j}}{s^{\frac{j+1}{4}}}$ from (156)) (use the fact that $M \geq 4$ ), we write from (53)

$$
\begin{aligned}
|r(y)| & \leq\left(\sum_{j \leq M}\left|\tilde{h}_{j}\right|\left|\tilde{h}_{j}\right|+\left|\hat{h}_{j}\right|\left|\hat{h}_{j}\right|\right)+\left|r_{-}(y)\right|, \\
& \leq C \sum_{j \leq M} \frac{A^{M+1}}{s^{\frac{j+1}{4}}}(1+|y|)^{j}+\frac{A^{M+1}}{s^{\frac{M+2}{4}}}(1+|y|)^{M+1}, \\
& \leq C \sum_{j \leq M} \frac{A^{M+1}}{s^{\frac{j+1}{4}}}\left(1+K s^{\frac{1}{4}}\right)^{j}+\frac{A^{M+1}}{s^{\frac{M+2}{4}}}\left(1+K s^{\frac{1}{4}}\right)^{M+1} \leq C \frac{(K A)^{M+1}}{s^{\frac{1}{4}}}
\end{aligned}
$$

which gives (i).

(ii) Just use (60) together with the fact that for all $0 \leq j \leq M,\left|\tilde{r}_{j}\right|+\left|\hat{r}_{j}\right| \leq C \frac{A^{M+1}}{s}$ from (56). This ends the proof of Claim 4.8,

Let us now give the proof of Proposition 4.5.

Proof of Proposition 4.5For simplicity, we write $\psi$ instead of $\psi_{s_{0}, d_{0}, d_{1}}$. We note that, from Claim 4.8, (iv) follows from (ii) and (iii) by taking $s_{0}=-\log T$ large enough (that is $T$ is small enough). Thus, we only prove (i), (ii) and (iii). Consider $K \geq 1, A \geq 1$ and $T \leq 1 / e$. Note that $s_{0}=-\log T \geq 1$.

The proof of (i) is a direct consequence of (iii) of the following claim

Claim 4.9 There exists $\gamma=\frac{1}{64}>0$ and $T_{2}<1 / e$ such that for all $K \geq 1$ and $T \leq T_{2}$, if $g$ is given by $(1+i \delta) \chi\left(2 y, s_{0}\right),(1+i \delta) y \chi\left(2 y, s_{0}\right),(1+i \delta) h_{2}(y) \chi\left(2 y, s_{0}\right)$ or $i \chi\left(2 y, s_{0}\right)$, then $\left\|\frac{g_{-}(y)}{1+|y|^{M+1}}\right\|_{L^{\infty}} \leq \frac{C}{s_{0}^{\frac{M}{4}}}$ and all $\hat{g}_{i}, \tilde{g}_{i}$ for $0 \leq i \leq M$ are less than $C e^{-\gamma s_{0}}$. expect:

i) $\left|\tilde{g}_{0}-1\right| \leq C e^{-\gamma s_{0}}$ when $g=(1+i \delta) \chi\left(2 y, s_{0}\right)$.

ii) $\left|\tilde{g}_{1}-1\right| \leq C e^{-\gamma s_{0}}$ when $g=(1+i \delta) y \chi\left(2 y, s_{0}\right)$.

iii) $\left|\hat{g}_{0}-1\right| \leq C e^{-\gamma s_{0}}$ when $g=i \chi\left(2 y, s_{0}\right)$.

Proof: In all cases, we write

$g(y)=p(y)+r(y)$ where $p(y)=(1+i \delta)$ or $(1+i \delta) y$ or $i$ and $r(y)=p(y)\left(\chi\left(2 y, s_{0}\right)-1\right)$.

From the uniqueness of the decomposition (53), we see that $p_{-} \equiv 0$ and al $\hat{p}_{i}, \tilde{p}_{i}$ are zero except 
$\tilde{p}_{0}=1($ when $p(y)=(1+i \delta)), \tilde{p}_{1}=1($ when $p(y)=(1+i \delta) y)$ and $\hat{p}_{0}=1($ when $p(y)=i)$. Concerning the cases $2|y|<K s^{\frac{1}{4}}$ and $2|y|>K s^{\frac{1}{4}}$, we have the definition of $\chi$ (40),

$$
\begin{gathered}
1-\chi(2 y, s) \leq\left(\frac{2|y|}{K s_{0}^{\frac{1}{4}}}\right)^{M-1}, \\
\rho(y)(1-\chi(2 y, s)) \leq \sqrt{\rho(y)} \sqrt{\rho\left(\frac{K}{2} s^{\frac{1}{4}}\right)} \leq C e^{-\frac{K^{2} s_{0}}{64} \sqrt{\rho(y)} .}
\end{gathered}
$$

Therefore, from (53) and (61), we see that

$$
\begin{aligned}
& |r(y)| \leq C\left(1+|y|^{2}\right)\left(\frac{2|y|}{K s_{0}^{\frac{1}{4}}}\right)^{M-1} \leq C \frac{\left(1+|y|^{M+1}\right)}{s_{0}^{\frac{M}{4}}}, \\
& \left|\hat{r}_{j}\right|+\left|\tilde{r}_{j}\right| \leq C e^{-\frac{K^{2} \sqrt{s_{0}}}{64}} \text { for all } j \leq M .
\end{aligned}
$$

Hence, using (62) and (48) and the fact that $\left|r_{j}(y)\right| \leq C(1+|y|)^{M}$, for all $j \leq M$, we get also

$$
\left|r_{-}(y)\right| \leq C \frac{(1+|y|)^{M}}{s_{0}^{\frac{M}{4}}} .
$$

Using (61) and the estimates for $p(y)$ stated below, we conclude the proof of Claim 4.9 and (i) of Proposition 4.5.

(ii) of Proposition 4.5: From (55) and (59), we see that

$$
\left(\begin{array}{c}
\tilde{\psi}_{0} \\
\tilde{\psi}_{1}
\end{array}\right)=G\left(\begin{array}{c}
d_{0} \\
d_{1}
\end{array}\right) \text { where } G=\left(g_{i, j}\right)_{0 \leq i, j \leq 1}
$$

Using Claim 4.9, we see from (55) and (59) that

$$
\left|d_{2}\right| \leq C\left(\left|d_{0}\right|+\left|d_{1}\right|\right) e^{-\gamma s_{0}}
$$

for $T$ small enough. Using again Claim 4.9, We see that $\frac{s_{0}^{\frac{3}{2}}}{A} G \rightarrow I d$ and

as $s_{0} \rightarrow \infty$ (for fixed $K$ and $A$ ), which concludes the proof of (ii) of Proposition 4.5. (iii) of Proposition 4.5. Since $\operatorname{supp}(\psi) \subset B\left(0, K s_{0}^{\frac{1}{4}}\right)$ by (55) and (159), we see that $\psi_{e} \equiv 0$ and that

$$
\begin{aligned}
\hat{\psi}_{0}=\hat{P}_{0}(\psi) & =\frac{A}{s_{0}^{\frac{3}{2}}}\left(d_{0} \hat{P}_{0}\left((1+i \delta) \chi\left(2 y, s_{0}\right)\right)+d_{1} \hat{P}_{0}\left((1+i \delta) y \chi\left(2 y, s_{0}\right)\right)\right) \\
& +d_{2} \hat{P}_{0}\left(i \chi\left(2 y, s_{0}\right)\right),
\end{aligned}
$$

which is zero from the definition of $d_{2}$ (55) and (59). Using the fact that $\left|d_{i, i=0,1}\right| \leq 2$ and the bound on $d_{2}$ by (64), we see that the estimates on $\hat{\psi}_{j}$ and $\tilde{\psi}_{j}$ and $\psi_{-}$in (iii) follows from (55) and (59) and Claim 4.9, This concludes the proof of Proposition 4.5.

In the following we give the proof of Local in time solution for problem (42)-(58). In fact, we impose some orthogonality condition given by (58), killing the one of the zero eigenfunctions of the linearized operator of equation (42). 
Proof of Proposition 4.6: From solution of the local in time Cauchy problem for equation (11) in $L^{\infty}(\mathbb{R})$, there exists $s_{1}>s_{0}$ such that equation (14) with initial data (at $\left.s=s_{0}\right) \varphi\left(y, s_{0}\right)+\psi_{s_{0}, d_{0} d_{1}}(y)$, where $\varphi(y, s)$ is given by (34) has a unique solution $w(s) \in C\left(\left[s_{0}, s_{1}\right), L^{\infty}(\mathbb{R})\right)$. Now, we have to find a unique $(q(s), \theta(s))$ such that

$$
w(y, s)=e^{i(\mu \log s+\theta(s))}(\varphi(y, s)+q(y, s))
$$

and (58) is satisfied. Using (52), we can write (58) as follows

$\hat{P}_{0}(q)=\operatorname{Im}\left(\int q(y, s) \rho(y) d y\right)-\delta \operatorname{Re}\left(\int q(y, s) \rho(y) d y\right)=\operatorname{Im}\left((1-i \delta) \int q(y, s) \rho(y) d y\right)=0$,

or using (65)

$$
F(s, \theta) \equiv \operatorname{Im}\left((1-i \delta) \int\left(e^{-i(\mu \log s+\theta(s))} w(y, s)-\varphi(y, s)\right) \rho(y) d y\right)=0 .
$$

Note that

$$
\frac{\partial F}{\partial \theta}(s, \theta)=-\operatorname{Re}\left((1-i \delta) \int e^{-i(\mu \log s+\theta(s))} w(y, s) \rho(y) d y\right) .
$$

From (iii) in Proposition 4.5, $F\left(s_{0}, 0\right)=P_{0, M}\left(\psi_{s_{0}, d_{0}, d_{1}}\right)=0$ and

$\frac{\partial F}{\partial \theta}\left(s_{0}, 0\right)=-\operatorname{Re}\left((1-i \delta) \int\left(\varphi\left(y, s_{0}\right)+\psi_{s_{0}, d_{0} d_{1}}(y)\right) \rho(y) d y\right)=-\kappa+O\left(\frac{1}{s_{0}^{1 / 4}}\right)$ as $s_{0} \rightarrow \infty$

for fixed $K$ and $A$.

Therefore, if $T$ is small enough in terms of $A$, then $\frac{\partial F}{\partial \theta}\left(s_{0}, 0\right) \neq 0$, and from the implicit function Theorem, there exists $s_{2} \in\left(s_{0}, s_{1}\right)$ and $\theta \in C^{1}\left(\left[s_{0}, s_{2}\right), \mathbb{R}\right)$ such that $F(s, \theta(s))=0$ for all $s \in\left[s_{0}, s_{2}\right)$. Defining $q(s)$ by (65) gives a unique solution of the problem (43)-(58) for all $s \in\left[s_{0}, s_{2}\right)$. Now, since we have from (iv) of Proposition 4.5, $q\left(s_{0}\right) \in V_{A}\left(s_{0}\right) \stackrel{\subset}{\neq} V_{A+1}\left(s_{0}\right)$, there exists $s_{3} \in\left(s_{0}, s_{2}\right)$ such that for all $s \in\left[s_{0}, s_{3}\right)$, $q(s) \in V_{A+1}(s)$. This concludes the proof of Proposition 4.6.

\subsubsection{Reduction to a finite dimensional problem}

In the following we give the proof of Proposition 4.7

The idea of the proof is to project equation (42) on the different components of the decomposition (53). More precisely, we claim that Proposition 4.7 is a consequence of the following

Proposition 4.10 There exists $A_{5} \geq 1$ such that for all $A \geq A_{5}$, there exists $s_{5}(A)$ such that the following holds for all $s_{0} \geq s_{5}$ :

Assuming that for all $s \in\left[\tau, s_{1}\right]$ for some $s_{1} \geq \tau \geq s_{0}, q(s) \in \mathcal{V}_{A}(s)$ and $\hat{q}_{0}(s)=0$, then the following holds for all $s \in\left[\tau, s_{1}\right]$ : 
(i) (Smallness of the modulation parameter):

$$
\left|\theta^{\prime}(s)\right| \leq C \frac{A^{5}}{s^{\frac{3}{2}}}
$$

(ii) (ODE satisfied by the expanding mode): For $m=0$ and 1 , we have

$$
\left|\tilde{q}_{m}^{\prime}-\left(1-\frac{m}{2}\right) \tilde{q}_{m}\right| \leq \frac{C}{s^{\frac{3}{2}}} .
$$

(iii) (ODE satisfied by the null mode):

$$
\left|\tilde{q}_{2}^{\prime}+\frac{2}{s} \tilde{q}_{2}\right| \leq \frac{C A^{3}}{s^{2}}
$$

(iv) (Control of null and negative modes):

$$
\begin{gathered}
\left|\hat{q}_{1}(s)\right| \leq e^{-\frac{(s-\tau)}{2}}\left|\hat{q}_{1}(\tau)\right|+\frac{C A^{5}}{s^{\frac{3}{2}}}, \\
\left|\hat{q}_{2}(s)\right| \leq e^{-(s-\tau)}\left|\hat{q}_{2}(\tau)\right|+\frac{C}{s}, \\
\left|\hat{q}_{j}(s)\right| \leq e^{-j \frac{(s-\tau)}{2}}\left|\hat{q}_{j}(\tau)\right|+\frac{C A^{j-1}}{s^{\frac{j+1}{4}}}, \text { for all } 3 \leq j \leq M, \\
\left|\tilde{q}_{j}(s)\right| \leq e^{-(j-2) \frac{(s-\tau)}{2}}\left|\tilde{q}_{j}(\tau)\right|+\frac{C A^{j-1}}{s^{\frac{j+1}{4}}}, \text { for all } 3 \leq j \leq M, \\
\left\|\frac{q_{-}(y, s)}{1+|y|^{M+1}}\right\|_{L^{\infty}} \leq e^{-\frac{M+1}{4}(s-\tau)}\left\|\frac{q_{-}(\tau)}{1+|y|^{M+1}}\right\|_{L^{\infty}}+C \frac{A^{M}}{s^{\frac{M+2}{4}}}, \\
\left\|q_{e}(y, s)\right\|_{L^{\infty}} \leq e^{-\frac{(s-\tau)}{2(p-1)}}\left\|q_{e}(\tau)\right\|_{L^{\infty}+} \frac{C A^{M+1}}{\tau^{\frac{1}{4}}}(1+s-\tau)
\end{gathered}
$$

The idea of the proof of Proposition 4.10 is to project equations (38) and (42) according to the decomposition (53). However because of the number of parameters and coordinates in (53), the computation become too long. That is why Subsection 4.3 is devoted to the proof of Proposition 4.10.

Remark 4.11 The coefficient in front of $\frac{\tilde{q}_{2}}{s}$ in (iii) of Proposition 4.10 is ' 2 '. In our proof, see page 38 below, that coefficient is the sum of four contributions, which depend on $p$ in a non trivial way. Thus, it may appear miraculous to see the sum of such contributions equal to '2'. The same phenomena occur in the subcritical range of parameters, see [MZ08] and also the heat equation, with a critical gradient term (see [TZ15]). In fact, adopting the approach of Pierre Raphaël and co-authors, one may see that the coefficient ' 2 ' appears in a natural way due to scaling considerations (From a personal communication of Pierre Raphaël). 
Let us now derive Proposition 4.7 from Proposition 4.10.

Proof of Proposition 4.7 assuming Proposition 4.10:

We will take $A_{4} \geq A_{5}$. Hence, we can use the conclusion of Proposition 4.10.

(i) The proof follows from (i) of Proposition 4.10, Indeed by choosing $T_{4}$ small enough, we can make $s_{0}=-\log T$ bigger than $s_{5}(A)$.

(ii) We notice that from Claim 4.8 and the fact that $\hat{q}_{0}(s)=0$, it is enough to prove that for all $s \in\left[s_{0}, s_{1}\right]$,

$$
\begin{gathered}
\left|\tilde{q}_{2}(s)\right|<\frac{A^{5}}{s} . \\
\left\|q_{e}\right\|_{L^{\infty}(\mathbb{R}) \leq \frac{A^{M+2}}{2 s^{\frac{1}{4}}}}, \quad\left\|\frac{q_{-}(y, s)}{1+|y|^{M+1}}\right\|_{L^{\infty}} \leq \frac{A^{M+1}}{2 s^{\frac{M+2}{4}}}, \\
\left|\hat{q}_{j}\right|,\left|\tilde{q}_{j}\right| \leq \frac{A^{j}}{2 s^{\frac{j+1}{4}}} \text { for all } 3 \leq j \leq M, \quad\left|\hat{q}_{1}\right| \leq \frac{A^{4}}{2 s^{\frac{3}{2}}}, \quad\left|\hat{q}_{2}\right| \leq \frac{A^{3}}{2 s} .
\end{gathered}
$$

Let us first prove (66). Arguing by contradiction, we assume that

$$
\tilde{q}_{2}\left(s_{*}\right)=\omega \frac{A^{5}}{s_{*}} \text { and for all } s \in\left[s_{0}, s^{*}\left[, \quad\left|\tilde{q}_{2}(s)\right|<\frac{A^{5}}{s} .\right.\right.
$$

Of course, we can reduce to the case $\omega=1$. Note by (iv) of Proposition 4.5 that $\left|\tilde{q}_{2}\left(s_{0}\right)\right|<\frac{A^{5}}{s_{0}}$, hence $s_{*}>s_{0}$, and the interval $\left[s_{0}, s_{*}\right]$ is not empty.

By minimality, it follows that $\tilde{q}_{2}^{\prime}(s) \geq \frac{\partial}{\partial s}\left(\frac{A^{5}}{s}\right)_{\mid s=s_{*}}$,

$$
\tilde{q}_{2}^{\prime}(s) \geq-\frac{A^{5}}{s_{*}^{2}}
$$

in the one hand, recalling, from (iii) of Proposition 4.10, that

$$
\left|\tilde{q}_{2}^{\prime}+\frac{2}{s} \tilde{q}_{2}\right| \leq \frac{C A^{3}}{s^{2}}
$$

We write

$$
\tilde{q}_{2}^{\prime}\left(s_{*}\right) \leq-2 \frac{\tilde{q}_{2}\left(s_{*}\right)}{s_{*}}+\frac{C A^{3}}{s_{*}^{2}}=\frac{-2 A^{5}+C A^{3}}{s_{*}^{2}},
$$

for $A$ large enough a contradiction follows from (68) and (69). Thus (66) holds.

Now, let us deal with (67). Define $\sigma=\log A$ and take $s_{0} \geq \sigma$ (that is $T \leq e^{-\sigma}=1 / A$ ) so that for all $\tau \geq s_{0}$ and $s \in[\tau, \tau+\sigma]$, we have

$$
\tau \leq s \leq \tau+\sigma \leq \tau+s_{0} \leq 2 \tau \text { hence } \frac{1}{2 \tau} \leq \frac{1}{s} \leq \frac{1}{\tau} \leq \frac{2}{s} .
$$

We consider two cases in the proof.

Case 1: $s \leq s_{0}+\sigma$. 
Note that (70) holds with $\tau=s_{0}$. Using (iv) of Proposition 4.10 and estimate (iii) of Proposition 4.5 on the initial data $q\left(., s_{0}\right)$ (where we use (70) with $\tau=s_{0}$ ), we write

$$
\begin{aligned}
& \left|\hat{q}_{1}(s)\right| \leq C A e^{-\gamma \frac{s}{2}}+\frac{C A^{5}}{s^{3 / 2}}, \\
& \left|\hat{q}_{2}(s)\right| \leq C A e^{-\gamma \frac{s}{2}}+\frac{C}{s}, \\
& \left|\tilde{q}_{j}(s)\right| \leq C A e^{-\gamma \frac{s}{2}}+\frac{C A^{j-1}}{s^{\frac{j+1}{4}}} \text { for all } 3 \leq j \leq M, \\
& \left|\hat{q}_{j}(s)\right| \leq C A e^{-\gamma \frac{s}{2}}+\frac{C A^{j-1}}{s^{\frac{j+1}{4}}} \text { for all } 3 \leq j \leq M, \\
& \left\|\frac{q_{-}(s)}{1+|y|^{M+1}}\right\|_{L^{\infty}} \leq C \frac{A}{\left(\frac{s}{2}\right)^{\frac{M}{4}+2}}+C \frac{A^{M}}{s^{\frac{M+2}{4}}}, \\
& \left\|q_{e}(s)\right\|_{L^{\infty}} \leq \frac{C A^{M+1}}{\left(\frac{s}{2}\right)^{\frac{1}{4}}}(1+\log A) .
\end{aligned}
$$

Thus, if $A \geq A_{6}$ and $s_{0} \geq s_{6}(A)$ (that is $T \leq e^{-s_{6}(A)}$ ) for some positive $A_{6}$ and $s_{6}(A)$, we see that (67) holds.

Case 2: $s>s_{0}+\sigma$.

Let $\tau=s-\sigma>s_{0}$. Applying (iv) of Proposition 4.10 and using the fact that $q(\tau) \in \mathcal{V}_{A}(\tau)$, we write (we use (70) to bound any function of $\tau$ by a function of $s$ )

$$
\begin{aligned}
& \left|\hat{q}_{1}(s)\right| \leq e^{-\frac{\sigma}{2}} \frac{A^{6}}{\left(\frac{s}{2}\right)^{3 / 2}}+\frac{C A^{5}}{s^{3 / 2}}, \\
& \left|\hat{q}_{2}(s)\right| \leq e^{-\sigma} \frac{A^{2}}{\left(\frac{s}{2}\right)}+\frac{C}{s}, \\
& \left|\tilde{q}_{j}(s)\right| \leq e^{-\frac{(j-2) \sigma}{2}} \frac{A^{j}}{\left(\frac{s}{2}\right)^{\frac{j+1}{4}}}+\frac{C A^{j-1}}{s^{\frac{j+1}{4}}} \text { for all } 3 \leq j \leq M, \\
& \left|\hat{q}_{j}(s)\right| \leq e^{-\frac{j \sigma}{2}} \frac{A^{j}}{\left(\frac{s}{2}\right)^{\frac{j+1}{4}}}+\frac{C A^{j-1}}{s^{\frac{j+1}{4}}} \text { for all } 3 \leq j \leq M, \\
& \left\|\frac{q_{-}(s)}{1+|y|^{M+1}}\right\|_{L^{\infty}} \leq e^{-\frac{M+1}{4} \sigma} \frac{A^{M+1}}{\left(\frac{s}{2}\right)^{\frac{M+2}{4}}}+C \frac{A^{M}}{s^{\frac{M+2}{4}}}, \\
& \left\|q_{e}(s)\right\|_{L^{\infty}} \leq e^{-\frac{\sigma}{2(p-1)}} \frac{A^{M+2}}{\left(\frac{s}{2}\right)^{\frac{1}{4}}}+\frac{C A^{M+1}}{\left(\frac{s}{2}\right)^{\frac{1}{4}}}(1+\sigma) .
\end{aligned}
$$

For all the coordinates, it is clear that if $A \geq A_{7}$ and $s_{0} \geq s_{7}(A)$ for some positive $A_{7}$ and $s_{7}(A)$, then (66) and (67) is satisfied (remember that $\sigma=\log A$ ).

Conclusion of (ii): If $A \geq \max \left(A_{6}, A_{7}, A_{8}\right)$ and $s_{0} \geq \max \left(s_{6}(A), s_{7}(A), s_{8}(A)\right)$, then (67) is satisfied. Since we know that $q\left(s_{1}\right) \in \partial V_{A}\left(s_{1}\right)$, we see from the definition of $V_{A}(s)$ that $\left(\tilde{q}_{0}\left(s_{1}\right), \tilde{q}_{1}\left(s_{1}\right)\right) \in \partial\left[-\frac{A}{s_{1}^{3 / 2}}, \frac{A}{s_{1}^{3 / 2}}\right]^{2}$. This concludes the proof of (ii) of Proposition 4.7.

(iii) From (ii), there is $m=0,1$ and $\omega= \pm 1$ such that $\tilde{q}_{m}\left(s_{1}\right)=\omega \frac{A}{s_{1}^{3 / 2}}$.

Using (ii) of Proposition 4.7, we see that for $m=0$ or 1

$$
\omega \tilde{q}_{m}^{\prime}\left(s_{1}\right) \geq\left(1-\frac{m}{2}\right) \omega \tilde{q}_{m}\left(s_{1}\right)-\frac{C}{s_{1}^{3 / 2}} .
$$

Taking $A$ large enough gives $\omega \tilde{q}_{m}^{\prime}\left(s_{1}\right)>0$, for $m=0,1$ and concludes the proof of Proposition 4.7. 


\subsection{Proof of Proposition 4.10}

In this section, we prove Proposition 4.10. We just have to project equations (38) and (42) to get equations satisfied by the different coordinates of the decomposition (53). We proceed as Section 5 in [MZ08], taking into account the new scaling law $\frac{y}{s^{1 / 4}}$. We note that the projections of $V_{1} q+V_{2} \bar{q}, B$ and $R^{*}$ in (43), will need much more effort and this is due to the fact that we are dealing with the critical case.

More precisely, the proof will be carried out in 3 subsections

- In the first subsection, we deal with equation (42) to write equations satisfied by $\tilde{q}_{j}$ and $\hat{q}_{j}$. Then, we prove (i), (ii), (iii) and (iv) (expect the two last identities) of Proposition 4.10 .

- In the second subsection, we first derive from equation (42) an equation satisfied by $q_{-}$and prove the last but one identity in (iv) of Proposition 4.10 .

- In the third subsection, we project equation (38) (which is simpler than (42)) to write an equation satisfied by $q_{e}$ and prove the last identity in (iv) of Proposition 4.10 .

\subsubsection{The finite dimensional part $q_{+}$}

We proceed in 2 parts:

- In Part 1, we project equation (42) to get equations satisfied by $\tilde{q}_{j}$ and $\hat{q}_{j}$.

- In Part 2, we prove (i), (ii) and (iii) of Proposition 4.10, together with the estimates concerning $\tilde{q}_{j}$ and $\hat{q}_{j}$ in (iv).

Part 1: The projection of equation (42) on the eigenfunctions of the operator $\tilde{\mathcal{L}}$ In the following, we will find the main contribution in the projections $\tilde{P}_{n, M}$ and $\hat{P}_{n, M}$

of the six terms appearing in equation (42): $\partial_{s} q, \tilde{\mathcal{L}} q,-i\left(\frac{\mu}{s}+\theta^{\prime}(s)\right) q, V_{1} q+V_{2} \bar{q}, B(q, y, s)$ and $R^{*}\left(\theta^{\prime}, y, s\right)$. Most of the time, we give two estimates of error terms, depending on whether we use or not the fact that $q(s) \in \mathcal{V}_{A}(s)$.

First term: $\frac{\partial q}{\partial_{s}}$.

From (52), its projection on $\tilde{h}_{n}$ and $\hat{h}_{n}$ is $\tilde{q}_{n}^{\prime}$ and $\hat{q}_{n}^{\prime}$ respectively:

$$
\tilde{P}_{n}\left(\frac{\partial q}{\partial s}\right)=\tilde{q}_{n}^{\prime} \text { and } \hat{P}_{n}\left(\frac{\partial q}{\partial s}\right)=\hat{q}_{n}^{\prime}
$$

Second term: $\tilde{\mathcal{L}} q$.

We can easily see that

$$
\begin{aligned}
& \tilde{P}_{n}(\tilde{\mathcal{L}} q)=\left(1-\frac{n}{2}\right) \tilde{q}_{n}, \\
& \hat{P}_{n}(\tilde{\mathcal{L}} q)=-\frac{n}{2} \hat{q}_{n} .
\end{aligned}
$$

Third term: $-i\left(\frac{\mu}{s}+\theta^{\prime}\right) q$. It is enough to project $i q$, from (152), we have

$$
\begin{aligned}
& \tilde{P}_{n}\left(-i\left(\frac{\mu}{s}+\theta^{\prime}\right) q\right)=-\left(\frac{\mu}{s}+\theta^{\prime}(s)\right)\left(-\hat{q}_{n}-\delta \tilde{q}_{n}\right), \\
& \hat{P}_{n}\left(-i\left(\frac{\mu}{s}+\theta^{\prime}\right) q\right)=-\left(\frac{\mu}{s}+\theta^{\prime}(s)\right)\left(\delta \hat{q}_{n}+\left(1+\delta^{2}\right) \tilde{q}_{n}\right) .
\end{aligned}
$$


If in addition $q(s) \in \mathcal{V}_{A}(s)$, then the error estimates can be bounded from Definition 4.3 as follows:

Corollary 4.1 For all $A \geq 1$, there exists $s_{10}(A) \geq 1$ such that for all $s \geq s_{10}(A)$, if $q \in \mathcal{V}_{A}(s)$ and $\left|\theta^{\prime}(s)\right| \leq \frac{C A^{5}}{s^{3 / 2}}$, then:

a) for all $1 \leq n \leq M$, we have

$$
\left|\hat{P}_{n}\left(-i\left(\frac{\mu}{s}+\theta^{\prime}\right) q\right)\right| \leq C \frac{A^{n}}{s^{\frac{n+5}{4}}} .
$$

b) for $1 \leq n \leq M$, we have

$$
\left|\tilde{P}_{n}\left(-i\left(\frac{\mu}{s}+\theta^{\prime}\right) q\right)\right| \leq C \frac{A^{n}}{s^{\frac{n+5}{4}}}
$$

c) for $n=0,\left|\tilde{P}_{0}\left(-i\left(\frac{\mu}{s}+\theta^{\prime}\right) q\right)\right|+\left|\hat{P}_{0}\left(-i\left(\frac{\mu}{s}+\theta^{\prime}\right) q\right)\right| \leq \frac{C}{s^{3 / 2}}$

Fourth term: $V_{1} q+V_{2} \bar{q}$.

We claim the following

Lemma 4.12 (Projection of $V_{1} q$ and $V_{2} \bar{q}$ ) (i) It holds that

$$
\left|V_{i}(y, s)\right| \leq C \frac{\left(1+|y|^{2}\right)}{s^{1 / 2}}, \text { for all } y \in \mathbb{R} \text { and } s \geq 1,
$$

and for all $k \in \mathbb{N}^{*}$

$$
V_{i}(y, s)=\sum_{j=1}^{k} \frac{1}{s^{j / 2}} W_{i, j}(y)+\tilde{W}_{i, k}(y, s),
$$

where $W_{i, j}$ is an even polynomial of degree $2 j$ and $\tilde{W}_{i, k}(y, s)$ satifies

$$
\text { for all } s \geq 1 \text { and }|y| \leq s^{1 / 4},\left|\tilde{W}_{i, k}(y, s)\right| \leq C \frac{\left(1+|y|^{2 k+2}\right)}{s^{\frac{k+1}{2}}} \text {. }
$$

(ii) The projection of $V_{1} q$ and $V_{2} \bar{q}$ on $(1+i \delta) h_{n}$ and $i h_{n}$, and we have

$$
\begin{aligned}
& \left|\tilde{P}_{n}\left(V_{1} q\right)\right|+\left|\hat{P}_{n}\left(V_{1} q\right)\right| \\
& \leq \frac{C}{s^{1 / 2}} \sum_{j=n-2}^{M}\left(\left|\tilde{q}_{j}\right|+\left|\hat{q}_{j}\right|\right)+\sum_{j=0}^{n-3} \frac{C}{s^{\frac{n-j}{4}}}\left(\left|\tilde{q}_{j}\right|+\left|\hat{q}_{j}\right|\right)+\frac{C}{s^{1 / 2}}\left\|\frac{q-}{1+|y|^{M+1}}\right\|_{L^{\infty}},
\end{aligned}
$$

and the same holds for $V_{2} \bar{q}$

Remark 4.13 If $n \leq 2$, the first sum in (79) runs for $j=0$ to $M$ and the second sum doesn't exist.

If in addition $q(s) \in \mathcal{V}_{A}(s)$, then the error estimates can be bounded from Definition 4.3 as follows: 
Corollary 4.2 For all $A \geq 1$, there exists $s_{11}(A) \geq 1$ such that for all $s \geq s_{11}(A)$, if $q \in \mathcal{V}_{A}(s)$, then:

a) for $3 \leq n \leq M$, we have

$$
\left|\tilde{P}_{n}\left(V_{1} q\right)\right|+\left|\hat{P}_{n}\left(V_{1} q\right)\right| \leq C \frac{A^{n-2}}{s^{\frac{n+1}{4}}}
$$

b) for $n=0,1$ or 2 , we have

$$
\left|\tilde{P}_{n}\left(V_{1} q\right)\right|+\left|\hat{P}_{n}\left(V_{1} q\right)\right| \leq \frac{C A^{5}}{s^{\frac{3}{2}}}
$$

Proof of Lemma 4.12:

(i) The estimates of $V_{1} q$ and $V_{2} \bar{q}$ are the same, so we only deal with $V_{1} q$. Let $F(u)=$ $\frac{(p+1)}{2}(1+i \delta)\left[|u|^{p-1}-\frac{1}{p-1}\right]$, where $u \in \mathbb{C}$ and consider $z=\frac{y}{s^{1 / 4}}$. Note that from (42) and (37), we have

$$
V_{1}(y, s)=F(\varphi(y, s)), \text { where } \varphi(y, s)=\varphi_{0}\left(\frac{y}{s^{1 / 4}}\right)+\frac{a}{s^{1 / 2}}(1+i \delta) .
$$

Note that there exist positive constant $c_{0}$ and $s_{0}$ such that $\varphi_{0}(z) \mid$ and $|\varphi(y, s)|=\mid \varphi_{0}\left(\frac{y}{s^{1 / 4}}\right)+$ $\frac{a}{s^{1 / 2}}(1+i \delta) \mid$ are both larger than $\frac{1}{c_{0}}$ and smaller than $c_{0}$, uniformly in $|z|<1$ and for $s \geq s_{0}$. Since $F(u)$ is $C^{\infty}$ for $\frac{1}{c_{0}} \leq|u| \leq c_{0}$, we expand it around $u=\varphi_{0}(z)$ as follows: for all $s \geq s_{0}$ and $|z|<1$,

$$
\left|\begin{array}{l}
F\left(\varphi_{0}(z)+\frac{a}{s^{1 / 2}}(1+i \delta)\right)-F\left(\varphi_{0}(z)\right) \mid \leq \frac{C}{s^{1 / 2}}, \\
F\left(\varphi_{0}(z)+\frac{a}{s^{1 / 2}}(1+i \delta)\right)-F\left(\varphi_{0}(z)\right)-\sum_{j=1}^{n} \frac{1}{s^{j / 2}} F_{j}\left(\varphi_{0}(z)\right)
\end{array}\right| \leq \frac{C}{s^{\frac{n+1}{2}}},
$$

where $F_{j}(u)$ are $C^{\infty}$. Hence, we can expand $F(u)$ and $F_{j}(u)$ around $u=\varphi_{0}(0)$ and write for all $s \geq s_{0}$ and $|z|<1$,

$$
\begin{aligned}
& \left|F\left(\varphi_{0}(z)+\frac{a}{s^{1 / 2}}(1+i \delta)\right)-F\left(\varphi_{0}(0)\right)\right| \leq C z^{2}+\frac{C}{s^{1 / 2}}, \\
& F\left(\varphi_{0}(z)+\frac{a}{s^{1 / 2}}(1+i \delta)\right)-F\left(\varphi_{0}(0)\right)-\sum_{l=1}^{n} c_{0, l} z^{2 l}-\sum_{j=1}^{n} \sum_{l=0}^{n-j} \frac{c_{j, l}}{s^{j / 2}} z^{2 l} \mid \\
& \leq C|z|^{2 n+2}+\sum_{j=1}^{n} \frac{C}{j^{1 / 2}}|z|^{2(n-j)+2} \frac{C}{s^{\frac{n+1}{2}}} .
\end{aligned}
$$

Since $F\left(\varphi_{0}(0)\right)=F(\kappa)=0$ and $z=\frac{y}{s^{1 / 4}}$, this gives us estimates in (i), when $s \geq s_{0}$ and $|y|<s^{1 / 4}$. Since $V_{1}$ is bounded, the inequalities still valid when $|y| \geq s^{1 / 4}$ and then when $s \geq 1$.

(ii) Note first that it is enough to prove the bound (51) for the projection of $V_{i} q$ onto $h_{n}$ to get the same bound for $\tilde{P}_{n}\left(V_{i} q\right)$ and $\hat{P}_{n}\left(V_{i} q\right)$. Since in addition, the proof for $V_{2} \bar{q}$ is 
the same as for $V_{1} q$, we only prove (179) for the projection of $V_{1} q$ onto $h_{n}$. Using (53) and the fact that $\tilde{h}_{n}=(1+i \delta) h_{n}$ and $\hat{h}_{n}=i h_{n}$, we see that the projection is given by

$$
\begin{aligned}
\int h_{n} V_{1} q \rho & =\int h_{n} V_{1} q_{-} \rho+\sum_{j=0}^{M} \tilde{q}_{j} \int h_{n} \tilde{h}_{j} V_{1} \rho+\sum_{j=0}^{M} \hat{q}_{j} \int h_{n} \hat{h}_{j} V_{1} \rho . \\
& =\int h_{n} V_{1} q_{-} \rho+(1+i \delta) \sum_{j=0}^{M} \tilde{q}_{j} \int h_{n} h_{j} V_{1} \rho+i \sum_{j=0}^{M} \hat{q}_{j} \int h_{n} h_{j} V_{1} \rho
\end{aligned}
$$

The first term can be bounded by

$$
\int h_{n} V_{1}\left(\frac{1+|y|^{2}}{s^{1 / 2}}\right)\left|q_{-}\right| \rho \leq \frac{C}{s^{1 / 2}}\left\|\frac{q_{-}}{1+|y|^{M+1}}\right\|_{L^{\infty}} .
$$

Now we deal with the second term. We only focus on the terms involving $h_{j}$.

If $j \geq n-2$, we use (76) to write $\left|\int h_{n} h_{j} V_{1} \rho\right| \leq \frac{C}{s^{1 / 2}}$.

If $j \leq n-3$, then we claim that

$$
\left|\int h_{n} h_{j} V_{1} \rho\right| \leq \frac{C}{s^{\frac{n-j}{4}}}
$$

(this actually vanishes if $j$ and $n$ have different parities). It is clear that (79) follows from (80), eqrefbd2 and (82).

Let us prove (82). Note that $k \equiv\left[\frac{n-j-1}{2}\right]$ (which is in $\mathbb{N}^{*}$ since $j \leq n-3$ ) is the largest integer such that $j+2 k<n$. We use (77) to write

$$
\begin{aligned}
& \int h_{n} h_{j} V_{1} \rho=\int_{|y|<s^{1 / 4}} h_{n} h_{j} V_{1} \rho+\int_{|y|>s^{1 / 4}} h_{n} h_{j} V_{1} \rho, \\
& =\sum_{l=1}^{k} \frac{1}{s^{l / 2}} \int_{|y|<s^{1 / 4}} h_{n} h_{j} W_{1, l} \rho+O\left(\frac{1}{s^{\left.\frac{\left[-n^{-1}\right.}{2}\right]+1}} \int\left(1+|y|^{n-j+1}\right)\left|h_{n}\right|\left|h_{j}\right| \rho d y\right)+\int_{|y|>s^{1 / 4}} h_{n} h_{j} V_{1} \rho, \\
& =\sum_{l=1}^{k} \frac{1}{s^{l / 2}} \int_{\mathbb{R}^{N}} h_{n} h_{j} W_{1, l} \rho+O\left(\frac{1}{\frac{\left[\frac{n-j-1}{2}\right]+1}{2}}\right)-\sum_{l=1}^{k} \frac{1}{s^{l}} \int_{|y|>s^{1 / 4}} h_{n} h_{j} W_{1, l} \rho+\int_{|y|>s^{1 / 4}} h_{n} h_{j} V_{1} \rho,
\end{aligned}
$$

since $\operatorname{deg}\left(h_{j} W_{1, l}\right)=j+2 l \leq j+2 k<n=\operatorname{deg}\left(h_{n}\right), h_{n}$ is orthogonal to $h_{j} W_{1, l}$ and

$$
\int_{\mathbb{R}^{N}} h_{n} h_{j} W_{1, l} \rho=0 .
$$

Since $|\rho(y)| \leq C e^{-c s^{1 / 2}}$ when $|y|>s^{1 / 4}$, the integrals over the domain $|y|>s^{1 / 4}$ can be bounded by

$$
C e^{-c s^{1 / 2}} \int_{|y|>s^{1 / 4}}\left|h_{n}\right|\left|h_{j}\right|\left(1+|y|^{2 k}\right) \sqrt{\rho} \leq C e^{-c s^{1 / 2}} .
$$

Using that $\left[\frac{n-j-1}{2}\right]+1 \geq \frac{n-j}{2}$, we deduce that (82) holds. Hence, we have proved (79) and this concludes the proof of Lemma 4.12.

We need further refinements when $n=0,2$ for the terms $\tilde{P}_{2, M}\left(V_{1} q\right), \tilde{P}_{2, M}\left(V_{2} \bar{q}\right)$, $\hat{P}_{0, M}\left(V_{1} q\right)$ and $\hat{P}_{0, M}\left(V_{2} \bar{q}\right)$. More precisely 
Lemma 4.14 Projection of $V_{1} q$ and $V_{2} \bar{q}$ on $\hat{h}_{0}$ and $\tilde{h}_{2}$

(i) It holds that for $i=1,2$

$$
\forall s \geq 1 \text { and }|y|<s^{1 / 4},\left|V_{i}(y, s)-\frac{1}{s^{1 / 2}} W_{i, 1}(y)-\frac{1}{s} W_{i, 2}(y)\right| \leq \frac{C}{s^{3 / 2}}\left(1+|y|^{6}\right),
$$

where

$$
\begin{aligned}
& W_{1,1}=-(1+i \delta) \frac{b(p+1)}{2(p-1)^{2}} h_{2}(y) \\
& W_{1,2}=(1+i \delta) \frac{b^{2}(p+1)}{2(p-1)^{3}} h_{2}^{2}(y) \\
& W_{2,1}=-(1+i \delta) \frac{p-1}{2} \frac{b}{(p-1)^{3}}(p-1+2 i \delta) h_{2}(y) \\
& W_{2,2}=(1+i \delta) \frac{b^{2}}{2(p-1)^{3}}\left[\left(p^{2}-4 p+1\right) h_{2}^{2}(y)+i \delta\left(8(p-2)\left(1-y^{2}\right)+y^{4} 3(p-1)\right)\right] .
\end{aligned}
$$

(ii) The projection of $V_{1} q$ and $V_{2} \bar{q}$ on $\tilde{h}_{2}$ satisfy

$$
\begin{aligned}
& \mid \tilde{P}_{2}\left(V_{1} q\right)+\frac{1}{s^{1 / 2}}\left[\sum_{j \geq 0} \tilde{A}_{j}^{1} \tilde{q}_{j}+\sum_{j \geq 0} \hat{B}_{j}^{1} \hat{q}_{j}\right]+\frac{\tilde{q}_{2}}{s} 60 b^{2}(p+1) \frac{1}{(p-1)^{2}} \\
& +\tilde{P}_{2}\left(V_{2} \bar{q}\right)+\frac{1}{s^{1 / 2}}\left[\sum_{j \geq 0} \tilde{A}_{j}^{2} \tilde{q}_{j}+\sum_{j \geq 0} \hat{B}_{j}^{2} \hat{q}_{j}\right]-\frac{\tilde{q}_{2}}{s} 60 b^{2}(p+1) \frac{p^{2}-4 p+1}{2(p-1)^{3}} \mid \\
& \leq \frac{C}{s} \sum_{j=0, j \neq 2}^{M}\left|\tilde{q}_{j}\right|+\frac{C}{s} \sum_{j=0}^{M}\left|\hat{q}_{j}\right|+\frac{C}{s}|| \frac{q_{-}}{1+|y|^{M}} \|_{L^{\infty}}+\frac{C}{s^{3 / 2}}\left|\tilde{q}_{2}(s)\right| .
\end{aligned}
$$

where, for all $j \geq 0$

$$
\begin{array}{ll}
\tilde{A}_{j}^{1}=\tilde{P}_{2}\left(W_{1,1} \tilde{h}_{j}\right) & \hat{B}_{j}^{1}=\tilde{P}_{2}\left(W_{1,1} \hat{h}_{j}\right) \\
\tilde{A}_{j}^{2}=\tilde{P}_{2}\left(W_{2,1} \tilde{\tilde{h}}_{j}\right) & \hat{B}_{j}^{2}=\tilde{P}_{2}\left(W_{2,1} \overline{\hat{h}}_{j}\right),
\end{array}
$$

and

$$
\tilde{A}_{j}^{1}=-\tilde{A}_{j}^{2}, \quad \hat{B}_{j}^{1}=-\hat{B}_{j}^{2}
$$

ii) The projection of $V_{1} q$ and $V_{2} \bar{q}$ on $\hat{h}_{0}$ satisfy

$$
\begin{aligned}
& \left|\hat{P}_{0}\left(V_{1} q\right)+\frac{\tilde{q}_{2}}{s^{1 / 2}} 4 \delta b(p+1) \frac{(p+1)}{(p-1)^{2}}\right| \\
& \left.+\mid \hat{P}_{0}\left(V_{2} \bar{q}\right)\right)-\frac{\tilde{q}_{2}}{s^{1 / 2}} 4 \delta b(p+1) \frac{(p-3)}{(p-1)^{2}} \mid \\
& \leq \frac{C}{s^{1 / 2}} \sum_{j=0, j \neq 2}^{M}\left|\tilde{q}_{j}\right|+\frac{C}{s^{1 / 2}} \sum_{j=0}^{M}\left|\hat{q}_{j}\right|+\frac{C}{s^{1 / 2}} \|\left.\frac{q_{-}}{1+|y|^{M}}\right|_{L^{\infty}}+\frac{C}{s^{3 / 2}}\left|\tilde{q}_{2}(s)\right| .
\end{aligned}
$$

In addition, if $q(s) \in \mathcal{V}_{A}(s)$, then the error estimates can be bounded from (56) as follows;

Corollary 4.3 For all $A \geq 1$, there exists $s_{12}(A) \geq 1$ such that for all $s \geq s_{12}(A)$, if $q(s) \in \mathcal{V}_{A}(s)$, then

$$
\left|\tilde{P}_{2}\left(V_{1} q\right)+\frac{\tilde{q}_{2}}{s} 60 b^{2}(p+1) \frac{1}{(p-1)^{2}}+\tilde{P}_{2}\left(V_{2} \bar{q}\right)-\frac{\tilde{q}_{2}}{s} 60 b^{2}(p+1) \frac{p^{2}-4 p+1}{2(p-1)^{3}}\right| \leq C \frac{A^{3}}{s^{2}}
$$




$$
\begin{aligned}
& \left|\hat{P}_{0}\left(V_{1} q\right)+\frac{\tilde{q}_{2}}{s^{1 / 2}} 4 \delta b(p+1) \frac{(p+1)}{(p-1)^{2}}\right| \leq C \frac{A^{3}}{s^{3 / 2}} \\
& \left.\mid \hat{P}_{0}\left(V_{2} \bar{q}\right)\right)-\frac{\tilde{q}_{2}}{s^{1 / 2}} 4 \delta b(p+1) \frac{(p-3)}{(p-1)^{2}} \mid \leq C \frac{A^{3}}{s^{3 / 2}}
\end{aligned}
$$

Proof of Lemma 4.14: (i) This is a simple, but lengthy computation that we omit. For more details see Appendix B.

(ii) Using (84) and (53), we see that

$$
\begin{aligned}
V_{1} q & =\frac{1}{s^{1 / 2}} W_{1,1} q+\frac{1}{s} W_{1,2} q+O\left(\frac{q\left(1+|y|^{6}\right)}{s^{3 / 2}}\right) \\
& =\frac{1}{s^{1 / 2}} W_{1,1}\left(\sum_{j=0}^{M} \tilde{q}_{j} \tilde{h}_{j}+\sum_{j=0}^{M} \hat{q}_{j} \hat{h}_{j}\right) \\
& +\frac{1}{s} W_{1,2}\left(\sum_{j=0}^{M} \tilde{q}_{j} \tilde{h}_{j}+\sum_{j=0}^{M} \hat{q}_{j} \hat{h}_{j}\right)+O\left(\frac{q\left(1+|y|^{6}\right)}{s^{3 / 2}}\right),
\end{aligned}
$$

where $O$ is uniform with respect to $|y|<s^{1 / 4}$. By the definition of the components of $q$ :

$$
|q(y, s)| \leq\left[\sum_{i=1}^{M}\left(\left|\tilde{q}_{i}\right|+\left|\hat{q}_{i}\right|\right)+\frac{\|q(s)\|_{L^{\infty}}}{1+|y|^{M}}\right]\left(1+|y|^{M}\right),
$$

and we may replace the occurrence of $q$ by its components.

When projecting (88) on $\tilde{h}_{2}$ (use (52) for the definition of that projection), we write using the definition (85) of $W_{1,1}$ and $W_{1,2}$ and (89)

$$
\begin{aligned}
& \mid \tilde{P}_{2}\left(V_{1} q\right)-\frac{1}{s^{1 / 2}}\left[\sum_{j \geq 0} \tilde{P}_{2}\left(W_{1,1} \tilde{h}_{j}\right) \tilde{q}_{j}+\sum_{j \geq 0} \tilde{P}_{2}\left(W_{1,1} \hat{h}_{j}\right) \hat{q}_{j}\right]-\frac{\tilde{q}_{2}(s)}{s} \frac{b^{2}(p+1)}{2(p-1)^{3}} \tilde{P}_{2}\left(\left(\tilde{h}_{2}\right)^{2} h_{2}\right) \\
& +\tilde{P}_{2}\left(V_{2} \bar{q}\right)-\frac{1}{s^{1 / 2}}\left[\sum_{j \geq 0} \tilde{P}_{2}\left(W_{2,1} \tilde{h}_{j}\right) \tilde{q}_{j}+\sum_{j \geq 0} \tilde{P}_{2}\left(W_{2,1} \hat{h}_{j}\right) \hat{q}_{j}\right]-\frac{\tilde{q}_{2}(s)}{s} \frac{b^{2}(p+1)\left(p^{2}-4 p+1\right)}{2(p-1)^{3}} \tilde{P}_{2}\left(h_{2}^{3}\right) \mid \\
& \leq \frac{C}{s^{1 / 2}} \sum_{j=0, \neq 2}^{M}\left|\tilde{q}_{j}\right|+\frac{C}{s^{1 / 2}} \sum_{j=0}^{M}\left|\hat{q}_{j}\right|+\frac{C}{s^{1 / 2}}\left\|\frac{q_{-}}{1+|y|^{M}}\right\|_{L^{\infty}}+\frac{C}{s^{3 / 2}}\left|\tilde{q}_{2}(s)\right|
\end{aligned}
$$

We note by (85)

$$
\begin{aligned}
& W_{1,1} \tilde{h}_{j}=[(p-1)(1+i \delta)-i \delta(p+1)] \frac{b(p+1)}{2(p-1)^{2}} h_{j} h_{2} \\
& W_{2,1} \tilde{h}_{j}=[-(p-1)(1+i \delta)+i \delta(p-3)] \frac{b(p+1)}{2(p-1)^{2}} h_{j} h_{2} \\
& W_{1,1} \hat{h}_{j}=[\delta(1+i \delta)-i(p+1)] \frac{b(p+1)}{2(p-1)^{2}} h_{j} h_{2} \\
& W_{2,1} \hat{h}_{j}=[-\delta(1+i \delta)+i(p-1)] \frac{b(p+1)}{2(p-1)^{2}} h_{j} h_{2}
\end{aligned}
$$

and we deduce that for all $k \geq 0$

$$
\begin{aligned}
& \tilde{P}_{k}\left(W_{1,1} \tilde{h}_{j}\right)=-\tilde{P}_{k}\left(W_{2,1} \overline{\tilde{h}}_{j}\right) \\
& \tilde{P}_{k}\left(W_{1,1} \hat{h}_{j}\right)=-\tilde{P}_{k}\left(W_{2,1} \hat{\hat{h}}_{j}\right),
\end{aligned}
$$


In particular when $k=2$, terms involving $\frac{\tilde{q}_{j}}{s^{1 / 2}}$ and $\frac{\hat{q}_{j}}{s^{1 / 2}}$ disappear, it happens that its occurrence coming from $\tilde{P}_{2}\left(V_{1} q\right)$ and $\tilde{P}_{2}\left(V_{2} \bar{q}\right)$ do cancels.

Therefore, the problem is reduced to the projection of $\left(\tilde{h}_{2}\right)^{2} h_{2}$ and $h_{2}^{3}$ on $\tilde{h}_{2}$

$$
\tilde{P}_{2}\left(\left(\tilde{h}_{2}\right)^{2} h_{2}\right)=120(1-p), \quad \tilde{P}_{2}\left(\tilde{h}_{2}^{3}\right)=120 .
$$

The other bounds on(86) and (87) are similar, thus we skip it.

Remark 4.15 From equation (91), we can see that terms involving $\frac{\tilde{q}_{j}}{s^{1 / 2}}$ and $\frac{\hat{q}_{j}}{s^{1 / 2}}$ disappear on $\tilde{P}_{k}\left(V_{1} q\right)+\tilde{P}_{k}\left(V_{2} \bar{q}\right)$. Then, arguing as in Lemma 4.12 and Corollary 4.2 we can obtain a better estimate on $\left|\tilde{P}_{k}\left(V_{1} q\right)+\tilde{P}_{k}\left(V_{2} \bar{q}\right)\right|$, for all $k \geq 0$ :

$$
\left|\tilde{P}_{k}\left(V_{1} q\right)+\tilde{P}_{k}\left(V_{2} \bar{q}\right)\right| \leq \frac{C}{s^{3 / 2}},
$$

Fifth term: $B(q, y, s)$ Let us recall from (43) that:

$$
B(q, y, s)=(1+i \delta)\left(|\varphi+q|^{p-1}(\varphi+q)-|\varphi|^{p-1} \varphi-|\varphi|^{p-1} q-\frac{p-1}{2}|\varphi|^{p-3} \varphi(\varphi \bar{q}+\bar{\varphi} q)\right) .
$$

We have the following

Lemma 4.16 The function $B=B(q, y, s)$ can be decomposed for all $s \geq 1$ and $|q| \leq 1$ as

$$
\sup _{|y|<s^{1 / 4}}\left|B-\sum_{\substack{l=0 \\ 0 \leq j, k \leq M+1 \\ 2 \leq j+k \leq M+1}}^{M} \frac{1}{s^{l / 2}}\left[B_{j, k}^{l}\left(\frac{y}{s^{1 / 4}}\right) q^{j} \bar{q}^{k}+\tilde{B}_{j, k}^{l}(y, s) q^{j} \bar{q}^{k}\right]\right| \leq C|q|^{M+2}+\frac{C}{s^{\frac{M+1}{2}}}
$$

where $B_{j, k}^{l}\left(\frac{y}{s^{1 / 4}}\right)$ is an even polynomial of degree less or equal to $M$ and the rest $\tilde{B}_{j, k}^{l}(y, s)$ satisfies

$$
\forall s \geq 1 \text { and }|y|<s^{1 / 4},\left|\tilde{B}_{j, k}^{l}(y, s)\right| \leq C \frac{1+|y|^{M+1}}{s^{\frac{M+1}{2}}}
$$

Moreover,

$$
\forall s \geq 1 \text { and }|y|<s^{1 / 4},\left|B_{j, k}^{l}\left(\frac{y}{s^{1 / 4}}\right)+\tilde{B}_{j, k}^{l}(y, s)\right| \leq C .
$$

On the other hand, in the region $|y| \geq s^{1 / 4}$, we have

$$
|B(q, y, s)| \leq C|q|^{\bar{p}},
$$

for some constant $C$ where $\bar{p}=\min (p, 2)$.

Proof: See the proof of Lemma 5.9, page 1646 in [MZ08. 
Lemma 4.17 (The quadratic term $B(q, y, s)$ ) For all $A \geq 1$, there exists $s_{13}$ such that for all $s \geq s_{13}$, if $q(s) \in \mathcal{V}_{A}(s)$, then:

a) the projection of $B(q, y, s)$ on $(1+i \delta) h_{n}$ and on $i h_{n}$, for $n \geq 3$ satisfies

$$
\left|\tilde{P}_{n}(B(q, y, s))\right|+\left|\hat{P}_{n}(B(q, y, s))\right| \leq C \frac{A^{n}}{s^{\frac{n+2}{4}}} .
$$

b) For $n=0,1$, we have

$$
\left|\tilde{P}_{n}(B(q, y, s))\right|+\left|\hat{P}_{n}(B(q, y, s))\right| \leq \frac{C}{s^{\frac{5}{2}}}
$$

and

$$
\left|\tilde{P}_{2}(B(q, y, s))\right|+\left|\hat{P}_{2}(B(q, y, s))\right| \leq \frac{C}{s^{\frac{5}{2}}}
$$

Proof : We only prove estimate (95), since (96) can be proved in the same way. The estimation (97), is also proved in the same way and using the Taylor expansion of $B$, established in Appendix D.

It is enough to prove estimate (95) for the projection on $h_{n}$ since it implies the same estimate on $\tilde{P}_{n}$ and $\hat{P}_{n}$ through (52). We have

$$
\int h_{n} B(q, y, s) \rho d y=\int_{|y|<s^{1 / 4}} h_{n} B(q, y, s) \rho d y+\int_{|y|>s^{1 / 4}} h_{n} B(q, y, s) \rho d y .
$$

Using Lemma 4.16, we deduce that

$$
\begin{aligned}
& \left|\int_{|y|<s^{1 / 4}} h_{n} B(q, y, s) \rho d y-\int_{|y|<s^{1 / 4}} h_{n} \rho \sum_{l=0}^{M} \sum_{\substack{0 \leq j, k \leq M+1 \\
2 \leq j+k \leq M+1}} \frac{1}{s^{l / 2}}\left[B_{j, k}^{l}\left(\frac{y}{s^{1 / 4}}\right) q^{j} \bar{q}^{k}+\tilde{B}_{j, k}^{l}(y, s) q^{j} \bar{q}^{k}\right]\right| \\
& \leq C \int_{|y|<s^{1 / 4}}\left|h_{n}\right||\rho|\left(|q|^{M+2}+\frac{1}{s^{\frac{M+1}{2}}}\right) .
\end{aligned}
$$

Let us write

$$
\begin{gathered}
B_{j, k}^{l}\left(\frac{y}{s^{1 / 4}}\right)=\sum_{i=0}^{M / 2} b_{j, k}^{l, i}\left(\frac{y}{s^{1 / 4}}\right)\left(\frac{y}{s^{1 / 4}}\right)^{2 i}, \\
q^{j}=\left(\sum_{m=0}^{M} \tilde{q}_{m} \tilde{h}_{m}+\hat{q}_{m} \hat{h}_{m}+q_{-}\right)^{j}, q^{k}=\left(\sum_{m=0}^{M} \tilde{q}_{m} \tilde{h}_{m}+\hat{q}_{m} \hat{h}_{m}+q_{-}\right)^{k},
\end{gathered}
$$

where $b_{j, k}^{l, i}$ are the coefficients of the polynomials $B_{j, k}^{l}$. Using the fact that $\|q(s)\|_{L^{\infty}} \leq 1$ (which holds for $s$ large enough, from the fact that $q(s) \in \mathcal{V}_{A}(s)$ and (i) of Proposition 56). We deduce that

$$
\left|q^{j}-q_{+}^{j}\right| \leq C\left(\left|q_{-}\right|^{j}+\left|q_{-}\right|\right)
$$


Using that $q(s) \in \mathcal{V}_{A}(s)$ and the fact that $\sqrt{s} \geq 2 A^{2}$, we deduce that in the region $|y| \leq s^{1 / 4}$, we have $\left|q_{-}\right| \leq \frac{1}{s^{1 / 4}}\left(\frac{A}{s^{1 / 4}}\right)^{M+1}(1+|y|)^{M+1}$ and that

$$
\left|q^{j}-\left(\sum_{m=0}^{M} \tilde{q}_{m} \tilde{h}_{m}+\hat{q}_{m} \hat{h}_{m}\right)^{j}\right| \leq C\left(\frac{A}{s^{1 / 4}}\right)^{M+1} \frac{1}{s^{1 / 4}}(1+|y|)^{j M+j} .
$$

In the same way, we have

$$
\left|q^{k}-\left(\sum_{m=0}^{M} \tilde{q}_{m} \tilde{h}_{m}+\hat{q}_{m} \hat{h}_{m}\right)^{k}\right| \leq C\left(\frac{A}{s^{1 / 4}}\right)^{M+1} \frac{1}{s^{1 / 4}}(1+|y|)^{k M+k},
$$

hence, the contribution coming from $q_{-}$is controlled by the right-hand side of (95). Moreover for all $j, k$ and $l$, we have

$$
\left|\int_{|y|<s^{1 / 4}} h_{n} \rho B_{j, k}^{l}\left(\frac{y}{s^{1 / 4}}\right) q_{+}^{j} \bar{q}_{+}^{k}-\int h_{n} \rho B_{j, k}^{l}\left(\frac{y}{s^{1 / 4}}\right) q_{+}^{j} \bar{q}_{+}^{k}\right| \leq C e^{-C \sqrt{s}} .
$$

To compute the second term on the left had side of (98), we notice that $B_{j, k}^{l}\left(\frac{y}{s^{1 / 4}}\right) q_{+}^{j} \bar{q}_{+}^{k}$ is a polynomial in $y$ and that the coefficient of the term of degree $n$ is controlled by the right had side of (95) since $q \in \mathcal{V}_{A}$.

Moreover, using that $\sqrt{s} \geq 2 A^{2}$, we infer that $|q| \leq \frac{1}{s^{1 / 4}}(1+|y|)^{M+1}$ in the region $|y| \leq s^{1 / 4}$ and hence for all $j, k$ and $l$, we have

$$
\left|\int_{|y|<s^{1 / 4}} h_{n} \rho \frac{1}{s^{l / 2}} \tilde{B}_{j, k}^{l}(y, s) q^{j} \bar{q}^{k}\right| \leq C \frac{1}{s^{\frac{l}{2}+\frac{M+1+j+k}{4}}}
$$

and

$$
\left|\int_{|y|<s^{1 / 4}} h_{n} \rho\left(|q|^{M+2}+\frac{1}{s^{\frac{M+1}{2}}}\right)\right| \leq C \frac{1}{s^{\frac{M+2}{4}}} .
$$

The terms appearing in these two inequalities are controlled by the right hand side of (95).

Using the fact that $\|q(s)\|_{L^{\infty}} \leq 1$ and (43), we remark that $|B(q, y, s)| \leq C$. Since $|\rho(y)| \leq C e^{-s \sqrt{s}}$ for all $|y|>s^{1 / 4}$, it holds that

$$
\left|\int_{|y|>s^{1 / 4}} h_{n} B(q, y, s) \rho d y\right| \leq C e^{-C \sqrt{s}}
$$

This concludes the proof of Lemma 4.17.

Sixth term: $R^{*}\left(\theta^{\prime}, y, s\right)$

In the following, we expand $R^{*}$ as a power series of $\frac{1}{s}$ as $s \rightarrow \infty$, uniformly for $|y| \leq s^{1 / 4}$.

Lemma 4.18 (Power series of $R^{*}$ as $s \rightarrow \infty$ ) For all $n \in \mathbb{N}$,

$$
R^{*}\left(\theta^{\prime}, y, s\right)=\Pi_{n}\left(\theta^{\prime}, y, s\right)+\tilde{\Pi}_{n}\left(\theta^{\prime}, y, s\right)
$$


where,

$$
\Pi_{n}\left(\theta^{\prime}, y, s\right)=\sum_{k=0}^{n-1} \frac{1}{s^{\frac{k+1}{2}}} P_{k}(y)-i \theta^{\prime}(s)\left(\frac{a}{s^{1 / 2}}(1+i \delta)+\sum_{k=0}^{n-1} e_{k} \frac{y^{2 k}}{s^{k / 2}}\right)
$$

and

$$
\forall|y|<s^{1 / 4}, \quad\left|\tilde{\Pi}_{n}\left(\theta^{\prime}, y, s\right)\right| \leq C\left(1+s\left|\theta^{\prime}(s)\right|\right) \frac{\left(1+|y|^{2 n}\right)}{s^{\frac{n+1}{2}}},
$$

where $P_{k}$ is a polynomial of order $2 k$ for all $k \geq 1$ and $e_{k} \in \mathbb{R}$.

In particular,

$$
\begin{aligned}
& \sup _{|y| \leq s^{1 / 4}}\left|R^{*}\left(\theta^{\prime}, y, s\right)-\sum_{k=0}^{1} \frac{1}{s^{\frac{k+1}{2}}} P_{k}(y)+i \theta^{\prime}\left[\kappa+\frac{(1+i \delta)}{s^{1 / 2}}\left(a-\frac{b \kappa y^{2}}{(p-1)^{2}}\right)\right]\right| \\
& \leq C\left(\frac{1+|y|^{4}}{s^{3 / 2}}+C\left|\theta^{\prime}\right| \frac{y^{4}}{s^{2}}\right) .
\end{aligned}
$$

Proof: Using the definition of $\varphi$ (34), the fact that $\varphi_{0}$ satisfies (37) and (42), we see that $R^{*}$ is in fact a function of $\theta^{\prime}, z=\frac{y}{s^{1 / 4}}$ and $s$ that can be written as

$$
\begin{aligned}
& R^{*}\left(\theta^{\prime}, y, s\right)=\frac{1}{4} \frac{z}{s} \nabla_{z} \varphi_{0}(z)+\frac{1}{2} \frac{a}{s^{3 / 2}}(1+i \delta)+\frac{1}{s^{1 / 2}} \Delta_{z} \varphi_{0}(z) \\
& -\frac{a(1+i \delta)^{2}}{(p-1) s^{1 / 2}}+\left(F\left(\varphi_{0}(z)+\frac{a}{s^{1 / 2}}(1+i \delta)\right)-F\left(\varphi_{0}\right)\right) \\
& -i \frac{\mu}{s}\left(\varphi_{0}(z)+\frac{a}{s^{1 / 2}}(1+i \delta)\right)-i \theta^{\prime}(s)\left(\varphi_{0}(z)+\frac{a}{s^{1 / 2}}(1+i \delta)\right) \text { with, } F(u)=(1+i \delta)|u|^{p-1} u
\end{aligned}
$$

Since $|z|<1$, there exists positive $c_{0}$ and $s_{0}$ such that $\left|\varphi_{0}(z)\right|$ and $\left|\varphi_{0}(z)+\frac{a}{s^{1 / 2}}(1+i \delta)\right|$ are both larger that $\frac{1}{c_{0}}$ ans smaller than $c_{0}$, uniformly in $|z|<1$ and $s>s_{0}$. Since $F(u)$ is $C^{\infty}$ for $\frac{1}{c_{0}} \leq|u| \leq c_{0}$, we expand it around $u=\varphi_{0}(z)$ as follows

$$
\left|F\left(\varphi_{0}(z)+\frac{a}{s^{1 / 2}}(1+i \delta)\right)-F\left(\varphi_{0}(z)\right)-\sum_{j=1}^{n} \frac{1}{s^{j / 2}} F_{j}\left(\varphi_{0}(z)\right)\right| \leq C \frac{1}{s^{\frac{n+1}{2}}},
$$

where $F_{j}(u)$ are $C^{\infty}$. Hence, we can expand $F_{j}(u)$ around $u=\varphi_{0}(0)$ and write

$$
\left|F\left(\varphi_{0}(z)+\frac{a}{s^{1 / 2}}(1+i \delta)\right)-F\left(\varphi_{0}(z)\right)-\sum_{j=1}^{n} \sum_{l=0}^{n-j} \frac{c_{j, l}}{s^{j / 2}} z^{2 l}\right| \leq \sum_{j=1}^{n} \frac{C}{s^{\frac{j}{2}}}|z|^{2(n-j)+2}+\frac{C}{s^{\frac{n+1}{2}}},
$$

Similarly, we have the following

$$
\begin{gathered}
\left|\frac{z}{s} \nabla_{z} \varphi_{0}(z)-\frac{|z|^{2}}{s} \sum_{j=0}^{n-2} d_{j} z^{2 j}\right| \leq \frac{C}{s}|z|^{2 n} \\
\left|\frac{1}{s^{1 / 2}} \Delta_{z} \varphi_{0}(z)-\frac{1}{s^{1 / 2}} \sum_{j=0}^{n-1} b_{j} z^{2 j}\right| \leq \frac{C}{s^{1 / 2}}|z|^{2 n} \text { and }\left|\varphi_{0}(z)-\sum_{j=0}^{n-1} e_{j} z^{2 j}\right| \leq C|z|^{2 n} .
\end{gathered}
$$

Recalling that $z=\frac{y}{s^{1 / 4}}$, we get the conclusion of the Lemma.

In the following, we introduce $F_{j}\left(R^{*}\right)(\theta, s)$ as the projection of the rest term $R^{*}\left(\theta^{\prime}, y, s\right)$ on the standard Hermite polynomial, introduced in (21). 
Lemma 4.19 (Projection of $R^{*}$ on the eigenfunctions of $\mathcal{L}$ ) It holds that $F_{j}\left(R^{*}\right)\left(\theta^{\prime}, s\right) \equiv$ 0 when $j$ is odd, and $\left|F_{j}\left(R^{*}\right)\left(\theta^{\prime}, s\right)\right| \leq C \frac{1+s\left|\theta^{\prime}(s)\right|}{s^{\frac{j}{4}+\frac{1}{2}}}$, when $j$ is even and $j \geq 4$.

$$
\begin{aligned}
F_{0}\left(R^{*}\right)\left(\theta^{\prime}, s\right)= & -i \theta^{\prime}(s)\left(\kappa+O\left(\frac{1}{s^{1 / 2}}\right)\right)+(1+i \delta)\left\{-2 \frac{\kappa b}{(p-1)^{2}}+a\right\} \frac{1}{s^{1 / 2}}+\frac{a\left(p-\delta^{2}\right)}{\kappa} \frac{1}{s} \\
& +i \kappa \delta\left\{\frac{a^{2}}{\kappa^{2}}(1+p)-\frac{\mu}{\delta}+12 \frac{b^{2}(p+1)}{(p-1)^{4}}-\frac{4 a b(p+1)}{\kappa(p-1)^{2}}+O\left(\frac{1}{s^{1 / 2}}\right)\right\} \frac{1}{s}+O\left(\frac{1}{s^{3 / 2}}\right)
\end{aligned}
$$

with the fact that $\delta^{2}=p$, we obtain

$$
\begin{aligned}
F_{0}\left(R^{*}\right)\left(\theta^{\prime}, s\right)= & -i \theta^{\prime}(s)\left(\kappa+O\left(\frac{1}{s^{1 / 2}}\right)\right)+(1+i \delta)\left\{-2 \frac{\kappa b}{(p-1)^{2}}+a\right\} \frac{1}{s^{1 / 2}} \\
& +i \kappa \delta\left\{\frac{a^{2}}{\kappa^{2}}(1+p)-\frac{\mu}{\delta}+12 \frac{b^{2}(p+1)}{(p-1)^{4}}-\frac{4 a b(p+1)}{\kappa(p-1)^{2}}+O\left(\frac{1}{s^{1 / 2}}\right)\right\} \frac{1}{s}+O\left(\frac{1}{s^{3 / 2}}\right)
\end{aligned}
$$

If $j=2$, then

$$
\begin{aligned}
\left.F_{2}\left(R^{*}\right)\left(\theta^{\prime}, s\right)\right)= & i \theta^{\prime}(s)\left(\frac{\kappa b}{(p-1)^{2}}(1+i \delta) \frac{1}{s^{1 / 2}}-6 \kappa \frac{b^{2}\left(p-\delta^{2}\right)}{(p-1)^{4}} \frac{1}{s}-i 6 \kappa \frac{b^{2} \delta(p+1)}{(p-1)^{4}} \frac{1}{s}+O\left(\frac{1}{s^{3 / 2}}\right)\right) \\
& \left(6 \kappa \frac{b^{2}\left(p-\delta^{2}\right)}{(p-1)^{4}}-\frac{2 a b\left(p-\delta^{2}\right)}{(p-1)^{2}}\right) \frac{1}{s}+i\left(6 \kappa \frac{\delta b^{2}(p+1)}{(p-1)^{4}}-\frac{2 a b \delta(p+1)}{(p-1)^{2}}\right) \frac{1}{s} \\
& +\frac{\kappa b}{(p-1)^{2}}\left\{-\left(\frac{1}{2}+\mu \delta\right)+p(p+1)\left(\frac{a^{2}}{\kappa^{2}}+60 \frac{b^{2}}{(p-1)^{4}}-12 \frac{a b^{2}}{(p-1)^{4}}\right)\right\} \frac{1}{s^{3 / 2}}+i O\left(\frac{1}{s^{3 / 2}}\right)+O\left(\frac{1}{s^{2}}\right) .
\end{aligned}
$$

Then, by the fact that $\delta^{2}=p$,

$$
\begin{aligned}
\left.F_{2}\left(R^{*}\right)\left(\theta^{\prime}, s\right)\right)= & i \theta^{\prime}(s)\left(\frac{\kappa b}{(p-1)^{2}}(1+i \delta) \frac{1}{s^{1 / 2}}-i 6 \kappa \frac{b^{2} \delta(p+1)}{(p-1)^{4}} \frac{1}{s}+O\left(\frac{1}{s^{3 / 2}}\right)\right) \\
& +i\left(6 \kappa \frac{\delta b^{2}(p+1)}{(p-1)^{4}}-\frac{2 a b \delta(p+1)}{(p-1)^{2}}\right) \frac{1}{s}+i O\left(\frac{1}{s^{3 / 2}}\right) \\
& +\frac{\kappa b}{(p-1)^{2}}\left\{-\left(\frac{1}{2}+\mu \delta\right)+p(p+1)\left(\frac{a^{2}}{\kappa^{2}}+60 \frac{b^{2}}{(p-1)^{4}}-12 \frac{a b^{2}}{(p-1)^{4}}\right)\right\} \frac{1}{s^{3 / 2}}+O\left(\frac{1}{s^{2}}\right) .
\end{aligned}
$$

Proof: Since $R^{*}$ is even in the $y$ variables and $f_{j}$ is odd when $j$ is odd, $F_{j}\left(R^{*}\right)\left(\theta^{\prime}, s\right) \equiv 0$, when $j$ is odd.

Now, when $j$ is even, we apply Lemma 4.18 with $n=\left[\frac{j}{2}\right]$ and write

$$
R^{*}\left(\theta^{\prime}, y, s\right)=\Pi_{\frac{j}{2}}\left(\theta^{\prime}, y, s\right)+O\left(\frac{1+s\left|\theta^{\prime}(s)\right|+|y|^{j}}{s \frac{j}{4}+\frac{1}{2}}\right),
$$

where $\Pi_{\frac{j}{2}}$ is a polynomials in $y$ of degree less than $j-1$. Using the definition of $F_{j}\left(R^{*}\right)$ (projection on the $h_{j}$ of $R^{*}$ ), we write

$$
\begin{aligned}
& \int_{\mathbb{R}^{N}} R^{*} h_{j} \rho=\int_{|y|<s^{1 / 4}} R^{*} h_{j} \rho d y+\int_{|y|>s^{1 / 4}} R^{*} h_{j} \rho d y \\
& =\int_{|y|<s^{1 / 4}} \Pi_{\frac{j}{2}} h_{j} \rho d y+O\left(\int_{|y|<s^{1 / 4}} \frac{1+s\left|\theta^{\prime}(s)\right|+|y|^{j}}{s \frac{j}{4}+\frac{1}{2}} h_{j} \rho d y\right)+\int_{|y|>s^{1 / 4}} R^{*} h_{j} \rho d y \\
& =\int_{\mathbb{R}^{N}} \Pi_{\frac{j}{2}} h_{j} \rho d y+O\left(\frac{1+s\left|\theta^{\prime}(s)\right|}{s \frac{j}{4}+\frac{1}{2}}\right)+\int_{|y|>s^{1 / 4}} R^{*} h_{j} \rho d y+\int_{|y|>s^{1 / 4}} \Pi_{\frac{j}{2}} h_{j} \rho d y
\end{aligned}
$$


We can see that $\int_{\mathbb{R}^{N}} \Pi_{\frac{j}{2}} h_{j} \rho d y=0$ because $h_{j}$ is orthogonal to all polynomials of degree less than $j-1$. Then, note that both integrals over the domain $\left\{|y|>s^{1 / 4}\right\}$ are controlled by

$$
\int_{s^{1 / 4}}\left(\left|R^{*}\left(\theta^{\prime}, y, s\right)\right|+1+|y|^{j}\right)\left(1+|y|^{j}\right) \rho d y .
$$

Using the fact that $R(y, s)$ measures the defect of $\varphi(y, s)$ from being an exact solution of (14). However, since $\varphi$ is an approximate solution of (14), one easily derive the fact that

$$
\begin{aligned}
& \|R(s)\|_{L^{\infty}} \leq \frac{C}{\sqrt{s}}, \text { and } \\
& \left|R^{*}\left(\theta^{\prime}, y, s\right)\right| \leq \frac{C}{\sqrt{s}}+\left|\theta^{\prime}(s)\right| .
\end{aligned}
$$

Using the fact that $|\rho(y)| \leq C e^{\sqrt{-s}}$, for $|y|>s^{1 / 4}$, we can bound our integral by

$$
C\left(1+\left|\theta^{\prime}(s)\right|\right) \int_{\mathbb{R}^{N}}\left(1+|y|^{j}\right)^{2} e^{c \sqrt{-s}} \sqrt{\rho} d y=C(j)\left(1+\left|\theta^{\prime}(s)\right|\right) e^{c \sqrt{-s}} .
$$

This inequality gives us the result for $j \geq 4$.

If $j=0$ or $j=2$, one has to refine Lemma 4.18 in straightforward but long way and do as we did for general $j$. The details are given in Appendix C. This concludes the proof of the lemma.

Corollary 4.4 Projection of $R^{*}$ on the eigenfunctions of $\tilde{\mathcal{L}}$

If $j$ is even and $j \geq 4$, then $\tilde{P}_{j}\left(R^{*}\right)\left(\theta^{\prime}, s\right)$ and $\hat{P}_{j}\left(R^{*}\right)\left(\theta^{\prime}, s\right)$ are $O\left(\frac{1+s\left|\theta^{\prime}\right|}{s^{\frac{j}{4}+\frac{1}{2}}}\right)$.

If $j$ is odd, then $\tilde{P}_{j}\left(R^{*}\right)\left(\theta^{\prime}, s\right)=\hat{P}_{j}\left(R^{*}\right)\left(\theta^{\prime}, s\right)=0$.

If $j=0$, then,

$$
\begin{aligned}
\hat{P}_{0}\left(R^{*}\right)\left(\theta^{\prime}, s\right)= & -\theta^{\prime}(s)\left(\kappa+O\left(\frac{1}{s^{1 / 2}}\right)\right) \\
& +\kappa \delta\left\{\frac{a^{2}}{\kappa^{2}} \delta(1+p)-\mu \kappa+12 \frac{b^{2}(p+1)}{(p-1)^{4}}-\frac{4 a b(p+1)}{\kappa(p-1)^{2}}\right\} \frac{1}{s}+O\left(\frac{1}{s^{3 / 2}}\right)
\end{aligned}
$$

and

$$
\tilde{P}_{0}\left(R^{*}\right)\left(\theta^{\prime}, s\right)=O\left(\frac{\theta^{\prime}(s)}{s^{1 / 2}}\right)+\left\{-2 \frac{\kappa b}{(p-1)^{2}}+a\right\} \frac{1}{s^{1 / 2}}+O\left(\frac{1}{s^{3 / 2}}\right) .
$$

If $j=2$, then $\hat{P}_{2}\left(R^{*}\right)\left(\theta^{\prime}, s\right)=O\left(\frac{1}{s}\right)+O\left(\frac{\theta^{\prime}(s)}{s^{1 / 2}}\right)$ and

$$
\begin{aligned}
\tilde{P}_{2}\left(R^{*}\right)= & \theta^{\prime}(s)\left(-\frac{\kappa b \delta}{(p-1)^{2}} \frac{1}{s^{1 / 2}}+O\left(\frac{1}{s}\right)\right) \\
& +\frac{\kappa b}{(p-1)^{2}}\left\{-\frac{1}{2}-\mu \delta+p(p+1)\left(\frac{a^{2}}{\kappa^{2}}+60 \frac{b^{2}}{(p-1)^{4}}-12 \frac{a b^{2}}{(p-1)^{4}}\right)\right\} \frac{1}{s^{3 / 2}}+O\left(\frac{1}{s^{2}}\right) .
\end{aligned}
$$

In the following, we give a new version of Corollary 4.4. 
Corollary 4.5 If we choose $a, b$ and $\mu$ as follows

$$
\begin{aligned}
& a=\frac{2 \kappa b}{(p-1)^{2}}, \\
& \mu=8 \delta(p+1) \frac{b^{2}}{(p-1)^{4}}, \\
& \left(\frac{1}{2}+\mu \delta\right)=p(p+1)\left(\frac{a^{2}}{\kappa^{2}}+60 \frac{b^{2}}{(p-1)^{4}}-12 \frac{a b^{2}}{(p-1)^{4}}\right),
\end{aligned}
$$

If $j$ is even and $j \geq 4$, then $\tilde{P}_{j}\left(R^{*}\right)\left(\theta^{\prime}, s\right)$ and $\hat{P}_{j}\left(R^{*}\right)\left(\theta^{\prime}, s\right)$ are $O\left(\frac{1+s\left|\theta^{\prime}\right|}{s^{\frac{j}{4}+\frac{1}{2}}}\right)$.

If $j$ is odd, then $\tilde{P}_{j}\left(R^{*}\right)\left(\theta^{\prime}, s\right)=\hat{P}_{j}\left(R^{*}\right)\left(\theta^{\prime}, s\right)=0$.

If $j=0$, then,

$$
\hat{P}_{0}\left(R^{*}\right)\left(\theta^{\prime}, s\right)=-\theta^{\prime}(s)\left(\kappa+O\left(\frac{1}{s^{1 / 2}}\right)\right)+O\left(\frac{1}{s^{3 / 2}}\right)
$$

and

$$
\tilde{P}_{0}\left(R^{*}\right)\left(\theta^{\prime}, s\right)=O\left(\frac{\theta^{\prime}(s)}{s^{1 / 2}}\right)+O\left(\frac{1}{s^{3 / 2}}\right) .
$$

If $j=2$, then $\hat{P}_{2}\left(R^{*}\right)\left(\theta^{\prime}, s\right)=O\left(\frac{1}{s}\right)+O\left(\frac{\theta^{\prime}(s)}{s^{1 / 2}}\right)$ and

$$
\tilde{P}_{2}\left(R^{*}\right)=\theta^{\prime}(s)\left(-\frac{\kappa b \delta}{(p-1)^{2}} \frac{1}{s^{1 / 2}}+O\left(\frac{1}{s}\right)\right)+O\left(\frac{1}{s^{2}}\right)
$$

Remark 4.20 It is very important to note that $a, b$ and $\mu$ chosen by (106) are the same given by our formal approach (See Section Q 2 ).

\section{Part 2: Proof of Proposition 4.10}

In this part, we consider $A \geq 1$ and take $s$ large enough so that Part 1 applies.

(i) We control $\theta^{\prime}(s)$, from the projection of (42) on $\hat{h}_{0}=i h_{0}$, taking on consideration the modulation, we obtain

$$
\hat{q}_{0}^{\prime}=-\left(\frac{\mu}{s}+\theta^{\prime}\right)\left[\left(1+\delta^{2}\right) \tilde{q}_{0}+\delta \hat{q}_{0}\right]+\hat{P}_{0}\left(V_{1} q\right)+\hat{P}_{0}\left(V_{2} \bar{q}\right)+\hat{P}_{0}(B)+\hat{P}_{0}\left(R^{*}\right)
$$

By the definition of the shrinking set $\mathcal{V}_{A}$ Definition 4.3, Corollary 4.3, Lemma 4.17 and Corollary [4.5, since $q(s) \in \mathcal{V}_{A}(s)$ and $\hat{q}_{0}=0$ for all $s \in\left[\tau, s_{1}\right]$, this yields

$$
\left|\theta^{\prime}(s)\right| \leq \frac{C A^{5}}{s^{\frac{3}{2}}} .
$$

(ii) From the projection of (42) respectively on $\tilde{h}_{0}=(1+i \delta) h_{0}$ and $\tilde{h}_{1}=(1+i \delta) h_{1}$, we obtain;

$$
\begin{aligned}
& \tilde{q}_{0}^{\prime}=\tilde{q}_{0}+\left(\frac{\mu}{s}+\theta^{\prime}\right)\left[\delta \tilde{q}_{0}+\hat{q}_{0}\right]+\tilde{P}_{0}\left(V_{1} q\right)+\tilde{P}_{0}\left(V_{2} \bar{q}\right)+\tilde{P}_{0}(B)+\tilde{P}_{0}\left(R^{*}\right), \\
& \tilde{q}_{1}^{\prime}=\frac{1}{2} \tilde{q}_{1}+\left(\frac{\mu}{s}+\theta^{\prime}\right)\left[\delta \tilde{q}_{1}+\hat{q}_{1}\right]+\tilde{P}_{1}\left(V_{1} q\right)+\tilde{P}_{1}\left(V_{2} \bar{q}\right)+\tilde{P}_{1}(B)+\tilde{P}_{1}\left(R^{*}\right) .
\end{aligned}
$$


The two inequalities in (ii) of Proposition 4.10 are a direct consequence of Definition 4.3. Remark 4.15, Lemma 4.17 and Corollary 4.5, provided that $s_{0}$ is large enough.

(iii) Estimate of $\tilde{q}_{2}$. By Corollary 4.3, Corollary 4.4, equation (107) and the fact that $q(s) \in \mathcal{V}_{A}(s)$, we obtain

$$
\left|\theta^{\prime} \kappa-16 \delta \frac{b(p+1)}{(p-1)^{2}} \frac{\tilde{q}_{2}}{s^{1 / 2}}\right| \leq \frac{C A^{3}}{s^{\frac{3}{2}}}
$$

Let us project the different terms of (42) on $\tilde{h}_{2}$. We use Part 1, (Lemma 4.14 and Corollary 4.4) and the fact that $q(s) \in \mathcal{V}_{A}(s)$ and we obtain:

$$
\begin{aligned}
& \tilde{P}_{2}\left(\frac{\partial q}{\partial s}\right)=\tilde{q}_{2}^{\prime}, \\
& \mid \tilde{P}_{2}\left(-i\left(\frac{\mu}{s}+\theta^{\prime}(s) q\right)-\mu \delta \frac{\tilde{q}_{2}}{s} \mid \leq C \frac{A^{3}}{s^{2}},\right. \\
& \left|\tilde{P}_{2}\left(V_{1} q\right)+\frac{\tilde{q}_{2}}{s} 60 b^{2}(p+1) \frac{1}{(p-1)^{2}}+\tilde{P}_{2}\left(V_{2} \bar{q}\right)-\frac{\tilde{q}_{2}}{s} 60 b^{2}(p+1) \frac{p^{2}-4 p+1}{2(p-1)^{3}}\right| \leq C \frac{A^{3}}{s^{2}} \\
& \left|\tilde{P}_{2}(B(q, y, s))\right|+\left|\hat{P}_{n}(B(q, y, s))\right| \leq \frac{C}{s^{2}},
\end{aligned}
$$

and

$$
\left|\tilde{P}_{2}\left(R^{*}(q, y, s)\right)+16 \frac{b^{2}}{(p-1)^{4}} p(p+1) \frac{\tilde{q}_{2}}{s}\right| \leq \frac{C A^{3}}{s^{2}},
$$

here we used (108), the estimate on $\theta^{\prime}$ and Corollary 4.5.

Adding all these contributions gives -2 as the coefficient of $\frac{\tilde{q}_{2}(s)}{s}$ in the following ODE

$$
\left|\tilde{q}_{2}^{\prime}+\frac{2}{s} \tilde{q}_{2}\right| \leq \frac{C A^{3}}{s^{2}}
$$

(iv) Estimates of $\hat{q}_{1}, \hat{q}_{2}, \hat{q}_{j}$ and $\tilde{q}_{j}$ for $3 \leq j \leq M$ : Using the definintion of the shrinking set $\mathcal{V}_{A}$ Definition 4.3 and Corollary 4.3. Lemma 4.17, Corollary 4.4 from Part 1 and the fact that $q(s) \in \mathcal{V}_{A}(s)$, we see that for all $s \in\left[\tau, s_{1}\right]$, we have

$$
\mid \begin{array}{lll}
\hat{q}_{1}^{\prime}+\frac{1}{2} \hat{q}_{1} \mid \leq C \frac{A^{5}}{s^{\frac{3}{2}}}, & \left|\hat{q}_{2}^{\prime}+\hat{q}_{2}\right| \leq \frac{C}{s}, & \\
\hat{q}_{j}^{\prime}+\frac{j}{2} \hat{q}_{j} \mid \leq C \frac{A^{j-1}}{s^{\frac{j+1}{4}}}, & \text { if } & 3 \leq j \leq M, \\
\tilde{q}_{j}^{\prime}+\frac{j-2}{2} \tilde{q}_{j} \mid \leq C \frac{A^{j-1}}{s^{\frac{j+1}{4}}}, & \text { if } & 3 \leq j \leq M .
\end{array}
$$

Integrating this inequalities between $\tau$ and $s_{1}$ gives the desired estimates.

\subsubsection{The infinite dimensional part: $q_{-}$}

We proceed in 2 parts:

- In Part 1, we project equation (42) to get equations satisfied by $q_{-}$.

- In Part 2, we prove the estimate on $q_{-}$. 
Part 1: Projection of equation (42) using the projector $P_{-}$In the following, we will project equation (42) term by term.

First term: $\frac{\partial q}{\partial s}$

From (52), its projection is

$$
P_{-}\left(\frac{\partial q}{\partial s}\right)=\frac{\partial q_{-}}{\partial s}
$$

Second term: $\tilde{\mathcal{L}}_{0} q$

From (42), we have the following,

$$
P_{-}(\tilde{\mathcal{L}} q)=\mathcal{L}_{0} q_{-}+P_{-}\left[(1+i \delta) \operatorname{Re} q_{-}\right] .
$$

Third term: $-i\left(\frac{\mu}{s}+\theta^{\prime}(s)\right) q$

Since $P_{-}$commutes with the multiplication by $i$, we deduce that

$$
P_{-}\left[-i\left(\frac{\mu}{s}+\theta^{\prime}(s)\right) q\right]=-i\left(\frac{\mu}{s}+\theta^{\prime}(s)\right) q_{-} .
$$

Fourth term: $V_{1} q$ and $V_{2} \bar{q}$

We have the following:

Lemma 4.21 (Projection of $V_{1} q$ and $V_{2} \bar{q}$ ) The projection of $V_{1} q$ and $V_{2} \bar{q}$ satisfy for all $s \geq 1$,

$$
\left\|\frac{P_{-}\left(V_{1} q\right)}{1+|y|^{M+1}}\right\|_{L^{\infty}} \leq\left(\left\|V_{1}\right\|_{L^{\infty}}+\frac{C}{s^{1 / 2}}\right)\left\|\frac{q_{-}}{1+|y|^{M+1}}\right\|_{L^{\infty}}+\sum_{n=0}^{M} \frac{C}{s^{\frac{M+1-n}{4}}}\left(\left|\hat{q}_{n}\right|+\left|\tilde{q}_{n}\right|\right),
$$

and the same holds for $V_{2} \bar{q}$.

Using the fact that $q(s) \in \mathcal{V}_{A}(s)$, we get the following

Corollary 4.6 For all $A \geq 1$, there exists $s_{14}(A)$ such that for all $s \geq s_{14}$, if $q(s) \in \mathcal{V}_{A}(s)$ then,

$$
\left\|\frac{P_{-}\left(V_{1} q\right)}{1+|y|^{M+1}}\right\|_{L^{\infty}} \leq\left\|V_{1}\right\|_{L^{\infty}}\left\|\frac{q_{-}}{1+|y|^{M+1}}\right\|_{L^{\infty}}+C \frac{A^{M}}{s^{\frac{M+2}{4}}}
$$

and the same holds for $V_{2} q$.

Proof of Lemma 4.21: We just give the proof for $V_{1} q$ since the proof for $V_{2} \bar{q}$ is similar.

From Subsection 3.3, we write $q=q_{+}+q_{-}$and

$$
P_{-}\left(V_{1} q\right)=V_{1} q-P_{+}\left(V_{1} q_{-}\right)+P_{-}\left(V_{1} q_{+}\right) .
$$

Moreover, we claim that the following estimates hold

$$
\left\|\frac{V_{1} q_{-}}{1+|y|^{M+1}}\right\|_{L^{\infty}} \leq\left\|V_{1}\right\|_{L^{\infty}}\left\|\frac{q_{-}}{1+|y|^{M+1}}\right\|_{L^{\infty}}
$$

Indeed, the first one is obvious. To prove the second one, we use (76) to show that 


$$
\left|\hat{P}_{n}\left(V_{1} q_{-}\right)\right|+\left|\tilde{P}_{n}\left(V_{1} q_{-}\right)\right| \leq \frac{C}{s^{1 / 2}}\left\|\frac{q_{-}}{1+|y|^{M+1}}\right\|_{L^{\infty}} .
$$

To control $P_{-}\left(V_{1} q_{+}\right)=\sum_{n \leq M} P_{-}\left(V_{1}\left(\hat{q}_{n} \hat{h}_{n}+\tilde{q}_{n} \tilde{h}_{n}\right)\right)$, we argue as follows.

If $M-n$ is odd, we take $k=\frac{M-1-n}{2}$ in (77), hence

$$
P_{-}\left(V_{1}\left(\hat{q}_{n} \hat{h}_{n}+\tilde{q}_{n} \tilde{h}_{n}\right)\right)=\sum_{j=1}^{k} \frac{1}{s^{\frac{j}{2}}} P_{-}\left(W_{1, j}\left(\hat{q}_{n} \hat{h}_{n}+\tilde{q}_{n} \tilde{h}_{n}\right)\right)+P_{-}\left(\left(\hat{q}_{n} \hat{h}_{n}+\tilde{q}_{n} \tilde{h}_{n}\right) \tilde{W}_{1, k}\right)
$$

Since $2 k+n \leq M$, we deduce that $P_{-}\left(W_{1, j}\left(\hat{q}_{n} \hat{h}_{n}+\tilde{q}_{n} \tilde{h}_{n}\right)\right)=0$ for all $0 \leq j \leq k$. Moreover, using that

$$
\left|\tilde{W}_{1, k}\right| \leq C \frac{\left(1+|y|^{2 k+2}\right)}{s^{\frac{k+1}{2}}}
$$

and applying Lemma A.3, we deduce that

$$
\left\|\frac{P_{-}\left(V_{1}\left(\hat{q}_{n} \hat{h}_{n}+\tilde{q}_{n} \tilde{h}_{n}\right)\right)}{1+|y|^{M+1}}\right\|_{L^{\infty}} \leq C \frac{\left|\hat{q}_{n}\right|+\left|\tilde{q}_{n}\right|}{s^{\frac{M+1-n}{4}}} .
$$

If $M-n$ is even, we take $k=\frac{M-n}{2}$ in (77) and use that

$$
\left|\tilde{W}_{1, k}\right| \leq C \frac{1+|y|^{2 k+1}}{s^{\frac{k}{2}+\frac{1}{4}}}
$$

to deduce that (112) holds. This ends the proof of Lemma 4.21,

Fifth term: $B(q, y, s)$.

Using (44), we have the following estimate from Lemmas A.3 and 4.16.

Lemma 4.22 For all $K \geq 1$ and $A \geq 1$, there exists $s_{15}(K, A)$ such that for all $s \geq s_{15}$, if $q(s) \in \mathcal{V}_{A}(s)$, then

$$
\left\|\frac{P_{-}(B(q, y, s))}{1+|y|^{M+1}}\right\|_{L^{\infty}} \leq C(M)\left[\left(\frac{A^{M+2}}{s^{\frac{1}{4}}}\right)^{\bar{p}}+\frac{A^{5}}{s^{\frac{1}{2}}}\right] \frac{1}{s^{\frac{M+1}{4}}},
$$

where $\bar{p}=\min (p, 2)$.

Proof: The proof is very similar to the proof of the previous lemma. From Lemma 4.16, we deduce that for all $s$ there exists a polynomial $B_{M}$ of degree $M$ in $y$ such that for all $y$ and $s$, we have

$$
\left|B-B_{M}(y)\right| \leq C\left[\left(\frac{A^{M+2}}{s^{\frac{1}{4}}}\right)^{\bar{p}}+\frac{A^{\left[5+(M+1)^{2}\right]}}{s^{\frac{1}{2}}}\right] \frac{\left(1+|y|^{M+1}\right)}{s^{\frac{M+1}{4}}} .
$$

Indeed, we can take $B_{M}$ to be the polynomial

$$
B_{M}=P_{+, M}\left[\sum_{\substack{l=0 \\ 0 \leq j, k \leq M+1 \\ 2 \leq j+k \leq M+1}} \frac{1}{s^{l / 2}}\left[B_{j, k}^{l}\left(\frac{y}{s^{\frac{1}{4}}}\right) q_{+}^{j} \bar{q}_{+}^{k}\right]\right] .
$$


Then the fact that $B-B_{M}(y)$ is controlled by the right hand side of (114) is a consequence of the following estimates in the outer region and in the inner region.

First, in the region $|y| \geq s^{\frac{1}{4}}$, we have from Lemma 4.16,

$$
|B| \leq C|q|^{\bar{p}} \leq C\left(\frac{A^{M+2}}{s^{\frac{1}{4}}}\right)^{\bar{p}}
$$

and from the proof of Lemma 4.17, we know that for $0 \leq n \leq M$,

$$
\left|\tilde{P}_{n}\left(B_{M}(q, y, s)\right)\right|+\left|\hat{P}_{n}\left(B_{M}(q, y, s)\right)\right| \leq C \frac{A^{n}}{s^{\frac{n+2}{4}}} .
$$

Beside, in the region $|y| \leq s^{\frac{1}{4}}$, we can use the same argument as in the proof of Lemma 4.16 to deduce that the coefficients of degree $k \geq M+1$ of the polynomial

$$
\sum_{\substack { l=0 \\
\begin{subarray}{c}{0 \leq j, k \leq M+1 \\
2 \leq j+k \leq M+1{ l = 0 \\
\begin{subarray} { c } { 0 \leq j , k \leq M + 1 \\
2 \leq j + k \leq M + 1 } }\end{subarray}}^{\sum^{l / 2}}\left[B_{j, k}^{l}\left(\frac{y}{s^{\frac{1}{4}}}\right) q_{+}^{j} \bar{q}_{+}^{k}\right]-B_{M},
$$

is controlled by $C \frac{A^{k}}{s^{\frac{k}{4}+\frac{1}{2}}}$ and hence

$$
\left|\sum_{\substack{l=0 \\ 0 \leq j, k \leq M+1 \\ 2 \leq j+k \leq M+1}}^{M} \frac{1}{s^{l / 2}}\left[B_{j, k}^{l}\left(\frac{y}{s^{\frac{1}{4}}}\right) q_{+}^{j} \bar{q}_{+}^{k}\right]-B_{M}\right| \leq C \frac{A^{2 M+2}}{s^{\frac{M+3}{4}}}\left(1+|y|^{M+1}\right),
$$

in the region $|y| \leq s^{\frac{1}{4}}$.

Moreover, using that $|q| \leq C \frac{A^{M+1}}{s^{\frac{1}{4}}}$ in the region $|y| \leq s^{\frac{1}{4}}$, we deduce that for all $s \geq 2 A^{2}$, we have

$$
\left|\sum_{\substack { l=0 \\
\begin{subarray}{c}{0 \leq j, k \leq M+1 \\
2 \leq j+k \leq M+1{ l = 0 \\
\begin{subarray} { c } { 0 \leq j , k \leq M + 1 \\
2 \leq j + k \leq M + 1 } }\end{subarray}}^{M} \frac{1}{s^{l / 2}}\left[B_{j, k}^{l}\left(\frac{y}{s^{\frac{1}{4}}}\right) q_{+}^{j} \bar{q}_{+}^{k}\right]\right| \leq C \frac{A^{2 M+2}}{s^{\frac{M+3}{4}}}\left(1+|y|^{M+1}\right) .
$$

Finally, to control the term $|q|^{M+2}$, we use the fact that in the region $|y| \leq s^{\frac{1}{4}}$, we have the following two estimates $|q| \leq C \frac{A^{M+1}}{s^{\frac{1}{4}}}$ and $|q| \leq A^{5} \frac{1}{s^{\frac{3}{4}}}\left(1+|y|^{M+1}\right)$ if $\sqrt{s} \geq 2 A^{2}$. Hence

$$
|q|^{M+2} \leq C \frac{A^{5}}{s^{\frac{3}{4}}}\left(\frac{A^{M+1}}{s^{\frac{1}{4}}}\right)^{M+1}\left(1+|y|^{M+1}\right) .
$$

This ends the proof of estimate (114) and conclude the proof of (113) by applying Lemma A.3.

Sixth term: $R^{*}\left(\theta^{\prime}, y, s\right)$.

We claim the following: 
Lemma 4.23 If $\left|\theta^{\prime}(s)\right| \leq \frac{C A^{5}}{s^{3 / 2}}$, then the following holds

$$
\left\|\frac{P_{-}\left(R^{*}\left(\theta^{\prime}, y, s\right)\right)}{1+|y|^{M+1}}\right\| \leq C \frac{1}{s^{\frac{M+3}{4}}}
$$

Proof: Taking $n=\frac{M}{2}+1$ (remember $M$ is even), we write from Lemma 4.18 $R^{*}\left(\theta^{\prime}, y, s\right)=$ $\Pi_{n}\left(\theta^{\prime}, y, s\right)+\tilde{\Pi}_{n}\left(\theta^{\prime}, y, s\right)$. Since $2 n-2=M$, we see from subsection 3.3 that

$$
\left|\tilde{\Pi}_{n}\left(\theta^{\prime}, y, s\right)\right| \leq C \frac{1+|y|^{2 n-2}}{s^{\frac{n+1}{2}}} \leq C \frac{1+|y|^{M+1}}{s^{\frac{M+3}{4}}}
$$

in the region $|y|<s^{1 / 4}$. It is easy to see using 46 and the definition of $\Pi_{n}$ that (115) holds for all $y \in \mathbb{R}$ and $s \geq 1$. Then applying Lemma A.3, we conclude easily.

Part 2: Proof of the last but one identity in (iv) of Proposition 4.10 (estimate on $q_{-}$)

If we apply the projection $P_{-}$to the equation (42) satisfied by $q$, we see that $q_{-}$satisfies the following equation:

$$
\frac{\partial q_{-}}{\partial s}=\mathcal{L}_{0} q_{-}+P_{-}\left[(1+i \delta) \operatorname{Re} q_{-}\right]+P_{-}\left[-i\left(\frac{\mu}{s}+\theta^{\prime}(s)\right) q+V_{1} q+V_{2} \bar{q}+B(q, y, s)+R^{*}\left(\theta^{\prime}, y, s\right)\right]
$$

Here, we have used the important fact that $P_{-}\left[(1+i \delta) \operatorname{Re} q_{+}\right]=0$. The fact that $M$ is large as fixed in (50) is crucial in the proof. Using the kernel of the semigroup generated by $\mathcal{L}_{0}$, we get for all $s \in\left[\tau, s_{1}\right]$,

$$
\begin{aligned}
q_{-}(s)= & e^{(s-\tau) \mathcal{L}_{0}} q_{-}(\tau) \\
& +\int_{\tau}^{s} e^{\left(s-s^{\prime}\right) \mathcal{L}_{0}} P_{-}\left[(1+i \delta) \operatorname{Re} q_{-}\right] d s^{\prime} \\
& +\int_{\tau}^{s} e^{\left(s-s^{\prime}\right) \mathcal{L}_{0}} P_{-}\left[-i\left(\frac{\mu}{s}+\theta^{\prime}\left(s^{\prime}\right)\right) q+V_{1} q+V_{2} \bar{q}+B\left(q, y, s^{\prime}\right)+R^{*}\left(\theta^{\prime}, y, s^{\prime}\right)\right] d s^{\prime} .
\end{aligned}
$$

Using Lemma A.2, we get

$$
\begin{aligned}
& \left\|\frac{q_{-}(s)}{1+|y|^{M+1}}\right\|_{L^{\infty}} \leq e^{-\frac{M+1}{2}(s-\tau)}\left\|\frac{q_{-}(\tau)}{1+|y|^{M+1}}\right\|_{L^{\infty}} \\
& +\int_{\tau}^{s} e^{-\frac{M+1}{2}\left(s-s^{\prime}\right)} \sqrt{1+\delta^{2}}\left\|\frac{q_{-}}{1+|y|^{M+1}}\right\|_{L^{\infty}} d s^{\prime} \\
& +\int_{\tau}^{s} e^{-\frac{M+1}{2}\left(s-s^{\prime}\right)}\left\|\frac{P_{-}\left[-i\left(\frac{\mu}{s}+\theta^{\prime}\left(s^{\prime}\right)\right) q+V_{1} q+V_{2} \bar{q}+B\left(q, y, s^{\prime}\right)+R^{*}\left(\theta^{\prime}, y, s^{\prime}\right)\right]}{1+|y|^{M+1}}\right\|_{L^{\infty}} d s^{\prime}
\end{aligned}
$$

Assuming that $q\left(s^{\prime}\right) \in \mathcal{V}_{A}\left(s^{\prime}\right)$, the results from Part 1 yields (use (i) of Proposition 4.10 to bound $\left.\theta^{\prime}(s)\right)$

$$
\begin{aligned}
& \left\|\frac{q_{-}(s)}{1+|y|^{M+1}}\right\|_{L^{\infty}} \leq e^{-\frac{M+1}{2}(s-\tau)}\left\|\frac{q_{-}(\tau)}{1+|y|^{M+1}}\right\|_{L^{\infty}} \\
& +\int_{\tau}^{s} e^{-\frac{M+1}{2}\left(s-s^{\prime}\right)}\left(\sqrt{1+\delta^{2}}+\left\|\left|V_{1}\right|+\left|V_{2}\right|\right\|_{L^{\infty}}\right)\left\|\frac{q_{-}}{1+|y|^{M+1}}\right\|_{L^{\infty}} d s^{\prime} \\
& +C(M) \int_{\tau}^{s} e^{-\frac{M+1}{2}\left(s-s^{\prime}\right)}\left[\frac{A^{(M+1)^{2}+5}}{\left(s^{\prime}\right)^{\frac{M+3}{4}}}+\frac{A^{(M+2) \bar{p}}}{\left(s^{\prime}\right)^{\frac{\bar{p}-1}{2}}} \frac{1}{\left(s^{\prime}\right)^{\frac{M+2}{2}}}+\frac{A^{M}}{\left(s^{\prime}\right)^{\frac{M+2}{2}}}\right] d s^{\prime} .
\end{aligned}
$$


Since we have already fixed $M$ in (50) such that

$$
M \geq 4\left(\sqrt{1+\delta^{2}}+1+2 \max _{i=1,2, y \in \mathbb{R}, s \geq 1}\left|V_{i}(y, s)\right|\right),
$$

using Gronwall's lemma or Maximum principle and (70), we deduce that

$$
\begin{aligned}
e^{\frac{M+1}{2} s}\left\|\frac{q_{-}(s)}{1+|y|^{M+1}}\right\|_{L^{\infty}} & \leq e^{\frac{M+1}{4}(s-\tau)} e^{\frac{M+1}{2} \tau}\left\|\frac{q_{-}(\tau)}{1+|y|^{M+1}}\right\|_{L^{\infty}} \\
& +e^{\frac{M+1}{2} s} 2^{\frac{M+3}{4}}\left[\frac{A^{(M+1)^{2}+5}}{s^{\frac{M+3}{4}}}+\frac{A^{(M+2) \bar{p}}}{s^{\frac{\bar{p}-1}{2}}} \frac{1}{\left(s^{\prime}\right)^{\frac{M+2}{2}}}+\frac{A^{M}}{s^{\frac{M+2}{2}}}\right]
\end{aligned}
$$

which concludes the proof of the last but one identity in (iv) of Proposition 4.10,

\subsubsection{The outer region: $q_{e}$}

Here, we finish the proof of Proposition 4.10 by proving the last inequality in (iv). Since $q(s) \in \mathcal{V}_{A}(s)$ for all $s \in\left[\tau, s_{1}\right]$, it holds from Claim 4.8 and Proposition 4.10 that

$$
\|q(s)\|_{L^{\infty}\left(|y|<2 K s^{1 / 4}\right)} \leq C \frac{A^{M+1}}{s^{1 / 4}} \text { and }\left|\theta^{\prime}(s)\right| \leq \frac{C A^{5}}{s^{3 / 2}} .
$$

Then, we derive from (38) an equation satisfied by $q_{e}$, where $q_{e}$ is defined by (41):

$$
\begin{aligned}
\frac{\partial q_{e}}{\partial s} & =\mathcal{L}_{0} q_{e}-\frac{1}{p-1} q_{e}+(1-\chi) e^{\frac{i \delta}{p-1} s}\left\{L\left(q, \theta^{\prime}, y, s\right)+R^{*}\left(\theta^{\prime}, y, s\right)\right\} \\
& -e^{\frac{i \delta}{p-1} s} q(s)\left(\partial_{s} \chi+\Delta \chi+\frac{1}{2} y \cdot \nabla \chi\right)+2 e^{\frac{i \delta}{p-1} s} \operatorname{div}(q(s) \nabla \chi) .
\end{aligned}
$$

Writing this equation in its integral form and using the maximum principle satisfied by $e^{\tau \mathcal{L}_{0}}$ (see Lemma A.1, see Apendix below), we write

$$
\begin{aligned}
\left\|q_{e}(s)\right\|_{L^{\infty}} & \leq e^{-\frac{s-\tau}{p-1}}\left\|q_{e}(\tau)\right\|_{L^{\infty}}, \\
& +\int_{\tau}^{s} e^{-\frac{s-s^{\prime}}{p-1}}\left(\left\|(1-\chi) L\left(q, \theta^{\prime}, y, s^{\prime}\right)\right\|_{L^{\infty}}+\left\|(1-\chi) R^{*}\left(\theta^{\prime}, y, s^{\prime}\right)\right\|_{L^{\infty}}\right) d s^{\prime} \\
& +\int_{\tau}^{s} e^{-\frac{s-s^{\prime}}{p-1}}\left\|q\left(s^{\prime}\right)\left(\partial_{s} \chi+\Delta \chi+\frac{1}{2} y \cdot \nabla \chi\right)\right\|_{L^{\infty}} d s^{\prime} \\
& +\int_{\tau}^{s} e^{-\frac{s-s^{\prime}}{p-1}} \frac{1}{\sqrt{1-e^{-\left(s-s^{\prime}\right)}}}\left\|q\left(s^{\prime}\right) \nabla \chi\right\|_{L^{\infty}} d s^{\prime} .
\end{aligned}
$$

Let us bounds the norms in the three last lines of this inequality.

First from (40) and (116)

$$
\begin{aligned}
&\left\|q\left(s^{\prime}\right)\left(\partial_{s} \chi+\Delta \chi+\frac{1}{2} y \cdot \nabla \chi\right)\right\|_{L^{\infty}} \leq C\left(1+\frac{1}{K^{2} s^{\prime}}\right)\left\|q\left(s^{\prime}\right)\right\|_{L^{\infty}\left(|y|<2 K s^{\prime 1 / 4}\right)} \\
& \leq C \frac{A^{M+1}}{\left(s^{\prime}\right)^{1 / 4}}, \\
&\left\|q\left(s^{\prime}\right) \nabla \chi\right\|_{L^{\infty}} \leq \frac{C}{K\left(s^{\prime}\right)^{1 / 4}}\left\|q\left(s^{\prime}\right)\right\|_{L^{\infty}\left(|y|<2 K\left(s^{\prime}\right)^{1 / 4}\right)} \leq C \frac{A^{M+1}}{\sqrt{s^{\prime}}},
\end{aligned}
$$


for $s^{\prime}$ large enough.

Second note that the residual term $(1-\chi) R^{*}$ is small as well. Indeed, recalling the bound (45) on $R$, we write from the definition of $R^{*}$ (39) and (116):

$$
\left\|(1-\chi) R^{*}\left(\theta^{\prime}, y, s^{\prime}\right)\right\|_{L^{\infty}} \leq \frac{C}{\left(s^{\prime}\right)^{1 / 4}}+\left|\theta^{\prime}\left(s^{\prime}\right)\right| \leq \frac{C}{\left(s^{\prime}\right)^{1 / 4}}
$$

for $s^{\prime}$ large enough.

Third, the term $(1-\chi) L\left(q, \theta^{\prime}, y, s^{\prime}\right)$ given in (39) is less than $\epsilon\left|q_{e}\right|$ with $\epsilon=\frac{1}{2(p-1)}$. Indeed, it holds from (116) that:

$$
\begin{aligned}
& \left\|(1-\chi) L\left(q, \theta^{\prime}, y, s^{\prime}\right)\right\|_{L^{\infty}} \\
& \leq C\left\|q_{e}\left(s^{\prime}\right)\right\|_{L^{\infty}}\left(\left\|\varphi\left(s^{\prime}\right)\right\|_{L^{\infty}\left(|y| \geq K s^{\prime 1 / 4}\right)}^{p-1}+\left\|q\left(s^{\prime}\right)\right\|_{L^{\infty}\left(|y| \geq K s^{\prime 1 / 4}\right)}^{p-1}+\frac{1}{s^{\prime}}+\left|\theta^{\prime}(s)\right|\right), \\
& \leq \frac{1}{2(p-1)}\left\|q_{e}\left(s^{\prime}\right)\right\|_{L^{\infty}}
\end{aligned}
$$

whenever $K$ and $s^{\prime}$ are large (in order to ensure that $\left\|\varphi\left(s^{\prime}\right)\right\|_{L^{\infty}\left(|y|>K s^{\prime 1 / 4}\right)}$ is small).

Notice that it is only here that we need the fact that $K$ is big enough. Using estimates (116), (118), (119), (120) and (121), we write

$$
\begin{aligned}
\left\|q_{e}(s)\right\|_{L^{\infty}} & \leq e^{-\frac{s-\tau}{p-1}}\left\|q_{e}(\tau)\right\|_{L^{\infty}} \\
& +\int_{\tau}^{s} e^{-\frac{s-s^{\prime}}{p-1}}\left(\frac{1}{2(p-1)}\left\|q_{e}\left(s^{\prime}\right)\right\|_{L^{\infty}}+C \frac{A^{M+1}}{\left(s^{\prime}\right)^{\frac{1}{4}}}+C \frac{A^{M+1}}{\left(s^{\prime}\right)^{\frac{1}{2}}} \frac{1}{\sqrt{1-e^{-\left(s-s^{\prime}\right)}}}\right) d s^{\prime} .
\end{aligned}
$$

Using Gronwall's inequality or Maximum principle, we end-up with

$$
\left\|q_{e}(s)\right\|_{L^{\infty}} \leq e^{-\frac{(s-\tau)}{2(p-1)}}\left\|q_{e}(\tau)\right\|_{L^{\infty}}+\frac{C A^{M+1}}{\tau^{\frac{1}{4}}}(s-\tau+\sqrt{s-\tau})
$$

which concludes the proof of Proposition 4.10.

\section{Single point blow-up and final profile}

In this section, we prove Theorem 1. Here, we use the solution of problem (42)-(58) constructed in the last section to exhibit a blow-up solution of equation (11) and prove Theorem 1 ,

(i) Consider $(q(s), \theta(s))$ constructed in Section 4 such that (54) holds. From (54) and the properties of the shrinking set given in Claim 4.8, we see that $\theta(s) \rightarrow \theta_{0}$ as $s \rightarrow \infty$ such that

$$
\left|\theta(s)-\theta_{0}\right| \leq C A^{5} \int_{s}^{\infty} \frac{1}{\tau^{\frac{3}{2}}} d \tau \leq \frac{C A^{5}}{\sqrt{s}} \text { and }\|q(s)\|_{L^{\infty}(\mathbb{R})} \leq \frac{C_{0}(K, A)}{\sqrt{s}} .
$$

Introducing $w(y, s)=e^{i(\mu \log s+\theta(s))}(\varphi(y, s)+q(y, s))$, we see that $w$ is a solution of equation (14) that satisfies for all $s \geq \log T$ and $y \in \mathbb{R}$,

$$
\left|w(y, s)-e^{i \theta_{0}+i \mu \log s} \varphi(y, s)\right| \leq C\|q(s)\|_{L^{\infty}}+C\left|\theta(s)-\theta_{0}\right| \leq \frac{C_{0}}{s^{\frac{1}{4}}} .
$$


Introducing

$$
u(x, t)=e^{-i \theta_{0}} \kappa^{i \delta}(T-t)^{\frac{1+i \delta}{p-1}} w\left(\frac{y}{\sqrt{T-t}},-\log (T-t)\right),
$$

we see from (13) and the definition of $\varphi$ (34) that $u$ is a solution of equation (11) defined for all $(x, T) \in \mathbb{R} \times[0, T)$ which satisfies (9).

If $x_{0}=0$. It remains to prove that when $x_{0} \neq 0, x_{0}$ is not a blow-up point. The following result from Giga and Kohn GK89 allows us to conclude:

Proposition 5.1 (Giga and Kohn - No blow-up under the ODE threshold) For all $C_{0}>0$, there is $\eta_{0}>0$ such that if $v(\xi, \tau)$ solves

$$
\left|v_{t}-\Delta v\right| \leq C_{0}\left(1+|v|^{p}\right)
$$

and satisfies

$$
|v(\xi, \tau)| \leq \eta_{0}(T-t)^{-1 /(p-1)}
$$

for all $(\xi, \tau) \in B(a, r) \times\left[T-r^{2}, T\right)$ for some $a \in \mathbb{R}$ and $r>0$, then $v$ does not blow up at $(a, T)$.

Proof: See Theorem 2.1 page 850 in [GK89.

Indeed, we see from (9) and (34) that

$$
\sup _{\left|x-x_{0}\right| \leq \frac{\left|x_{0}\right|}{2}}(T-t)^{\frac{1}{p-1}}|u(x, t)| \leq\left|\varphi_{0}\left(\frac{\left|x_{0}\right| / 2}{\sqrt{(T-t)}|\log (T-t)|}\right)\right|+\frac{C}{|\log (T-t)|^{\frac{1}{4}}} \rightarrow 0
$$

as $t \rightarrow T, x_{0}$ is not a blow-up point of $u$ from Proposition 5.1. This concludes the proof of (i) of Theorem 1 .

(ii) Arguing as Merle did in [Mer92, we derive the existence of a blow-up profile $u^{*} \in$ $C^{2}\left(\mathbb{R}^{*}\right)$ such that $u(x, t) \rightarrow u^{*}(x)$ as $t \rightarrow T$, uniformly on compact sets of $\mathbb{R}^{*}$. The profile $u^{*}(x)$ is not defined at the origin. In the following, we would like to find its equivalent as $x \rightarrow 0$ and show that it is in fact singular at the origin. We argue as in Masmoudi and Zaag [MZ08]. Consider $K_{0}>0$ to be fixed large enough later. If $x_{0} \neq 0$ is small enough, we introduce for all $(\xi, \tau) \in \mathbb{R} \times\left[-\frac{t_{0}\left(x_{0}\right)}{T-t_{0}\left(x_{0}\right)}, 1\right)$,

$$
\begin{aligned}
v\left(x_{0}, \xi, \tau\right) & =\left(T-t_{0}\left(x_{0}\right)\right)^{\frac{1+i \delta}{p-1}} v(x, t), \\
\text { where, } x & =x_{0}+\xi \sqrt{T-t_{0}\left(x_{0}\right)}, t=t_{0}\left(x_{0}\right)+\tau\left(T-t_{0}\left(x_{0}\right)\right),
\end{aligned}
$$

and $t_{0}\left(x_{0}\right)$ is uniquely determined by

$$
\left|x_{0}\right|=K_{0} \sqrt{\left(T-t_{0}\left(x_{0}\right)\right)\left|\log \left(T-t_{0}\left(x_{0}\right)\right)\right|^{\frac{1}{2}}} .
$$

From the invariance of problem (11) under dilation, $v\left(x_{0}, \xi, \tau\right)$ is also a solution of (1) on its domain. From (124), (125), (34), we have

$$
\sup _{|\xi|<2\left|\log \left(T-t_{0}\left(x_{0}\right)\right)\right|^{1 / 8}}\left|v\left(x_{0}, \xi, 0\right)-\varphi_{0}\left(K_{0}\right)\right| \leq \frac{C}{\left|\log \left(T-t_{0}\left(x_{0}\right)\right)\right|^{\frac{1}{8}}} \rightarrow 0 \text { as } x_{0} \rightarrow 0 .
$$


Using the continuity with respect to initial data for problem (11) associated to a spacelocalization in the ball $B\left(0,|\xi|<\left|\log \left(T-t_{0}\left(x_{0}\right)\right)\right|^{1 / 8}\right)$, we show as in Section 4 of [Zaa98] that

$$
\sup _{|\xi| \leq\left|\log \left(T-t_{0}\left(x_{0}\right)\right)\right|^{1 / 8}, 0 \leq \tau<1}\left|v\left(x_{0}, \xi, \tau\right)-U_{K_{0}}(\tau)\right| \leq \epsilon\left(x_{0}\right) \text { as } x_{0} \rightarrow 0,
$$

where $U_{K_{0}}(\tau)=\left((p-1)(1-\tau)+b K_{0}^{2}\right)^{-\frac{1+i \delta}{p-1}}$ is the solution of the PDE (11) with constant initial data $\varphi_{0}\left(K_{0}\right)$. Making $\tau \rightarrow 1$ and using (124), we see that

$$
\begin{aligned}
u^{*}\left(x_{0}\right)=\lim _{t \rightarrow T} v(x, t) & =\left(T-t_{0}\left(x_{0}\right)\right)^{-\frac{1+i \delta}{p-1}}\left|\log \left(T-t_{0}\left(x_{0}\right)\right)\right|^{i \mu} \lim _{\tau \rightarrow 1} v\left(x_{0}, 0, \tau\right) \\
& \sim\left(T-t_{0}\left(x_{0}\right)\right)^{-\frac{1+i \delta}{p-1}} \mid \log \left(T-t_{0}\left(x_{0}\right)\right)^{i \mu} U_{K_{0}}(1)
\end{aligned}
$$

as $x_{0} \rightarrow 0$. Since we have from (125)

$$
\log \left(T-t_{0}\left(x_{0}\right)\right) \sim 2 \log \left|x_{0}\right| \text { and } T-t_{0}\left(x_{0}\right) \sim \frac{\left|x_{0}\right|^{2}}{\sqrt{2} K_{0}^{2} \sqrt{|\log | x_{0} \mid}},
$$

as $x_{0} \rightarrow 0$, this yields (ii) of Theorem 1 and concludes the proof of Theorem 1 ,

\section{A Spectral properties of $\mathcal{L}_{0}$}

In this Appendix, we give some properties associated to the operator $\mathcal{L}_{0}$, defined in (39);

$$
\mathcal{L}_{0} v=\Delta v-\frac{1}{2} y \cdot \nabla v
$$

Indeed as stated by [BK94], we have the following Mehler's kernel for the semigroup $\mathcal{L}_{0}$ l:

$$
e^{s \mathcal{L}_{0}}(y, x)=\frac{1}{\left[4 \pi\left(1-e^{-s}\right)\right]^{N / 2}} \exp \left[-\frac{\left|x-y e^{-\frac{s}{2}}\right|}{4\left(1-e^{-s}\right)}\right] .
$$

In the following, we give some properties associated to the kernel.

Lemma A.1 a) The semigroup associated to $\mathcal{L}_{0}$ satisfies the maximum principle:

$$
\left\|e^{s \mathcal{L}_{0}} \varphi\right\|_{L^{\infty}} \leq\|\varphi\|_{L^{\infty}}
$$

b) Moreover, we have

$$
\left\|e^{s \mathcal{L}_{0}} \operatorname{div}(\varphi)\right\|_{L^{\infty}} \leq \frac{C}{\sqrt{1-e^{-s}}}\|\varphi\|_{L^{\infty}}
$$

where $C$ is a constant.

Proof: a) It follows directly by part, this also follows from the definition of the semigroup (126).

b) Using an integration by part, this also follows from the definition of the semigroup (126). 
Lemma A.2 There exists a constant $C$ such that if $\phi$ satisfies

$$
\forall x \in \mathbb{R}|\phi(x)| \leq\left(1+|x|^{M+1}\right)
$$

then for all $y \in \mathbb{R}$, we have

$$
\left|e^{s \mathcal{L}_{0}} P_{-}(\phi(y))\right| \leq C e^{-\frac{M+1}{2} s}\left(1+|y|^{M+1}\right)
$$

Proof: This also follows directly from the semigroup's definition, through an integration by part, for a similar case see page 556-558 from [BK94.

Moreover, we have the following useful lemma about $P_{-}$.

Lemma A.3 For all $k \geq 0$, we have

$$
\left\|\frac{P_{-}(\phi)}{1+|y|^{M+k}}\right\|_{L^{\infty}} \leq C\left\|\frac{\phi}{1+|y|^{M+k}}\right\|
$$

Proof: Using (49), we have

$$
\left|\phi_{n}\right| \leq C\left\|\frac{\phi}{1+|y|^{M+k}}\right\|_{L^{\infty}} .
$$

Since for all $m \leq M,\left|h_{m}(y)\right| \leq C\left(1+|y|^{m+k}\right)$ and

$$
|\phi| \leq C\left\|\frac{\phi}{1+|y|^{M+k}}\right\|_{L^{\infty}}\left(1+|y|^{m+k}\right),
$$

the result follows from definition (48) of $\phi$.

\section{B Details of expansions of the fourth term of equation (39) $V_{1} q+V_{2} \bar{q}$}

In the following, we will try to expand each term of $V_{1}(y, s)$ and $V_{2}(y, s)$ as a power series of $\frac{1}{s}$ as $s \rightarrow \infty$, uniformly for $|y| \leq C s^{\frac{1}{4}}$, with $C$ a positive constant.

$$
\begin{aligned}
& \left|\varphi_{0}(z)+\frac{a}{s^{1 / 2}}(1+i \delta)\right|^{p-1} \\
& =\kappa^{p-1}\left(1+\frac{(p-1) a}{\kappa s^{1 / 2}}+\frac{a^{2}(p-1)^{2}}{\kappa^{2} s}-\frac{b}{(p-1)} \frac{y^{2}}{s^{1 / 2}}+\frac{a^{3}}{3 \kappa^{3} s^{3 / 2}}(p-1)(p-3)(2 p-1)\right. \\
& -\frac{2 a b}{\kappa} \frac{y^{2}}{s}+\frac{a^{2} b(p-3)(1-2 p)}{\kappa^{2}(p-1)} \frac{y^{2}}{s^{3 / 2}} \\
& +\frac{b^{2}}{(p-1)^{2}} \frac{y^{4}}{s}+\frac{a b^{2}}{2 \kappa(p-1)^{3}}\left[(p+1)(p-2)+2(p-1)(p-3)+2(p-1)^{2}\right] \frac{y^{4}}{s^{3 / 2}} \\
& \left.-\frac{b^{3}}{(p-1)^{3}} \frac{y^{6}}{s^{3 / 2}}+O\left(\frac{1}{s^{2}}\right)+O\left(\frac{y^{8}}{s^{2}}\right)\right) . \\
& V_{1}=(1+i \delta) \frac{p+1}{2}\left(\left|\varphi_{0}(z)+\frac{a}{s^{1 / 2}}(1+i \delta)\right|^{p-1}-\frac{1}{p-1}\right) \\
& =\left(\frac{1}{s^{1 / 2}} W_{1,1}+\frac{1}{s} W_{1,2}+O\left(\frac{1}{s^{3 / 2}}\right)+O\left(\frac{y^{6}}{s^{3 / 2}}\right)\right)
\end{aligned}
$$


Recalling that $b=\frac{(p-1)^{2}}{8 \sqrt{p(p+1)}}, \quad a=\frac{\kappa}{4 \sqrt{p(p+1)}}$, , we have

$$
\begin{array}{ll}
W_{1,1} & =-(1+i \delta) \frac{b(p+1)}{2(p-1)^{2}} h_{2}(y) \\
& F_{2}\left(W_{1,1} \tilde{q}_{2} \tilde{h}_{2}\right)=-32(1+i \delta)^{2} \frac{b(p+1)}{(p-1)^{2}} \tilde{q}_{2} \\
& \tilde{P}_{2}\left(W_{1,1} \tilde{q}_{2} \tilde{h}_{2}\right)=32 \frac{b(p+1)}{(p-1)} \tilde{q}_{2}, \\
& F_{0}\left(W_{1,1} \tilde{q}_{2} \tilde{h}_{2}\right)=-4(1-p+2 i \delta) \frac{b(p+1)}{(p-1)^{2}} \tilde{q}_{2} \\
& \hat{P}_{0}\left(W_{1,1} \tilde{q}_{2} \tilde{h}_{2}\right)=-4 \delta \frac{b(p+1)^{2}}{(p-1)^{2}} \tilde{q}_{2} \\
& =(1+i \delta) \frac{b^{2}(p+1)}{2(p-1)^{3}}\left(y^{4}-4 y^{2}+4\right)=(1+i \delta) \frac{b^{2}(p+1)}{2(p-1)^{3}} h_{2}^{2}(y), \\
W_{1,2} & =60(1+i \delta)^{2} \frac{b^{2}(p+1)}{(p-1)^{3}} \tilde{q}_{2} \\
F_{2}\left(W_{1,2} \tilde{q}_{2} \tilde{h}_{2}\right) & =-60 \frac{b^{2}(p+1)}{(p-1)^{2}} \tilde{q}_{2} \\
\tilde{P}_{2}\left(W_{1,2} \tilde{q}_{2} \tilde{h}_{2}\right) &
\end{array}
$$

Let us now expand the term $V_{2}$;

$$
\begin{aligned}
& \left|\varphi_{0}(z)+\frac{a}{s^{1 / 2}}(1+i \delta)\right|^{p-3} \\
& =\kappa^{p-3}\left(1+\frac{2 a}{\kappa s^{1 / 2}}+\frac{a^{2}(p+1)}{\kappa^{2} s}\right)^{\frac{p-3}{2}}\left(1-2 \frac{b}{(p-1)^{2}} \frac{y^{2}}{s^{1 / 2}}-2 \frac{a b}{\kappa(p-1)} \frac{y^{2}}{s}+\frac{b^{2}(p+1)}{(p-1)^{4}} \frac{y^{4}}{s}\right. \\
& \left.+2 \frac{a^{2} b(3 p-1)}{\kappa^{2}(p-1)^{2}} \frac{y^{2}}{s^{3 / 2}}+\frac{a b^{2}(p+1)(p-2)}{\kappa(p-1)^{4}} \frac{y^{4}}{s^{3 / 2}}-\frac{2}{3} \frac{b^{3} p(p+1)}{(p-1)^{6}} \frac{y^{6}}{s^{3 / 2}}+O\left(\frac{y^{6}}{s^{2}}\right)+O\left(\frac{y^{8}}{s^{2}}\right)\right)^{\frac{p-3}{2}} \\
& =\kappa^{p-3}\left(1+\frac{a(p-3)}{\kappa} \frac{1}{s^{1 / 2}}+\frac{a^{2}(p-3)(p-2)}{\kappa^{2}} \frac{1}{s}+\frac{2}{3} \frac{a^{3}(p-1)(p-3)(p-5)}{\kappa^{3}} \frac{1}{s^{3 / 2}}+O\left(\frac{1}{s^{2}}\right)\right) \\
& *\left(1-\frac{b(p-3)}{(p-1)^{2}} \frac{y^{2}}{s^{1 / 2}}-\frac{a b(p-3)}{\kappa(p-1)} \frac{y^{2}}{s}+\frac{b^{2}(p-3)(p-2)}{(p-1)^{4}} \frac{y^{4}}{s}+\frac{a b^{2}(p-3)^{2}(2 p-1)}{2 \kappa(p-1)^{4}} \frac{y^{4}}{s^{3 / 2}}-\frac{b^{3}(p-2)(p-3)(3 p-5)}{3(p-1)^{6}} \frac{y^{6}}{s^{3 / 2}}+O\left(\frac{y^{6}}{s^{2}}\right)+O\left(\frac{y^{8}}{s^{2}}\right)\right) \\
& =\kappa^{p-3}\left(1+\frac{a(p-3)}{\kappa} \frac{1}{s^{1 / 2}}+\frac{a^{2}(p-3)(p-2)}{\kappa^{2}} \frac{1}{s}+\frac{2}{3} \frac{a^{3}(p-1)(p-3)(p-5)}{\kappa^{3}} \frac{1}{s^{3 / 2}}-\frac{b(p-3)}{(p-1)^{2}} \frac{y^{2}}{s^{1 / 2}}-\frac{2 a b(p-3)(p-2)}{\kappa(p-1)^{2}} \frac{y^{2}}{s}\right. \\
& \left.+\frac{b^{2}(p-3)(p-2)}{(p-1)^{4}} \frac{y^{4}}{s}+\frac{a b^{2}(p-3)^{2}(4 p-5)}{2 \kappa(p-1)^{4}} \frac{y^{4}}{s^{3 / 2}}-\frac{b^{3}(p-2)(p-3)(3 p-5)}{3(p-1)^{6}} \frac{y^{6}}{s^{3 / 2}}+O\left(\frac{1}{s^{2}}\right)+O\left(\frac{y^{8}}{s^{2}}\right)\right) \\
& \left(\varphi_{0}(z)+\frac{a}{s^{1 / 2}}(1+i \delta)\right)^{2} \\
& =\kappa^{2}\left(1+\frac{a}{\kappa \sqrt{s}}(1+i \delta)-\frac{b}{(p-1)^{2}}(1+i \delta) \frac{y^{2}}{s^{1 / 2}}+i \frac{b^{2} \delta(p+1)}{2(p-1)^{4}} \frac{y^{4}}{s}-\frac{b^{3} \delta(p+1)}{6(p-1)^{6}}(\delta+i(1-2 p)) \frac{y^{6}}{s^{3 / 2}}+O\left(\frac{y^{8}}{s^{2}}\right)\right)^{2} \\
& =\kappa^{2}\left(1+\frac{2 a}{\kappa \sqrt{s}}(1+i \delta)-\frac{2 b}{(p-1)^{2}}(1+i \delta) \frac{y^{2}}{s^{1 / 2}}+i \frac{b^{2} \delta(p+1)}{(p-1)^{4}} \frac{y^{4}}{s}+\frac{a^{2}}{\kappa^{2}}(1+i \delta)^{2} \frac{1}{s}-\frac{2 a b}{\kappa(p-1)^{2}}(1+i \delta)^{2} \frac{y^{2}}{s}\right. \\
& \left.+\frac{b^{2}(1+i \delta)^{2}}{(p-1)^{4}} \frac{y^{4}}{s}+\frac{a b^{2} \delta(p+1)(-\delta+i)}{\kappa(p-1)^{4}} \frac{y^{4}}{s^{3 / 2}}+\frac{(\delta-i) b^{3} \delta(p+1)}{(p-1)^{6}} \frac{y^{6}}{s^{3 / 2}}-\frac{b^{3} \delta(p+1)}{3(p-1)^{6}}(\delta+i(1-2 p)) \frac{y^{6}}{s^{3 / 2}}+O\left(\frac{y^{8}}{s^{2}}\right)\right) \\
& =\kappa^{2}\left(1+(1+i \delta) \frac{2 a}{\kappa} \frac{1}{\sqrt{s}}+((1-p)+i 2 \delta) \frac{a^{2}}{\kappa^{2}} \frac{1}{s}-(1+i \delta) \frac{2 b}{(p-1)^{2}} \frac{y^{2}}{s^{1 / 2}}-((1-p)+i 2 \delta) \frac{2 a b}{\kappa(p-1)^{2}} \frac{y^{2}}{s}\right. \\
& \left.+(1-p+i \delta(p+3)) \frac{b^{2}}{(p-1)^{4}} \frac{y^{4}}{s}+\frac{a b^{2}(p+1)(-p+i \delta)}{\kappa(p-1)^{4}} \frac{y^{4}}{s^{3 / 2}}+\frac{2}{3} \frac{b^{3}(p+1)}{(p-1)^{6}}(p+i \delta(p-2)) \frac{y^{6}}{s^{3 / 2}}+O\left(\frac{y^{6}}{s^{4}}\right)+O\left(\frac{y^{8}}{s^{4}}\right)\right) \\
& W_{2,1}=-(1+i \delta) \frac{p-1}{2} \frac{b}{(p-1)^{3}}(p-1+2 i \delta) h_{2}(y) \\
& F_{2}\left(W_{2,1} \tilde{q}_{2} \overline{\tilde{h}_{2}}\right)=-32\left((p-1+2 i \delta)(p+1) \frac{b}{(p-1)^{2}} \tilde{q}_{2}\right. \\
& \tilde{P}_{2}\left(W_{2,1} \tilde{q}_{2} \overline{\tilde{h}_{2}}\right)=-32 \frac{b(p+1)}{p-1} \tilde{q}_{2} \\
& F_{0}\left(W_{2,1} \tilde{q}_{2} \overline{\tilde{h}_{2}}\right)=-4\left((p-1+2 i \delta)(p+1) \frac{b}{(p-1)^{2}} \tilde{q}_{2}\right. \\
& \hat{P}_{0}\left(W_{2,1} \tilde{q}_{2} \overline{\tilde{h}_{2}}\right)=4 \delta \frac{b(p+1)(p-3)}{(p-1)^{2}} \tilde{q}_{2} \text {, }
\end{aligned}
$$




$$
\begin{aligned}
W_{2,2}= & \frac{p-1}{2}(1+i \delta)\left\{\frac{a^{2}}{\kappa^{2} s}\left(p^{2}-4 p+1+i 2 \delta(p-2)\right)\right. \\
& -y^{2} \frac{2 a b}{\kappa(p-1)^{2}}\left(p^{2}-4 p+1+i 2 \delta(p-2)\right) \\
& \left.+y^{4} \frac{b^{2}}{(p-1)^{4}}\left(p^{2}-4 p+1+i 3 \delta(p-1)\right)\right\} \\
= & (1+i \delta) \frac{b^{2}}{2(p-1)^{3}}\left\{4\left(p^{2}-4 p+1+i 2 \delta(p-2)\right)\right. \\
& -4 y^{2}\left(p^{2}-4 p+1+i 2 \delta(p-2)\right) \\
& \left.+y^{4}\left(p^{2}-4 p+1+i 3 \delta(p-1)\right)\right\} . \\
= & (1+i \delta) \frac{b^{2}}{2(p-1)^{3}}\left[\left(p^{2}-4 p+1\right) h_{2}^{2}(y)\right. \\
& \left.+i \delta\left(8(p-2)\left(1-y^{2}\right)+y^{4} 3(p-1)\right)\right],
\end{aligned}
$$

Then, we can write

$$
W_{2,2} \tilde{q}_{2} \overline{\tilde{h}_{2}}=(p+1) \frac{b^{2}}{2(p-1)^{3}}\left[\left(p^{2}-4 p+1\right) h_{2}^{3}(y)+i \delta\left(8(p-2)\left(1-y^{2}\right)+y^{4} 3(p-1)\right) h_{2}(y)\right]
$$

and we obtain

$$
\tilde{P}_{2}\left(W_{2,2} \tilde{q}_{2} \overline{\tilde{h}_{2}}\right)=60(p+1) \frac{b^{2}}{2(p-1)^{3}}\left(p^{2}-4 p+1\right) \tilde{q}_{2}
$$

\section{Details of expansions of the sixth term of equation (39) $R^{*}\left(\theta^{\prime}, y, s\right)$}

Using the definition of $\varphi$, the fact that $\varphi_{0}$ satisfies (37) and (42), we see that $R^{*}$ is in fact a function of $\theta^{\prime}, z=\frac{y}{s^{1 / 4}}$ and $s$ that can be written as

$$
\begin{aligned}
& R^{*}\left(\theta^{\prime}, y, s\right)=\frac{1}{4} \frac{z}{s} \nabla_{z} \varphi_{0}(z)+\frac{1}{2} \frac{a}{s^{3 / 2}}(1+i \delta)+\frac{1}{s^{1 / 2}} \Delta_{z} \varphi_{0}(z) \\
& -\frac{a(1+i \delta)^{2}}{(p-1) s^{1 / 2}}+\left(F\left(\varphi_{0}(z)+\frac{a}{s^{1 / 2}}(1+i \delta)\right)-F\left(\varphi_{0}\right)\right) \\
& -i \frac{\mu}{s}\left(\varphi_{0}(z)+\frac{a}{s^{1 / 2}}(1+i \delta)\right)-i \theta^{\prime}(s)\left(\varphi_{0}(z)+\frac{a}{s^{1 / 2}}(1+i \delta)\right) \text { with, } F(u)=(1+i \delta)|u|^{p-1} u .
\end{aligned}
$$

In the following, we will try to expand each term of (129) as a power series of $\frac{1}{s}$ as $s \rightarrow \infty$, uniformly for $|y| \leq C s^{\frac{1}{4}}$, with $C$ a positive constant.

- $\frac{1}{4} \frac{z}{s} \nabla_{z} \varphi_{0}(z)$ :

$$
\begin{aligned}
\frac{1}{4} \frac{z}{s} \nabla_{z} \varphi_{0}(z) & =-\frac{1}{2} \kappa \frac{1+i \delta}{p-1} \frac{b}{p-1} \frac{z^{2}}{s}\left(1+\frac{b}{p-1} z^{2}\right)^{-\frac{p+i \delta}{p-1}} \\
& =-\frac{1}{2} \kappa \frac{1+i \delta}{p-1} \frac{b}{p-1} \frac{y^{2}}{s^{3 / 2}}\left\{1-\frac{p+i \delta}{p-1} \frac{b}{p-1} \frac{y^{2}}{s^{1 / 2}}+O\left(\frac{y^{4}}{s}\right)\right\} \\
& =-\frac{1}{2} \kappa(1+i \delta) \frac{b}{(p-1)^{2}} \frac{y^{2}}{s^{3 / 2}}+O\left(\frac{y^{4}}{s^{2}}\right)+O\left(\frac{y^{6}}{s^{2}}\right) .
\end{aligned}
$$

- $\frac{1}{s^{1 / 2}} \Delta_{z} \varphi_{0}(z)$ : 


$$
\begin{aligned}
& \frac{1}{s^{1 / 2}} \Delta_{z} \varphi_{0}(z)= \\
& -2 \kappa(1+i \delta) \frac{b}{(p-1)^{2}} \frac{1}{s^{1 / 2}}\left(1+\frac{b}{p-1} z^{2}\right)^{-\frac{p+i \delta}{p-1}}+4 \kappa \frac{1+i \delta}{p-1} \frac{p+i \delta}{p-1} \frac{b^{2}}{(p-1)^{2}} \frac{z^{2}}{s^{1 / 2}}\left(1+\frac{b}{p-1} z^{2}\right)^{-\frac{2 p-1+i \delta}{p-1}} \\
& =2 \kappa(1+i \delta) \frac{b}{(p-1)^{2}} \frac{1}{s^{1 / 2}}\left\{-1+\frac{b(p+i \delta)}{(p-1)^{2}} \frac{y^{2}}{s^{1 / 2}}-\frac{b^{2}(p+i \delta)(2 p-1+i \delta)}{2(p-1)^{4}} \frac{y^{4}}{s}\right. \\
& \left.+2 \frac{p+i \delta}{p-1} \frac{b}{p-1} \frac{y^{2}}{s^{1 / 2}}\left(1-\frac{b(2 p-1+i \delta)}{(p-1)^{2}} \frac{y^{2}}{s^{1 / 2}}\right)+O\left(\frac{y^{6}}{s^{3 / 2}}\right)\right\} \\
& =-2 \kappa(1+i \delta) \frac{b}{(p-1)^{2}} \frac{1}{s^{1 / 2}}+i 6 \kappa \frac{b^{2} \delta(p+1)}{(p-1)^{2}} \frac{y^{2}}{s}+5 \kappa \frac{b^{3} \delta(p+1)}{(p-1)^{6}}(\delta-i(2 p-1)) \frac{y^{4}}{s^{3 / 2}}+O\left(\frac{y^{6}}{s^{2}}\right) .
\end{aligned}
$$

- $F\left(\varphi_{0}(z)+\frac{a}{s^{1 / 2}}(1+i \delta)\right)$ We start by the following term and recall that we work in the critical case $\delta^{2}=p$ and we expand this term as a power series of $\frac{1}{s}$ as $s \rightarrow \infty$, uniformly for $|y| \leq C s^{\frac{1}{4}}$, with $C$ a positive constant. 


$$
\begin{aligned}
& \left|\varphi_{0}(z)+\frac{a}{s^{1 / 2}}(1+i \delta)\right|^{p-1} \\
& =\left|\kappa+\frac{a}{s^{1 / 2}}(1+i \delta)-\kappa \frac{b(1+i \delta)}{(p-1)^{2}} \frac{y^{2}}{s^{1 / 2}}+i \kappa \frac{b^{2} \delta(p+1)}{2(p-1)^{4}} \frac{y^{4}}{s}+\kappa \frac{b^{3}}{6(p-1)^{6}} \delta(1+p)(\delta-i(2 p-1)) \frac{y^{6}}{s^{3 / 2}}+O\left(\frac{y^{8}}{s^{2}}\right)\right|^{p-1} \\
& =\left[\left(\kappa+\frac{a}{s^{1 / 2}}-\frac{\kappa b}{(p-1)^{2}} \frac{y^{2}}{s^{1 / 2}}+\kappa \frac{b^{3} \delta^{2}(1+p)}{6(p-1)^{6}} \frac{y^{6}}{s^{3 / 2}}+O\left(\frac{y^{8}}{s^{2}}\right)\right)^{2}+\delta^{2}\left(\frac{a}{s^{1 / 2}}-\frac{\kappa b}{(p-1)^{2}} \frac{y^{2}}{s^{1 / 2}}+\kappa \frac{b^{2}(p+1)}{2(p-1)^{4}} \frac{y^{4}}{s}+O\left(\frac{y^{6}}{s^{3 / 2}}\right)^{2}\right]^{\frac{p-1}{2}}\right. \\
& =\left(\left(\kappa+\frac{a}{s^{1 / 2}}\right)^{2}+\frac{\delta^{2} a^{2}}{s}-2\left(\kappa+\frac{a}{s^{1 / 2}}\right) \frac{\kappa b}{(p-1)^{2}} \frac{y^{2}}{s^{1 / 2}}-2 \frac{\delta^{2} \kappa a b}{(p-1)^{2}} \frac{y^{2}}{s}+\frac{\kappa^{2} b^{2}(p+1)}{(p-1)^{4}} \frac{y^{4}}{s}\right. \\
& \left.+\kappa \frac{a b^{2} \delta^{2}(p+1)}{(p-1)^{4}} \frac{y^{4}}{s^{3 / 2}}+\kappa^{2} \frac{b^{3} \delta^{2}(p+1)}{(p-1)^{6}} \frac{1-3}{3} \frac{y^{6}}{s^{3 / 2}}+O\left(\frac{y^{6}}{s^{2}}\right)+O\left(\frac{y^{8}}{s^{2}}\right)\right)^{\frac{p-1}{2}} \\
& =\left(\kappa^{2}+\frac{2 \kappa a}{s^{1 / 2}}+\frac{a^{2}(p+1)}{s}-2 \frac{b \kappa^{2}}{(p-1)^{2}} \frac{y^{2}}{s^{1 / 2}}-2 \frac{\kappa a b(p+1)}{(p-1)^{2}} \frac{y^{2}}{s}+\frac{\kappa^{2} b^{2}(p+1)}{(p-1)^{4}} \frac{y^{4}}{s}+\kappa \frac{a b^{2} p(p+1)}{(p-1)^{4}} \frac{y^{4}}{s^{3 / 2}}-\frac{2}{3} \kappa^{2} \frac{b^{3} p(p+1)}{(p-1)^{6}} \frac{y^{6}}{s^{3 / 2}}\right. \\
& \left.+O\left(\frac{y^{6}}{s^{2}}\right)+O\left(\frac{y^{8}}{s^{2}}\right)\right)^{\frac{p-1}{2}} \text {. } \\
& =\kappa^{p-1}\left(1+\frac{2 a}{\kappa s^{1 / 2}}+\frac{a^{2}(p+1)}{\kappa^{2} s}\right)^{\frac{p-1}{2}}\left(1-2 \frac{b}{(p-1)^{2}} \frac{1}{1+\frac{2 a}{\kappa s^{1 / 2}}+\frac{a^{2}(p+1)}{\kappa^{2} s}}\left[\frac{y^{2}}{s^{1 / 2}}+\frac{a(p+1)}{\kappa} \frac{y^{2}}{s}-\frac{b(p+1)}{2(p-1)^{2}} \frac{y^{4}}{s}\right.\right. \\
& \left.\left.-\frac{a b p(p+1)}{2 \kappa(p-1)^{2}} \frac{y^{4}}{s^{3 / 2}}+\frac{1}{3} \frac{b^{2} p(p+1)}{(p-1)^{4}} \frac{y^{6}}{s^{3 / 2}}+O\left(\frac{y^{6}}{s^{2}}\right)+O\left(\frac{y^{8}}{s^{2}}\right)\right]\right)^{\frac{p-1}{2}} \\
& =\kappa^{p-1}\left(1+\frac{2 a}{\kappa s^{1 / 2}}+\frac{a^{2}(p+1)}{\kappa^{2} s}\right)^{\frac{p-1}{2}}\left(1-2 \frac{b}{(p-1)^{2}}\left[1-\frac{2 a}{\kappa s^{1 / 2}}-\frac{a^{2}(p+1)}{\kappa^{2} s}+\frac{4 a^{2}}{\kappa^{2} s}+O\left(\frac{1}{s^{3 / 2}}\right)\right]\right. \\
& \left.*\left[\frac{y^{2}}{s^{1 / 2}}+\frac{a(p+1)}{\kappa} \frac{y^{2}}{s}-\frac{b(p+1)}{2(p-1)^{2}} \frac{y^{4}}{s}-\frac{a b p(p+1)}{2 \kappa(p-1)^{2}} \frac{y^{4}}{s^{3 / 2}}+\frac{1}{3} \frac{b^{2} p(p+1)}{(p-1)^{4}} \frac{y^{6}}{s^{3 / 2}}+O\left(\frac{y^{6}}{s^{2}}\right)+O\left(\frac{y^{8}}{s^{2}}\right)\right]\right)^{\frac{p-1}{2}} \\
& =\kappa^{p-1}\left(1+\frac{2 a}{\kappa s^{1 / 2}}+\frac{a^{2}(p+1)}{\kappa^{2} s}\right)^{\frac{p-1}{2}}\left(1-2 \frac{b}{(p-1)^{2}}\left[1-\frac{2 a}{\kappa s^{1 / 2}}-\frac{a^{2}(p-3)}{\kappa^{2} s}+O\left(\frac{1}{s^{3 / 2}}\right)\right]\right. \\
& \left.*\left[\frac{y^{2}}{s^{1 / 2}}+\frac{a(p+1)}{\kappa} \frac{y^{2}}{s}-\frac{b(p+1)}{2(p-1)^{2}} \frac{y^{4}}{s}-\frac{a b p(p+1)}{2 \kappa(p-1)^{2}} \frac{y^{4}}{s^{3 / 2}}+\frac{1}{3} \frac{b^{2} p(p+1)}{(p-1)^{4}} \frac{y^{6}}{s^{3 / 2}}+O\left(\frac{y^{6}}{s^{2}}\right)+O\left(\frac{y^{8}}{s^{2}}\right)\right]\right)^{\frac{p-1}{2}} \\
& =\kappa^{p-1}\left(1+\frac{2 a}{\kappa s^{1 / 2}}+\frac{a^{2}(p+1)}{\kappa^{2} s}\right)^{\frac{p-1}{2}}\left(1-2 \frac{b}{(p-1)^{2}} \frac{y^{2}}{s^{1 / 2}}-2 \frac{a b}{\kappa(p-1)} \frac{y^{2}}{s}+\frac{b^{2}(p+1)}{(p-1)^{4}} \frac{y^{4}}{s}\right. \\
& \left.+2 \frac{a^{2} b(3 p-1)}{\kappa^{2}(p-1)^{2}} \frac{y^{2}}{s^{3 / 2}}+\frac{a b^{2}(p+1)(p-2)}{\kappa(p-1)^{4}} \frac{y^{4}}{s^{3 / 2}}-\frac{2}{3} \frac{b^{3} p(p+1)}{(p-1)^{6}} \frac{y^{6}}{s^{3 / 2}}+O\left(\frac{y^{6}}{s^{2}}\right)+O\left(\frac{y^{8}}{s^{2}}\right)\right)^{\frac{p-1}{2}} \\
& =\kappa^{p-1}\left(1+\frac{(p-1) a}{\kappa s^{1 / 2}}+\frac{a^{2}\left(p^{2}-1\right)}{2 \kappa^{2} s}+\frac{(p-1)(p-3) a^{2}}{2 \kappa^{2} s}++\frac{a^{3}}{2 \kappa^{3}} \frac{\left(p^{2}-1\right)(p-3)}{s^{3 / 2}}+\frac{a^{3}}{6 \kappa^{3}} \frac{(p-1)(p-3)(p-5)}{s^{3 / 2}}+O\left(\frac{1}{s^{2}}\right)\right) \\
& *\left(1-\frac{b}{(p-1)} \frac{y^{2}}{s^{1 / 2}}-\frac{a b}{\kappa} \frac{y^{2}}{s}+\frac{b^{2}(p+1)}{2(p-1)^{3}} \frac{y^{4}}{s}+\frac{(p-3) b^{2}}{2(p-1)^{3}} \frac{y^{4}}{s}\right. \\
& +\frac{a^{2} b(3 p-1)}{\kappa^{2}(p-1)} \frac{y^{2}}{s^{3 / 2}}+\frac{a b^{2}(p+1)(p-2)}{2 \kappa(p-1)^{3}} \frac{y^{4}}{s^{3 / 2}}+\frac{a b^{2}(p-1)(p-3)}{\kappa(p-1)^{3}} \frac{y^{4}}{s^{3 / 2}} \\
& \left.-\frac{b^{3}}{(p-1)^{5}}\left[\frac{1}{3} p(p+1)+\frac{1}{2}(p+1)(p-3)+\frac{1}{6}(p-3)(p-5)\right] \frac{y^{6}}{s^{3 / 2}}+O\left(\frac{y^{6}}{s^{2}}\right)+O\left(\frac{y^{8}}{s^{2}}\right)\right) \\
& =\kappa^{p-1}\left(1+\frac{(p-1) a}{\kappa s^{1 / 2}}+\frac{a^{2}(p-1)^{2}}{\kappa^{2} s}+\frac{a^{3}}{3 \kappa^{3} s^{3 / 2}}(p-1)(p-3)(2 p-1)+O\left(\frac{1}{s^{2}}\right)\right) \\
& *\left(1-\frac{b}{(p-1)} \frac{y^{2}}{s^{1 / 2}}-\frac{a b}{\kappa} \frac{y^{2}}{s}+\frac{b^{2}}{(p-1)^{2}} \frac{y^{4}}{s}\right. \\
& +\frac{a^{2} b(3 p-1)}{\kappa^{2}(p-1)} \frac{y^{2}}{s^{3 / 2}} \\
& +\frac{a b^{2}}{2 \kappa(p-1)^{3}}[(p+1)(p-2)+2(p-1)(p-3)] \frac{y^{4}}{s^{3 / 2}} \\
& \left.-\frac{b^{3}}{(p-1)^{3}} \frac{y^{6}}{s^{3 / 2}}+O\left(\frac{y^{6}}{s^{2}}\right)+O\left(\frac{y^{8}}{s^{2}}\right)\right) \text {, } \\
& =\kappa^{p-1}\left(1+\frac{(p-1) a}{\kappa s^{1 / 2}}+\frac{a^{2}(p-1)^{2}}{\kappa^{2} s}-\frac{b}{(p-1)} \frac{y^{2}}{s^{1 / 2}}+\frac{a^{3}}{3 \kappa^{3} s^{3 / 2}}(p-1)(p-3)(2 p-1)\right.
\end{aligned}
$$

Now we can write 


$$
\begin{aligned}
& F\left(\varphi_{0}(z)+\frac{a}{s^{1 / 2}}(1+i \delta)\right) \\
& =(1+i \delta) \kappa^{p-1}\left(1+\frac{(p-1) a}{\kappa s^{1 / 2}}+\frac{a^{2}(p-1)^{2}}{\kappa^{2} s}-\frac{b}{(p-1)} \frac{y^{2}}{s^{1 / 2}}+\frac{a^{3}}{3 \kappa^{3} s^{3 / 2}}(p-1)(p-3)(2 p-1)\right. \\
& -\frac{2 a b}{\kappa} \frac{y^{2}}{s}+\frac{a^{2} b(p-3)(1-2 p)}{\kappa^{2}(p-1)} \frac{y^{2}}{s^{3 / 2}} \\
& +\frac{b^{2}}{(p-1)^{2}} \frac{y^{4}}{s}+\frac{a b^{2}}{2 \kappa(p-1)^{3}}(p-2)(5 p-3) \frac{y^{4}}{s^{3 / 2}} \\
& \left.-\frac{b^{3}}{6(p-1)^{3}} \frac{y^{6}}{s^{3 / 2}}+O\left(\frac{1}{s^{2}}\right)+O\left(\frac{y^{8}}{s^{2}}\right)\right) . \\
& *\left[\kappa+\frac{a(1+i \delta)}{s^{1 / 2}}-\frac{\kappa b(1+i \delta)}{(p-1)^{2}} \frac{y^{2}}{s^{1 / 2}}+i \kappa \frac{b^{2} \delta(p+1)}{2(p-1)^{4}} \frac{y^{4}}{s}-\kappa \frac{b^{3}}{6(p-1)^{6}} \delta(1+p)(\delta+i(1-2 p)) \frac{y^{6}}{s^{3 / 2}}+O\left(\frac{y^{8}}{s^{2}}\right)\right] .
\end{aligned}
$$

- $F\left(\varphi_{0}(z)\right)$

$$
\begin{aligned}
& F\left(\varphi_{0}(z)\right)= \\
& (1+i \delta) \kappa^{p}\left|1+\frac{b}{p-1} \frac{y^{2}}{s^{1 / 2}}\right|^{-1}\left(1+\frac{b}{p-1} \frac{y^{2}}{s^{1 / 2}}\right)^{-\frac{1+i \delta}{p-1}} \\
& =(1+i \delta) \kappa^{p}\left(1-\frac{b}{p-1} \frac{y^{2}}{s^{1 / 2}}+\frac{b^{2}}{(p-1)^{2}} \frac{y^{4}}{s}-\frac{b^{3}}{(p-1)^{3}} \frac{y^{6}}{s^{3 / 2}}+O\left(\frac{y^{8}}{s^{2}}\right)\right) \\
& *\left\{1-\frac{b(1+i \delta)}{(p-1)^{2}} \frac{y^{2}}{s^{1 / 2}}+i \frac{b^{2} \delta(p+1)}{2(p-1)^{4}} \frac{y^{4}}{s}-\frac{b^{3}}{6(p-1)^{6}} \delta(1+p)(\delta+i(1-2 p)) \frac{y^{6}}{s^{3 / 2}}+O\left(\frac{y^{8}}{s^{2}}\right)\right\} \\
& =(1+i \delta) \kappa^{p}\left(1-\frac{b}{p-1} \frac{p+i \delta}{p-1} \frac{y^{2}}{s^{1 / 2}}+\left\{i \frac{b^{2} \delta(p+1)}{2(p-1)^{4}}+\frac{b^{2}}{(p-1)^{2}}+\frac{b^{2}(1+i \delta)}{(p-1)^{3}}\right\} \frac{y^{4}}{s}\right. \\
& \left.-\left\{\frac{b^{3}}{(p-1)^{3}}+\frac{b^{3}}{6(p-1)^{6}} \delta(1+p)(\delta+i(1-2 p))+i \frac{b^{3} \delta(p+1)}{2(p-1)^{5}}+\frac{b^{3}}{(p-1)^{4}}(1+i \delta)\right\} \frac{y^{6}}{s^{3 / 2}}+O\left(\frac{y^{8}}{s^{2}}\right)\right) .
\end{aligned}
$$

- Finally the term $\varphi_{0}(z)+\frac{a}{s^{1 / 2}}(1+i \delta)$ :

$$
\varphi_{0}(z)+\frac{a}{s^{1 / 2}}(1+i \delta)=\kappa+\frac{a}{\sqrt{s}}(1+i \delta)-\frac{\kappa b}{(p-1)^{2}}(1+i \delta) \frac{y^{2}}{s^{1 / 2}}+i \kappa \frac{b^{2} \delta(p+1)}{2(p-1)^{4}} \frac{y^{4}}{s}+O\left(\frac{y^{6}}{s^{3 / 2}}\right)
$$

Now, let us see the term of order $\frac{1}{s}$ in the function $R^{*}$, by expansions above, we have

$$
\begin{aligned}
& (1+i \delta) \frac{a^{2}}{\kappa}(p-1+1+i \delta)-i \mu \kappa, \\
& =\frac{a^{2}}{\kappa}(1+i \delta)(p+i \delta)-i \mu \kappa, \\
& =\frac{a^{2}}{\kappa}\left(p-\delta^{2}+i \delta(p+1)\right)-i \mu \kappa \text { we recall that we are in the critical case }, p=\delta^{2} \\
& =i \frac{a^{2}}{\kappa} \delta(1+p)-i \mu \kappa
\end{aligned}
$$

Now, let us see the term of order $\frac{1}{s^{1 / 2}}$ in the function $R^{*}$

$$
\begin{aligned}
& (1+i \delta)\left\{-2 \frac{\kappa b}{(p-1)^{2}}-(1+i \delta) \frac{a}{(p-1)}+a+(1+i \delta) \frac{a}{(p-1)}\right\} \\
& =(1+i \delta)\left\{-2 \frac{\kappa b}{(p-1)^{2}}+a\right\}=0,\left(\text { we recall } b=\frac{(p-1)^{2}}{8 \sqrt{p(p+1)}}, \quad a=\frac{\kappa}{4 \sqrt{p(p+1)}}\right)
\end{aligned}
$$

Now, let us see the term of order $\frac{y^{2}}{s^{1 / 2}}$ in the function $R^{*}$, by expansions above, we have

$$
-2 i \frac{b \kappa}{(p-1)^{2}}(p+1) \delta+2 i \frac{b \kappa}{(p-1)^{2}}(p+1) \delta=0
$$


Now, let us see the term of order $\frac{y^{2}}{s}$ in the function $R^{*}$, by expansions above, we have

$$
i 6 \kappa \frac{\delta b^{2}(p+1)}{(p-1)^{4}}-i \frac{2 a b \delta(p+1)}{(p-1)^{2}}
$$

The term of order $\frac{y^{4}}{s}$ is equal to zero.

Now, let us see the term of order $\frac{1}{s^{3 / 2}}$ in the function $R^{*}$, by expansions above, we have

$$
a\left(\frac{1}{2}+\mu \delta\right)+\frac{a^{3}}{3 \kappa^{2}}(p-3)(2 p-1)+i\left[\frac{\delta a^{3}}{3 \kappa^{2}}(p-3)(2 p-1)-\mu a\right] .
$$

The term of order $\frac{y^{2}}{s^{3 / 2}}$ in the function $R^{*}$,

$\kappa \frac{b}{(p-1)^{2}}\left(-\frac{1}{2}-\mu \delta\right)+\frac{a^{2} b}{\kappa(p-1)^{2}} p(p+1)+i\left[\kappa \frac{b}{(p-1)^{2}}\left(-\frac{\delta}{2}+\mu\right)+\frac{a^{2} b}{\kappa(p-1)^{2}} \delta\left(-2 p^{2}+p+3\right)\right]$

The term of order $\frac{y^{4}}{s^{3 / 2}}$ in the function $R^{*}$,

$$
\kappa \frac{b^{3}}{(p-1)^{6}} 5 p(p+1)-\frac{a b^{2}}{(p-1)^{4}} p(p+1)+i \delta\left[\kappa \frac{b^{3}}{(p-1)^{6}} 5(1-2 p)+\frac{a b^{2}}{(p-1)^{4}}\left(5 p^{2}-5\right)\right]
$$

The term of order $\frac{y^{6}}{s^{3 / 2}}$ in the function $R^{*}$, is equal to zero.

\section{Taylor expansion of $B$}

Let us recall from (43) that:

$$
B(q, y, s)=(1+i \delta)\left(|\varphi+q|^{p-1}(\varphi+q)-|\varphi|^{p-1} \varphi-|\varphi|^{p-1} q-\frac{p-1}{2}|\varphi|^{p-3} \varphi(\varphi \bar{q}+\bar{\varphi} q)\right)
$$

Next, we compute the taylor expansion of $F(q)=|\varphi+q|^{p-1}=\left(\left(\varphi_{1}+q_{1}\right)^{2}+\left(\varphi_{2}+q_{2}\right)^{2}\right)^{(p-1) / 2}$, then an easy calculation shows

$$
\nabla F(q)=(p-1)|\varphi+q|^{p-3}\left(\varphi_{1}+q_{1}, \varphi_{2}+q_{2}\right)
$$

where $\varphi=\varphi_{1}+i \varphi_{2}$ and $q=q_{1}+i q_{2}$, and

$$
H(F)(q)=(p-1)\left(\begin{array}{ll}
|\varphi+q|^{p-3}+(p-3)|\varphi+q|^{p-5}\left(\varphi_{1}+q_{1}\right)^{2} & (p-3)|\varphi+q|^{p-5}\left(\varphi_{1}+q_{1}\right)\left(\varphi_{2}+q_{2}\right) \\
(p-3)|\varphi+q|^{p-5}\left(\varphi_{1}+q_{1}\right)\left(\varphi_{2}+q_{2}\right) & |\varphi+q|^{p-3}+(p-3)|\varphi+q|^{p-5}\left(\varphi_{2}+q_{2}\right)^{2}
\end{array}\right)
$$

Then

$$
\begin{gathered}
\nabla F(0)=(p-1)|\varphi|^{p-3}\left(\varphi_{1}, \varphi_{2}\right) \\
H(F)(0)=(p-1)\left(\begin{array}{ll}
|\varphi|^{p-3}+(p-3)|\varphi|^{p-5} \varphi_{1}^{2} & (p-3)|\varphi|^{p-5} \varphi_{1} \varphi_{2} \\
\frac{(p-3)}{2}|\varphi|^{p-5} \varphi_{1} \varphi_{2} & |\varphi|^{p-3}+(p-3)|\varphi|^{p-5} \varphi_{2}^{2} .
\end{array}\right)
\end{gathered}
$$

And we obtain 


$$
\begin{aligned}
B(q, y, s)= & (1+i \delta)\left\{(p-1)|\varphi|^{p-3}\left(\varphi_{1} q_{1}+\varphi_{2} q_{2}\right) q\right. \\
& +\frac{(p-1)}{2}\left[\left(|\varphi|^{p-3}+(p-3)|\varphi|^{p-5} \varphi_{1}^{2}\right) \varphi q_{1}^{2}+\left(|\varphi|^{p-3}+(p-3)|\varphi|^{p-5} \varphi_{2}^{2}\right) \varphi q_{2}^{2}\right. \\
& \left.\left.\left.+2(p-3)|\varphi|^{p-5} \varphi \varphi_{1} \varphi_{2} q_{1} q_{2}\right)\right]\right\}+O\left(q^{3}\right)
\end{aligned}
$$

Using the fact that $\varphi_{1}=\kappa+O\left(\frac{y^{2}}{\sqrt{s}}\right), \varphi_{2}=O\left(\frac{y^{2}}{\sqrt{s}}\right)$ and by the decomposition of $q$ given by (53), we can deduce that the contribution of $\tilde{q}_{2}^{2}$ is given by the following

$$
\begin{aligned}
& (1+i \delta)\left\{(p-1) \kappa^{p-2}(1+i \delta)+\frac{p-1}{2}\left(1+(p-3)+\delta^{2}\right)\right\} \tilde{q}_{2}^{2} h_{2}^{2} \\
& =(p-1) \kappa^{p-2}\left[(1+i \delta)^{2}+(1+i \delta)(p-1)\right] \tilde{q}_{2}^{2} h_{2}^{2}
\end{aligned}
$$

We note that

$$
\begin{gathered}
\tilde{P}_{2}\left((1+i \delta)^{2} h_{2}^{2}\right)=8\left(1-\delta^{2}\right)=8(1-p) . \\
\tilde{P}_{2}\left((1+i \delta) h_{2}^{2}\right)=8 .
\end{gathered}
$$

Then, we conclude that the contribution of $\tilde{q}_{2}^{2}$ in $\tilde{P}_{2}(B)$ is zero. 


\section{Funding:}

Hatem Zaag is supported by the ERC Advanced Grant no. 291214, BLOWDISOL, and by ANR project no. ANR-13-BS01-0010-03, ANAÉ.

\section{Conflict of Interest:}

The authors declare that they have no conflict of interest. 


\section{References}

[AK02] I.S. Aranson and L. Kramer. The world of the complex Ginzburg-Landau equation. Rev. Modern Phys., 74(1):99-143, 2002.

[BK94] J. Bricmont and A. Kupiainen. Universality in blow-up for nonlinear heat equations. Nonlinearity, 7(2):539-575, 1994.

[Caz03] T. Cazenave. Semilinear Schrödinger equations, volume 10 of Courant Lecture Notes in Mathematics. New York University, Courant Institute of Mathematical Sciences, New York; American Mathematical Society, Providence, RI, 2003.

[CZ13] R. Côte and H. Zaag. Construction of a multi-soliton blow-up solution to the semilinear wave equation in one space dimension. Comm. Pure Appl. Math., 66(10):1541-1581, 2013.

[EZ11] M. A. Ebde and H. Zaag. Construction and stability of a blow up solution for a nonlinear heat equation with a gradient term. S $\overrightarrow{\mathrm{e}} M A$ J., 55:5-21, 2011.

[GK89] Y. Giga and R. V. Kohn. Nondegeneracy of blowup for semilinear heat equations. Comm. Pure Appl. Math., 42(6):845-884, 1989.

[GV96] J. Ginibre and G. Velo. The Cauchy problem in local spaces for the complex Ginzburg-Landau equation. Differential equations, asymptotic analysis, and mathematical physics, 100:138-152, 1996.

[GV97] J. Ginibre and G. Velo. The Cauchy problem in local spaces for the complex Ginzburg-Landau equation. II. Contraction methods. Comm. Math. Phys., 187(1):45-79, 1997.

[HS72] L. M. Hocking and K. Stewartson. On the nonlinear response of a marginally unstable plane parallel flow to a two-dimensional disturbance. Proc. Roy. Soc. London Ser. A, 326:289-313, 1972.

[HSSB72] L. M. Hocking, K. Stewartson, Stuart.J.T., and Brown.S.N. A nonlinear instability in plane parallel flow. J. Fluid. Mech, 51:705-735, 1972.

[KBS88] P. Kolodner, D. Bensimon, and M. Surko. Traveling wave convection in an annulus. Phys.Rev.Lett., 60:1723-, 1988.

[KSAL95] P. Kolodner, S. Slimani, N. Aubry, and R. Lima. Characterization of dispersive chaos and related states of binary-fluid convection. Phys. D, 85(1-2):165-224, 1995.

[Mer92] F. Merle. Solution of a nonlinear heat equation with arbitrary given blow-up points. Comm. Pure Appl. Math., 45(3):263-300, 1992.

[MZ97] F. Merle and H. Zaag. Stability of the blow-up profile for equations of the type $u_{t}=\Delta u+|u|^{p-1} u$. Duke Math. J., 86(1):143-195, 1997.

[MZ08] N. Masmoudi and H. Zaag. Blow-up profile for the complex Ginzburg-Landau equation. J. Funct. Anal., 225:1613-1666, 2008. 
[NZ10] N. Nouaili and H. Zaag. A liouville theorem for vector valued semilinear heat equations with no gradient structure and applications to blow-up. Trans. Amer. Math. Soc., 362(7):3391-3434, 2010.

[NZ15a] V.T. Nguyen and H. Zaag. Construction of a stable blow-up solution for a class of strongly perturbed semilinear heat equations. 2015. Submitted.

[NZ15b] N. Nouaili and H. Zaag. Profile for a simultaneously blowing up solution to a complex valued semilinear heat equation. Comm. Partial Differential Equations, (7):1197-1217, 2015.

[PŠ01] P. Plecháč and V. Šverák. On self-similar singular solutions of the complex Ginzburg-Landau equation. Comm. Pure Appl. Math., 54(10):1215-1242, 2001.

[PSKK98] S. Popp, O. Stiller, E. Kuznetsov, and L. Kramer. The cubic complex GinzburgLandau equation for a backward bifurcation. Phys. D, 114(1-2):81-107, 1998.

[Rot08] V. Rottschäfer. Multi-bump, self-similar, blow-up solutions of the GinzburgLandau equation. Phys. D, 237(4):510-539, 2008.

[Rot13] V. Rottschäfer. Asymptotic analysis of a new type of multi-bump, self-similar, blowup solutions of the Ginzburg-Landau equation. European J. Appl. Math., 24(1):103-129, 2013.

[RS13] P. Raphaël and R. Schweyer. Stable blowup dynamics for the 1-corotational energy critical harmonic heat flow. Comm. Pure Appl. Math., 66(3):414-480, 2013.

[RS14] P. Raphaël and R. Schweyer. On the stability of critical chemotactic aggregation. Math. Ann., 359(1-2):267-377, 2014.

[SS71] K. Stewartson and J. T. Stuart. A non-linear instability theory for a wave system in plane Poiseuille flow. J. Fluid Mech., 48:529-545, 1971.

[TZ15] S. Tayachi and H. Zaag. Existence of a stable blow-up profile for the nonlinear heat equation with a critical power nonlinear gradient term. 2015. arXiv preprint:1506.08306.

[Vel92] J. J. L. Velázquez. Higher-dimensional blow up for semilinear parabolic equations. Comm. Partial Differential Equations, 17(9-10):1567-1596, 1992.

[Vel93a] J. J. L. Velázquez. Classification of singularities for blowing up solutions in higher dimensions. Trans. Amer. Math. Soc., 338(1):441-464, 1993.

[Vel93b] J. J. L. Velázquez. Estimates on the $(n-1)$-dimensional Hausdorff measure of the blow-up set for a semilinear heat equation. Indiana Univ. Math. J., 42(2):445-476, 1993.

[VGH91] J. J. L. Velázquez, V. A. Galaktionov, and M. A. Herrero. The space structure near a blow-up point for semilinear heat equations: a formal approach. $Z h$. Vychisl. Mat. i Mat. Fiz., 31(no. 3), 1991. 
[Zaa98] H. Zaag. Blow-up results for vector-valued nonlinear heat equations with no gradient structure. Ann. Inst. H. Poincaré Anal. Non Linéaire, 15(5):581-622, 1998.

[Zaa01] H. Zaag. A Liouville theorem and blowup behavior for a vector-valued nonlinear heat equation with no gradient structure. Comm. Pure Appl. Math., 54(1):107$133,2001$.

[Zaa02a] H. Zaag. On the regularity of the blow-up set for semilinear heat equations. Ann. Inst. H. Poincaré Anal. Non Linéaire, 19(5):505-542, 2002.

[Zaa02b] H. Zaag. One-dimensional behavior of singular $N$-dimensional solutions of semilinear heat equations. Comm. Math. Phys., 225(3):523-549, 2002.

[Zaa02c] H. Zaag. Regularity of the blow-up set and singular behavior for semilinear heat equations. In Mathematics $\&$ mathematics education (Bethlehem, 2000), pages 337-347. World Sci. Publishing, River Edge, NJ, 2002. 